\title{
GEOMETRIC OPTIMIZATION FOR A THERMAL MICROFLUIDIC CHIP
}

by

Meysam Rahmat

September 2007

\author{
Department of Mechanical Engineering \\ McGill University, Montreal, QC, Canada, H3A 2K6
}

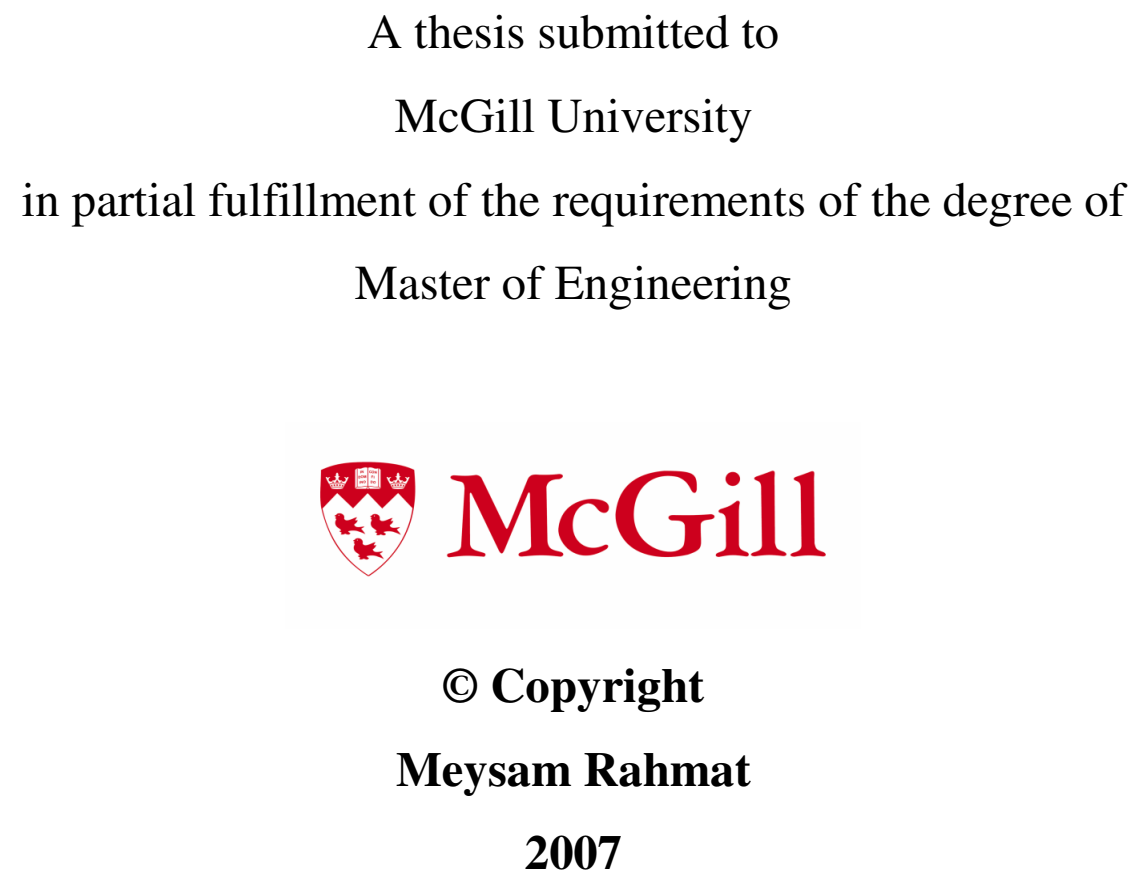


To whom the whole world is waiting for 


\begin{abstract}
During the past two decades, thermal microfluidic chips have significantly been investigated. Due to their high capacity of heat transport, an increasing number of studies on different aspects of thermal microfluidic chips have been conducted. However, a comprehensive investigation on the geometry of microfluidic chips using state of the art finite element software is absent from the literature. In this thesis, geometric parameters of thermal microfluidic chips have been optimized, using finite element software. Consequently, micro and macro phenomena were investigated in different models. The micro modelling approach investigated single microchannels and optimized the microchannel cross-section. Furthermore, two-phase flows in the microchannel were modelled, using finite element software ANSYS CFX. Liquid accumulation in the sharp corners of the microchannel was captured in the model and the phase change phenomenon was observed. The results of the finite element analysis were compared to the literature and a good correlation was observed. The configuration of microchannels in a microfluidic chip was studied through the macro modelling approach. Dimensionless design charts were presented in this section to be employed for all kinds of thermal microfluidic chips with different boundary conditions. Based on the validity of the finite element software, two-phase flows in the optimized three-dimensional network of microchannels were modelled. The results showed the circulation of the two phases in the microchannels and demonstrated the proper operation of the thermal microfluidic chip.
\end{abstract}




\section{Résumé}

Au cours des deux dernières décades, les puces thermiques micro-fluidiques ont été considérablement examinées. Du fait de leur haute capacité pour le transport de chaleur, de nombreuses études ont été réalisées sur différents aspects de leurs propriétés. Cependant, une étude de la géométrie des puces micro-fluidiques utilisant un logiciel d'analyse par éléments finis est absente de la littérature. Dans cette thèse, des paramètres géométriques des puces thermiques micro-fluidiques ont été optimisés en utilisant un logiciel d'analyse par éléments finis. Ainsi, les phénomènes micro et macro ont été étudiés dans différents modèles. L'approche micro a consisté à étudier les micro-canaux seuls, et à optimiser la géométrie de leur section transverse. De plus, deux phases d'écoulement ont été modélisées en utilisant le logiciel d'élément fini ANSYS CFX. L'accumulation de liquide dans les coins saillants a été saisie par le modèle et le phénomène de changement de phase a pu être également observé. Les résultats de l'analyse par élément finis ont été comparés à ceux trouvés dans la littérature, et une bonne corrélation a été observée. La configuration des micro-canaux dans la puce micro-fluidique a été étudiée par l'approche macro. Des graphes adimensionnels ont été présentés dans cette section afin d'être employés pour toutes sortes de puces ayant différentes conditions aux frontières. En se basant sur la validité du modèle micro, élaboré par élément finis, l'écoulement des deux phases dans un réseau tridimensionnel de micro-canaux avec une géométrie optimisée a été modélisé. Les résultats montrent une circulation des deux phases dans les micro-canaux et démontrent le bon fonctionnement des puces thermiques micro fluidiques. 


\section{Acknowledgement}

I would like to express my sincere gratitude to my thesis supervisor, Professor Pascal Hubert, for his invaluable guidance, continued support and encouragement during the course of this research.

Special thank goes to my colleagues in Ecole Polytechnique de Montreal, Professor Sylvain Martel, Professor Daniel Therriault, and, Ramzi Bey-Oueslati, who significantly helped me during the hours of discussions. Moreover, I acknowledge Romain Nicolas from Ecole Polytechniqie de Paris, for the helpful experimental investigations, during his stay in Montreal.

I also extend my sincere appreciation to Lolei Khoun for her help in translating the abstract to French.

I further wish to thank all professors and students in the Composite Materials and Structures laboratory. The friendly and scientific environment in the group led to a smooth approach to every problem.

Finally, I wish to express my endless gratitude to my lovely family. Without any doubt, their undying supports in every step of my life have significantly encouraged me, and I can never be thankful enough.

The valuable financial supports from the Natural Sciences and Engineering Research Council of Canada and also the McGill Max Stern Scholarship are acknowledged. 


\section{Table of Contents}

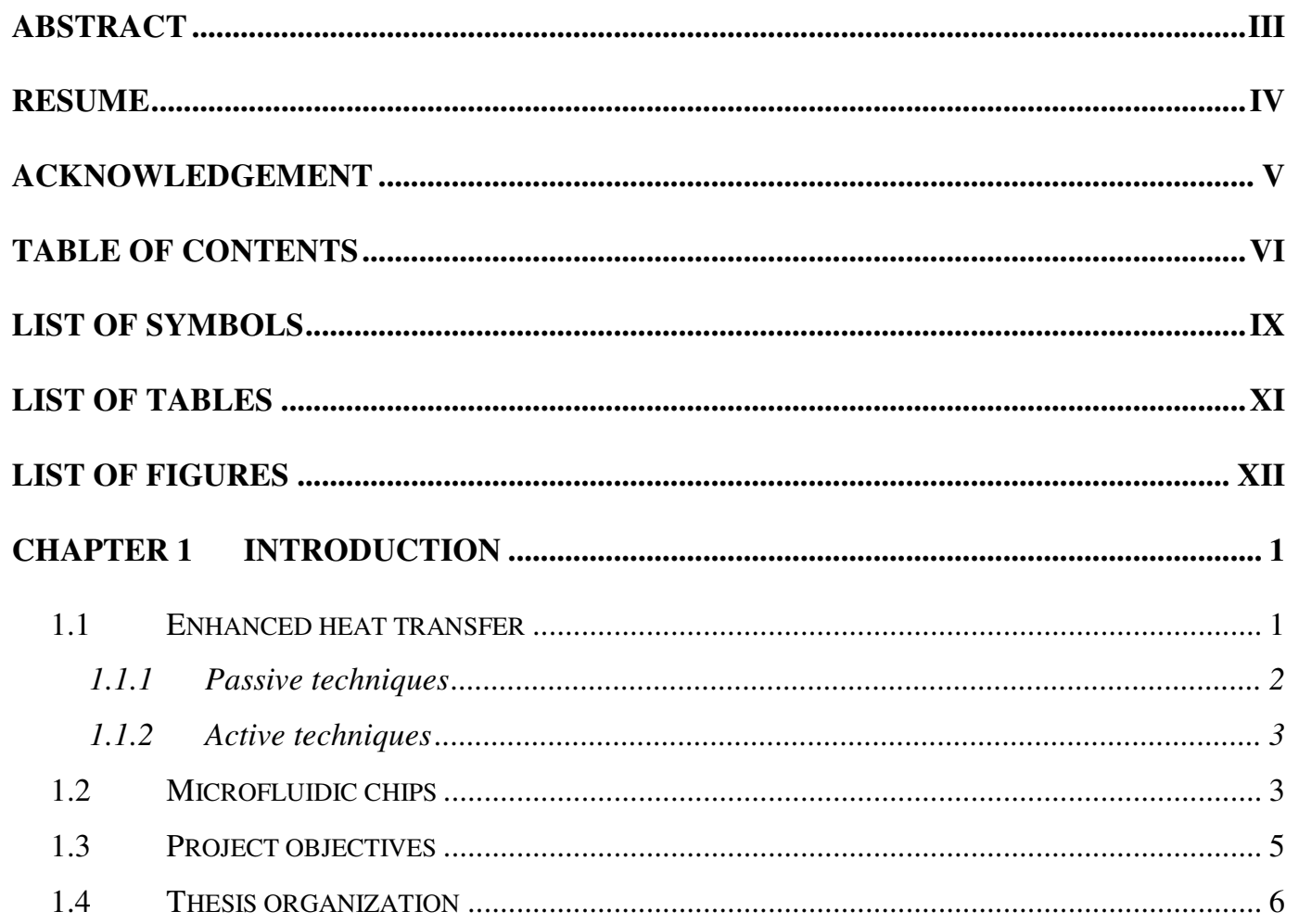

CHAPTER 2 LITERATURE REVIEW ....................................................................................... 7

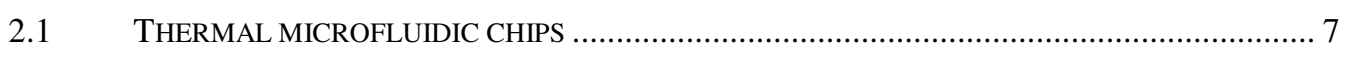

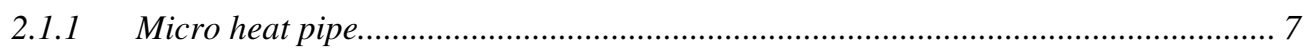

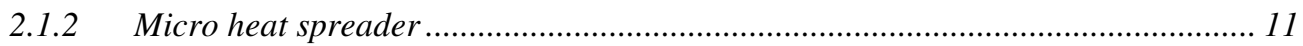

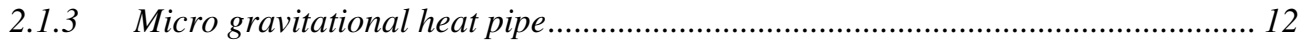

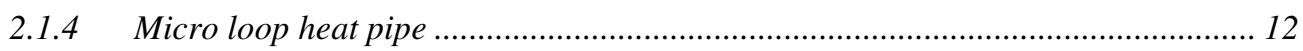

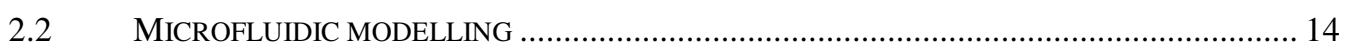

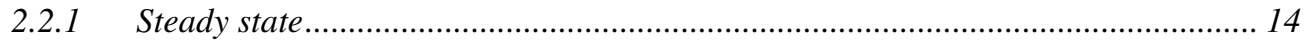

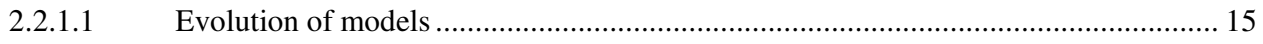

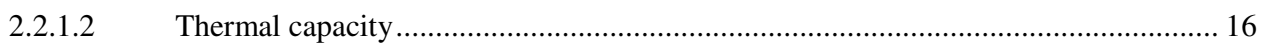

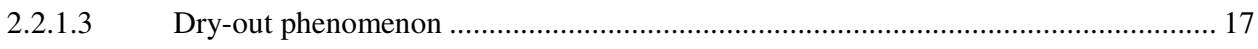

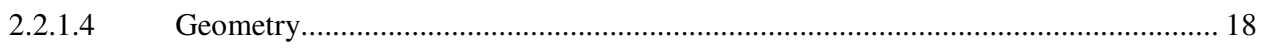

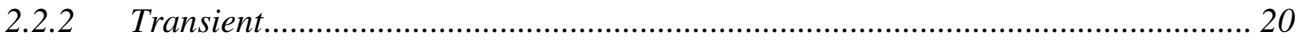

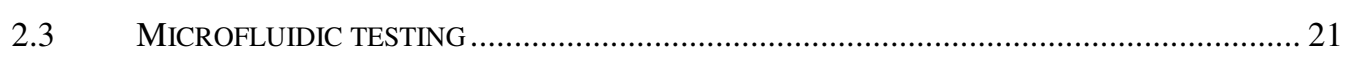

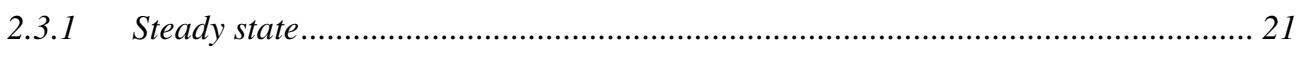

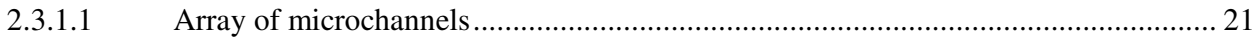

2.3.1.2 Flow observation ........................................................................................ 22

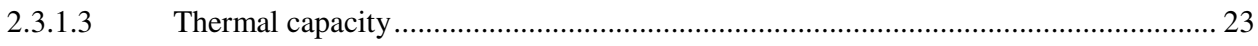




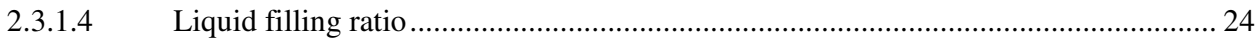

2.3.2 Transient............................................................................................. 25

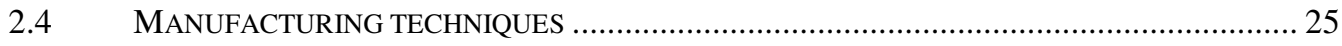

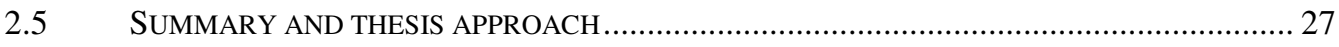

CHAPTER 3 MICRO MODELLING ............................................................................... 31

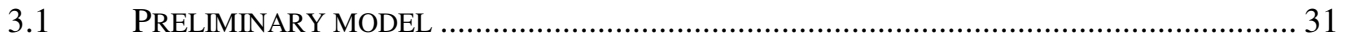

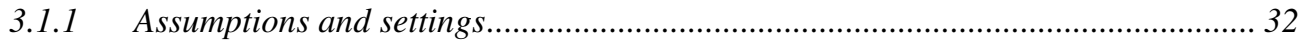

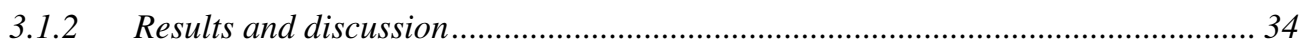

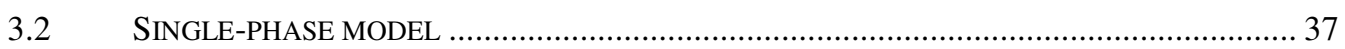

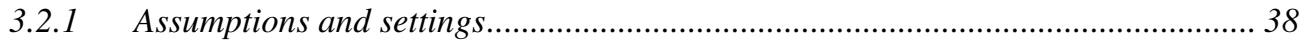

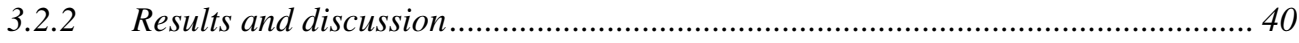

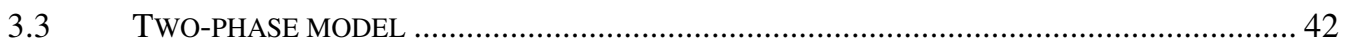

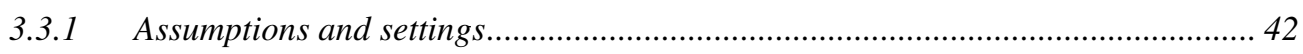

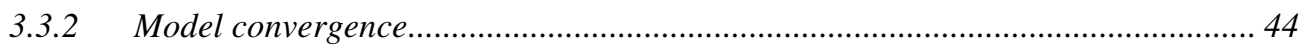

3.3.3 Results and discussion ............................................................................ 46

CHAPTER 4 MACRO MODELLING.................................................................. 63

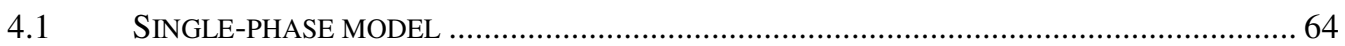

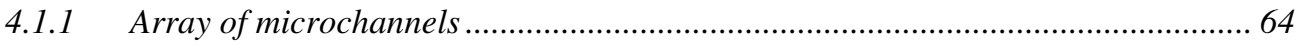

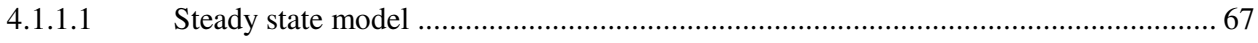

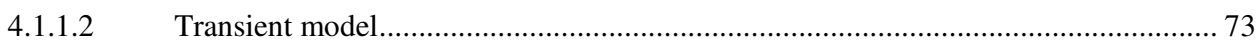

4.1.2 Three-dimensional configuration of microchannels ....................................... 76

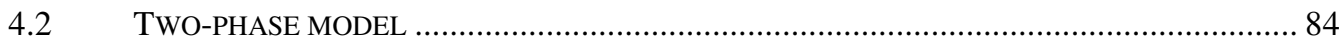

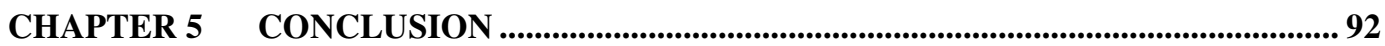

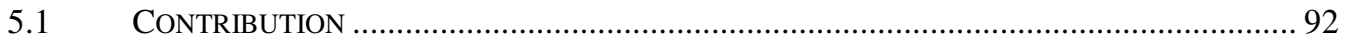

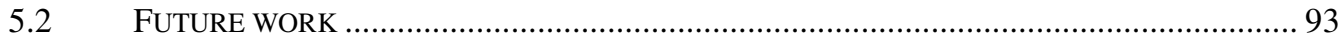

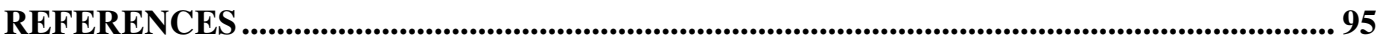

APPENDIX A GOVERNING EQUATIONS............................................................. 99

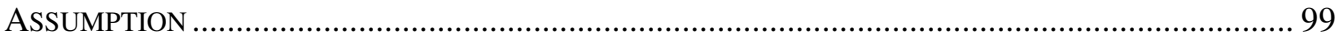

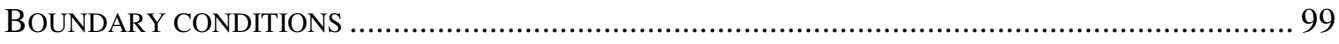

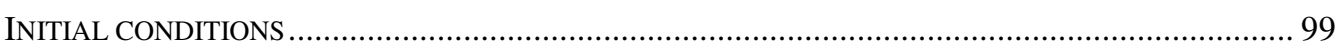

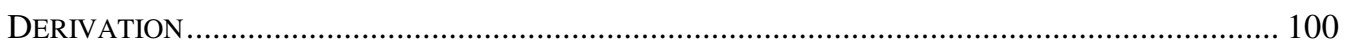

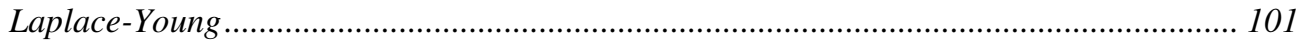

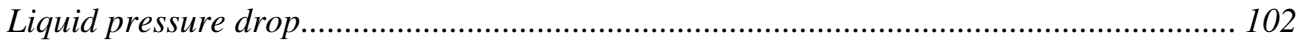

Conservation of momentum for liquid .................................................................. 102

Conservation of momentum for vapour ..................................................................... 103 


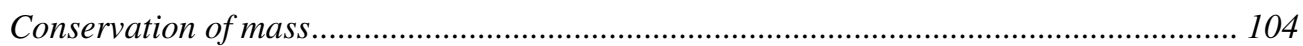

Conservation of energy for liquid ............................................................................... 105

Conservation of energy for vapour ................................................................................ 107 


\section{List of symbols}

\section{Latin Symbols}

$\begin{array}{ll}\text { A } & \text { Area } \\ \text { B } & \text { Body force } \\ \text { C } & \text { Heat capacity } \\ \text { d } & \text { Triangle side; derivation } \\ \text { D } & \text { Material derivation; diameter } \\ \dot{E} & \text { Energy flow rate } \\ \mathrm{f} & \text { Friction factor } \\ \mathrm{F} & \text { Force } \\ \mathrm{g} & \text { Gravitational acceleration } \\ \mathrm{h} & \text { Specific enthalpy; convection heat } \\ \text { transfer coefficient }\end{array}$

$\mathrm{H} \quad$ Height

$\dot{H} \quad$ Rate of enthalpy change

I Interval distance between the microchannels

k Thermal conductivity

K Constant

$K \dot{E} \quad$ Rate of kinetic energy change

1 Length

L Length

$\dot{m} \quad$ Mass flow rate

$\mathrm{p} \quad$ Pressure

$P \dot{E} \quad$ Rate of potential energy change

$\dot{Q} \quad$ Heat flux; heat flow

r Radius of curvature

Re Reynolds number

t Time

T Temperature

u Specific internal energy

U Internal energy

v Velocity

V Velocity
W Width

$\mathrm{x} \quad$ Coordinate axis; length

y Coordinate axis

$\mathrm{z} \quad$ Coordinate axis

\section{Greek Symbols}

$\alpha \quad$ Contact angle

$\beta \quad$ Geometric constant

$\eta \quad$ Half of the wetted length in the side

of triangular channels

$\lambda \quad$ Latent heat of evaporation

$\mu \quad$ Dynamic viscosity

$\rho \quad$ Density

$\sigma \quad$ Surface tension

$\tau \quad$ Shear stress

$\varphi \quad$ Half-channel angle

$\psi \quad$ Tilt angle

\section{Subscripts}

$\begin{array}{ll}\text { adiab } & \text { Adiabatic } \\ \text { avg } & \text { Average } \\ \text { cond } & \text { Condenser } \\ \text { eff } & \text { Effective } \\ \text { evap } & \text { Evaporator } \\ \text { h } & \text { Hydraulic } \\ \text { i } & \text { Interface } \\ \text { in } & \text { In } \\ \text { l } & \text { Liquid } \\ \text { m } & \text { Microchannel } \\ \text { out } & \text { Out } \\ \text { p } & \text { Phase change; constant pressure } \\ \text { ref } & \text { Reference } \\ \text { sat } & \text { Saturation } \\ \text { v } & \text { Vapour } \\ \text { vis } & \text { Viscosity }\end{array}$


$\infty \quad$ Infinity

z Z-direction 


\section{List of Tables}

TABLE 3-1: EFFECTIVE MATERIAL PROPERTIES FOR THE MICROCHANNEL......................................... 33

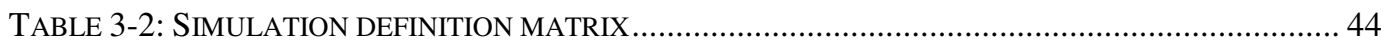

TABLE 4-1: MATERIAL PROPERTIES FOR THE EPOXY AND THE MICROCHANNELS. .............................. 65

TABLE 4-2: DIFFERENT MODEL SETUP FOR TWO-PHASE FLOW IN THREE-DIMENSIONAL GEOMETRY 86 


\section{List of Figures}

FIGURE 1-1: ENHANCED SURFACES: A) OFFSET STRIP FINS USED IN PLATE-FIN HEAT EXCHANGERS, B) SEGMENTED FINS FOR CIRCULAR TUBES, C) INTEGRAL ALUMINIUM STRIP-FINNED TUBE [1].... 3

FIGURE 1-2: EFFECTIVE THERMAL CONDUCTIVITY [2], [4] ...................................................... 5

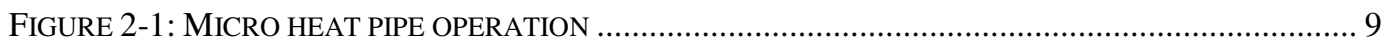

FIGURE 2-2: MiCRO HEAT SPREADER, A) OPERATION, B) VAPOUR FLOW LAYER, C) MID-LAYER, D)

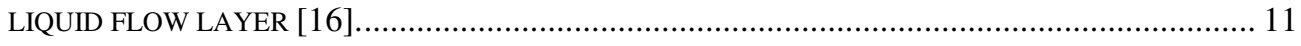

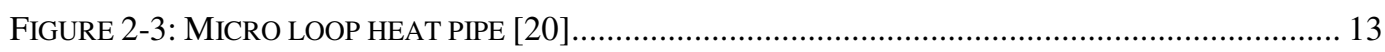

FiguRE 2-4: A) THE ONE-DIMENSIONAL INTERFACE ADDRESSED By LONGTIN ET AL. [24]. B) THE TWO-DIMENSIONAL INTERFACES BETWEEN LIQUID AND VAPOUR ADDRESSED BY STEPHAN

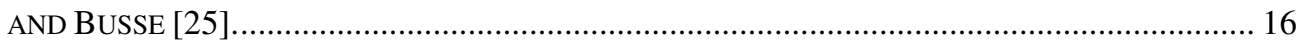

FigURE 2-5: THE FLOW PATTERN AT A) EVAPORATOR, B) ADIABATIC SECTION, AND C) CONDENSER

FigURE 2-6: DIRECT-WRITE TECHNIQUE, A) INK DEPOSITION, B) RESIN INFILTRATION, C) RESIN SOLIDIFICATION, D) INK REMOVAL [56] 27

FigURE 2-7: A) INVESTIGATION METHOD, B) WORKING FLUID, C) SUBSTRATE, D) CHANNEL CROSSSECTION

FiguRE 2-8: MicRo HEAT PIPE MODELLING FLOWCHART FOR MICRO AND MACRO SIMULATIONS .. 30

FIGURE 3-1: MODEL OF A SINGLE MICROCHANNEL 33

FIGURE 3-2: ISO-SURFACES OF HEAT FLUX (W/M²) IN THE REORIENTATION REGION (SEE FIGURE 3-1), A) IN THE X-DIRECTION, B) IN THE Y-DIRECTION. C) THE LENGTH OF THE REORIENTATION ZONE WITH THE X-DIRECTION HEAT FLUX (ON THE TOP) AND Y-DIRECTION HEAT FLUX (ON THE ВОТТОМ).

FigURE 3-3: HEAT FLUX FROM BOTTOM $(\mathrm{Y}=0 \mu \mathrm{M})$, TOP $(\mathrm{Y}=200 \mu \mathrm{M})$, AND MIDDLE $(\mathrm{Y}=100 \mu \mathrm{M})$ OF

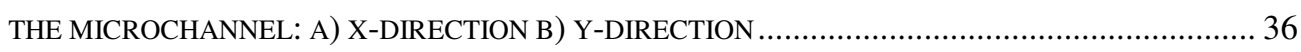

FIGURE 3-4: MODELLING SECTION, MICROCHANNEL CROSS-SECTION GEOMETRIES AND DIMENSIONS. THE VERTICAL LINE ON THE CROSS-SECTION GEOMETRY CORRESPONDS TO THE MODEL PLANE OF SYMMETRY.

FIGURE 3-5: TYPICAL BOUNDARY CONDITIONS APPLIED TO THE MICROCHANNEL; THE TRIANGULAR CROSS-SECTION AND THE CONTOUR PLANE OF FIGURE 3-6 SHOWN (DASHED LINE).

FIGURE 3-6: STEADY STATE RESULTANT VELOCITY (M/S) CONTOURS AT A DISTANCE OF $10 \mu \mathrm{M}$ FROM THE PLANE OF SYMMETRY (SEE FIGURE 3-5), THE LEFT SIDE CORRESPONDS TO THE TOP OF THE MICROCHANNEL FOR A) CIRCULAR, B) TRIANGULAR, AND C) TRAPEZOIDAL CROSS-SECTION. 40

FiguRE 3-7: A) STEADY STATE TEMPERATURE (K) PROFILE AND COOLING LENGTH FOR THE TRIANGULAR CROSS-SECTION ON A PLANE AT A DISTANCE OF 10 MM FROM THE PLANE OF 
SYMMETRY (SEE FIGURE 3-5). B) DIMENSIONLESS COOLING LENGTH FOR DIFFERENT MICROCHANNEL CROSS-SECTIONS

FIGURE 3-8: GEOMETRY AND BOUNDARY CONDITIONS OF THE SYMMETRIC MODEL FOR TWO-PHASE ANALYSIS OF A SINGLE MICRO HEAT PIPE

FIGURE 3-9: SIMULATION CONVERGENCE FOR THE RUN 10, WITH THE RANGE OF DIFFERENCE FOR DIFFERENT RMS'S

FIGURE 3-10: AVERAGE RMS FOR DIFFERENT ANALYSES. THE RED LINE INDICATES THE TARGET RMS OF 1E-4 46

FIGURE 3-11: VAPOUR CONSERVATIVE VOLUME FRACTION FOR A) RUN 11, AND B) RUN 12 ..... 47

FIGURE 3-12: WATER VOLUME FRACTION IN THE EVAPORATOR FOR THE RUN 2.IN THE PLANE OF SYMMETRY.

FIGURE 3-13: WATER/WATER VAPOUR INTERPHASE MASS TRANSFER RATE (KG/M $\left.\mathrm{M}^{3} \mathrm{~S}\right)$ IN THE PLANE OF SYMMETRY A) FOR THE RUN 1, AND B) FOR THE RUN 9. THE TOP FIGURES SHOW THE EVAPORATOR, THE MIDDLE FIGURE DEMONSTRATES THE ADIABATIC SECTION, AND THE BOTTOM ONES INDICATE THE CONDENSER. 49

FIGURE 3-14: LIQUID/GAS INTERFACE IN THE EXPERIMENTAL INVESTIGATIONS [64]..... 50

FIGURE 3-15: A) WATER VAPOUR TEMPERATURE (K) IN THE PLANE OF SYMMETRY FOR THE RUN 2, B) WATER VAPOUR SHEAR STRAIN RATE (1/S) IN THE PLANE OF SYMMETRY FOR THE RUN 1. THE TOP FIGURES SHOW THE EVAPORATOR, THE MIDDLE FIGURES DEMONSTRATE THE ADIABATIC SECTION, AND THE BOTTOM ONES INDICATE THE CONDENSER.

FIGURE 3-16: WATER TEMPERATURE (K) IN THE PLANE OF SYMMETRY FOR THE RUN 1 ............... 53

Figure 3-17: PrESSURE (PA) ALONG THE LINE EE' FOR THE RUN 12 .......................................... 54

FIGURE 3-18: WATER VAPOUR CONSERVATIVE VOLUME FRACTION ALONG THE LINE EE' FOR THE RUN 12

FIGURE 3-19: WATER VAPOUR VERTICAL VELOCITY (M/S) ALONG THE LINE EE' FOR THE RUN 12 . 56 FigurE 3-20: WATER VAPOUR AXIAL VELOCITY (M/S) ALONG THE LINE EE' FOR THE RUN 11 ....... 57

FIGURE 3-21: WATER AXIAL VELOCITY (M/S) ALONG THE LINE FF' FOR THE RUN 12 ................... 58

FIGURE 3-22: WATER/WATER VAPOUR INTERPHASE MASS TRANSFER RATE (KG/M $\left.{ }^{3} \mathrm{~S}\right)$ ALONG THE LINE EE' FOR THE RUN 11 59

FIGURE 3-23: A) WATER VAPOUR CONSERVATIVE VOLUME FRACTION, AND B) WATER VAPOUR AXIAL VELOCITY (M/S) ALONG THE LINE EE' FOR THE RUN 7 60

FIGURE 3-24: DIMENSIONLESS EFFECTIVE LENGTH AS A FUNCTION OF LIQUID FILLING RATIO 61

FigurE 4-1: STEADY STATE TEMPERATURE CONTOUR $\left({ }^{\circ} \mathrm{C}\right)$ FOR ARRAY OF CHANNELS WITH A) 1.07 MM, AND B) 1.2 MM GAP DISTANCE BETWEEN THE CHANNELS 65

FIGURE 4-2: TOTAL HEAT FLUX (W/M²) FOR ARRAY OF CHANNELS WITH A) 1.07 MM, AND B) 1.2 MM GAP DISTANCE BETWEEN THE CHANNELS. 66

FIGURE 4-3: GEOMETRIC PARAMETERS USED IN THE PARAMETRIC MODEL 67 
FIGURE 4-4: TYPICAL BOUNDARY CONDITIONS APPLIED TO THE ADIABATIC SECTION OF MICROCHANNELS ARRAY

FIGURE 4-5: STEADY STATE TEMPERATURE CONTOUR $\left({ }^{\circ} \mathrm{C}\right)$ FOR AN ARRAY OF CHANNELS. EACH COLOR SHOWS A TEMPERATURE RANGE OF $5^{\circ} \mathrm{C}$. 68

FIGURE 4-6: EFFECT OF DIMENSIONLESS INTERVAL DISTANCE $\left(I / W_{T}\right)$ ON THE INTERFACE TEMPERATURE DISTRIBUTION, $H=400 \mu M, W_{T}=400 \mu M$ AND $W_{B}=0$. 70

FIGURE 4-7: EFFECT OF DIMENSIONLESS WIDTH $\left(W_{B} / W_{T}\right)$ ON THE INTERFACE TEMPERATURE DISTRIBUTION, $H=200 \mu M, W_{T}=400 \mu M$ AND $I=1500 \mu M$ 71

FIGURE 4-8: EFFECT OF DIMENSIONLESS HEIGHT $\left(H / W_{T}\right)$ ON THE INTERFACE TEMPERATURE DISTRIBUTION, $I=800 \mu M, W_{T}=400 \mu M$ AND $W_{B}=0 \mu M$

FIGURE 4-9: TRANSIENT RESPONSE OF MICROFLUIDIC CHIP IN DIFFERENT POSITIONS OF TOP SURFACE FOR A) MICROFLUIDIC CHIP MICROCHANNEL AND B) SURROUNDING EPOXY 75

FIGURE 4-10: INTERFACE TEMPERATURE GRADIENT ALONG THE MICROFLUIDIC CHIP LENGTH AT DIFFERENT TIME FOR A) MICROFLUIDIC CHIP MICROCHANNEL AND B) SURROUNDING EPOXY 76

FIGURE 4-11: THE ORDER OF MICROCHANNEL LAYERS FOR A) ONE STEP INFILTRATION (CASE1), AND B) LAYER-BY-LAYER INFILTRATION (CASE 2). 77

FIGURE 4-12: THREE CASES FOR THREE-DIMENSIONAL GEOMETRIC STUDY, A) CASE 1, B) CASE 2, AND C) CASE 3

FIGURE 4-13: INTERCONNECTION BETWEEN THE MICROCHANNELS IN DIFFERENT LAYERS FOR A) THE REAL, AND B) THE IDEAL SITUATION 79

FIGURE 4-14: BOUNDARY CONDITIONS FOR ALL THREE CASES IN THREE-DIMENSIONAL GEOMETRIC STUDY

FIGURE 4-15: TEMPERATURE CONTOURS IN ${ }^{\circ} \mathrm{C}$ (CASE 1) FOR A) $T>59.9^{\circ} \mathrm{C}$, AND B) $T>60^{\circ} \mathrm{C} \ldots \ldots .80$

FIGURE 4-16: TOTAL HEAT FLUX IN W/M², A) FRONT VIEW, B) SIDE VIEW, AND C) TOP VIEW .......... 82

FIGURE 4-17: TEMPERATURE CONTOUR IN ${ }^{\circ} \mathrm{C}$ FOR $T>59.9^{\circ} \mathrm{C}$ FOR A) CASE 1 , B) CASE 2 , AND C) CASE 3; TEMPERATURE CONTOUR ${ }^{\circ} \mathrm{C}$ FOR $T>60^{\circ} \mathrm{C}$ FOR D) CASE 1 , E) CASE 2 , AND F) CASE 3

FIGURE 4-18: MICROCHANNELS GEOMETRY AND DIMENSIONS. THE WHITE LINES DETERMINE THE PLACE OF THE REPRESENTATIVE PLANE.

FIGURE 4-19: MICROCHANNELS MESHING FOR THE TWO-PHASE FLOW IN THREE-DIMENSIONAL GEOMETRY

FIGURE 4-20: MICROCHANNELS BOUNDARY CONDITIONS FOR TWO-PHASE FLOW IN THREEDIMENSIONAL GEOMETRY

FIGURE 4-21: WATER/WATER VAPOUR INTERPHASE MASS TRANSFER RATE (KG/M $\left.{ }^{3} \mathrm{~S}\right)$ IN THE REPRESENTATIVE PLANE FOR RUN 5

FIGURE 4-22: WATER AXIAL VELOCITY (M/S) IN THE REPRESENTATIVE PLANE FOR RUN 1 88

FIGURE 4-23: VAPOUR VOLUME FRACTION IN THE REPRESENTATIVE PLANE FOR RUN 1 89 
FIGURE 4-24: VAPOUR CONSERVATIVE VOLUME FRACTION IN THE REPRESENTATIVE PLANE FOR RUN

1. 90

FigURE 4-25: VAPOUR TEMPERATURE CONTOUR (K) IN THE REPRESENTATIVE PLANE FOR RUN 3.. 91 FigURE 4-26: VAPOUR TEMPERATURE CONTOUR (K) IN THE REPRESENTATIVE PLANE FOR RUN 4.. 91

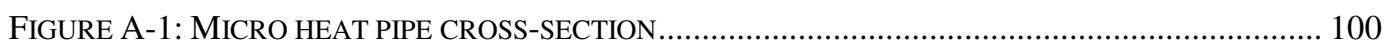

FIGURE A-2: CONSERVATION OF MASS FOR A LIQUID CONTROL VOLUME...................................... 104

FIGURE A-3: CONSERVATION OF ENERGY FOR A LIQUID CONTROL VOLUME .................................. 105

FIGURE A-4: CONSERVATION OF ENERGY FOR A VAPOUR CONTROL VOLUME ................................. 108 


\section{Chapter 1 Introduction}

During the last decades, microelectronic industry has been moving towards the maximum compactness possible. Drastic increase in the number of electronic components per unit volume has led to ever-increasing amounts of heat fluxes. The electronics cooling (i.e. thermal management for microelectronic chips) focuses on various techniques for dissipating the heat generated in the microelectronics. The first generation of desktop computers used heat sinks. Nowadays, most of the desktop computers are using the cooling system that was first proposed for Pentium processors, which typically consists of a small aluminium heat sink with a fan (e.g. $60 \mathrm{~mm}$ in diameter) [1]. However, this cooling technique is not able to meet the requirements of the new microelectronic systems. In the next few years, the number of transistors per device is projected to reach $12 \times 10^{9}$ with a heat generation close to $200 \mathrm{~W}$. The current advanced technologies are able to transport heat fluxes in the order of $100 \mathrm{~W} / \mathrm{cm}^{2}$.

Accompanied by the rapid increase in the power of the computers, the size reduction, especially for notebook computers, is a limiting factor. For example, the Mac Book Pro, the latest generation of "Apple" notebooks, has a thickness of less than $2.5 \mathrm{~cm}$. Thus, the fast increase in the power and compactness of Central Processor Units (CPU), along with the decrease in space available for the cooling devices, stresses the need for a new generation of electronic cooling techniques.

\subsection{Enhanced heat transfer}

According to the second law of thermodynamics, the entire world is moving towards maximum entropy: "heat cannot of itself pass from a colder to a hotter body". By definition, "heat transfer is a basic science that deals with the rate of transfer of thermal energy" [2]. There are three basic mechanisms of heat transfer: conduction, convection, and radiation. Conduction is based on energy transfer between two adjacent particles of a substrate with different energy levels, whereas 
in convection, the heat transfers between a solid and an adjacent moving fluid. The mechanism of heat transfer through the emission of electromagnetic waves (or photons) from a matter is called radiation. Enhanced heat transfer in the industrial applications, such as electronics cooling, is often required. One of the most common methods of heat transfer enhancement is the use of enhanced surfaces, e.g. fins. Moreover, for a constant size and heat exchange rate, a lower temperature gradient shows a more efficient heat transfer. Enhanced heat transfer techniques can be classified as active and passive.

\subsubsection{Passive techniques}

Passive techniques do not require any external power and employ surface and fluid treatments to enhance heat transfer. Surface treatment techniques consist of surface coating or surface extension. Surface coating techniques use metallic or non-metallic coating. As an example of non-metallic coating, Teflon promotes dropwise condensation, while hydrophilic coatings promote the condensate drainage in evaporator. Fine-scale porous coatings enhance heat transfer by enhancing nucleate boiling [1]. Surface extension techniques use offset strip fins, segmented fins, integral strip-finned tubes; see Figure 1-1. These techniques decrease the thermal resistance by increasing the heat transfer coefficient $(h)$ or the surface area $(A)$. Fluid treatment techniques typically contain a number of geometrical arrangements to create a secondary flow. Some examples of fluid treatment techniques include the use of twisted-tape inserts, helical vane inserts and static mixers. Surface tension is typically employed to drive the working fluid in heat pipes. A wick structure in the heat pipe helps the capillary pressure to transport liquid films from the condenser to the evaporator. 


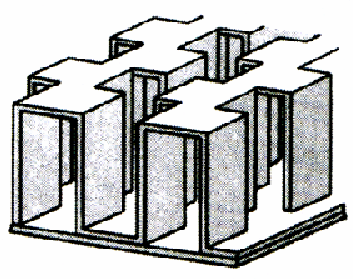

(a)

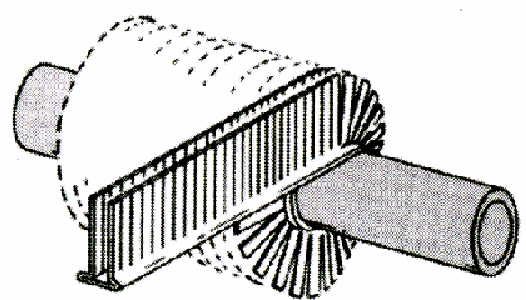

(b)

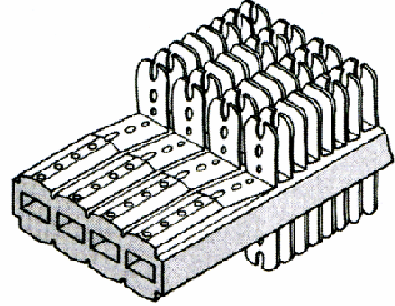

(c)

Figure 1-1: Enhanced surfaces: a) offset strip fins used in plate-fin heat exchangers, b) segmented fins for circular tubes, c) integral aluminium strip-finned tube [1].

\subsubsection{Active techniques}

Active techniques require external power, such as electronic or acoustic fields and vibration sources. In the electrostatic field technique, both direct current and alternative current can be applied to a dielectric fluid. That causes a better bulk mixing of the fluid in the vicinity of the heat transfer surface [1]. Vibration techniques are classified as surface vibration and fluid vibration techniques. Surface vibration impinges small droplets onto a heated surface to promote spray cooling. Both low and high frequencies are used in surface vibration, especially for single-phase heat transfer. However, fluid vibration is a more practical vibration enhancement, due to the mass of most heat exchangers. Surface vibration covers the frequency range from $1 \mathrm{~Hz}$ to ultrasound.

\subsection{Microfluidic chips}

Microfluidics, fluid mechanics at the micro scale, has received more attention in the past years due to ever-increasing applications. The adaptability of microfluidic devices has been a key factor in their wide range of applications. Thermal microfluidic chips employ the micro scale fluid flow for thermal applications. They have been considered for special applications such as electronics cooling and bio microelectromechanical systems. In microelectronics, thermal microfluidic chips provide attractive solutions for the thermal management in highly compacted integrated circuits. Increasing the compaction of electronic 
components requires tremendous amount of heat dissipation, i.e. in the order of $100 \mathrm{~W} / \mathrm{cm}^{2}$. Traditional techniques of heat removal, such as using cooling fans, cannot meet the thermal requirements of the new electronic chips. For cooling fans, further heat can be removed from a chip by increasing the fan's RPM. However, the frequencies of the noises generated by these fans pass the threshold of human hearing, and it disturbs the users.

In order to compare the heat transport capability of common electronic cooling techniques with those of thermal microfluidic chips, an "effective thermal conductivity" is defined as:

$$
k_{\text {eff }}=\frac{\left(Q_{T} / A\right)}{\left(T_{\max }-T_{\min }\right) / L_{e f f}}
$$

where $k_{\text {eff }}$ is the effective thermal conductivity, $Q_{T}$ is the total input heat, $A$ is the cross-sectional area, $L_{\text {eff }}$ is the characteristic length and $T_{\max }$ and $T_{\min }$ are the maximum and minimum temperatures, respectively [3]. A comparison between the effective thermal conductivity of common electronic cooling techniques and that of microfluidic chips is shown in Figure 1-2. In order to assist to a better comparison, thermal conductivities of some common materials are included in this figure.

According to Figure 1-2, microfluidic chips demonstrated very promising performances for heat removal applications. One very important benefit of using microfluidic chips for electronic cooling is the ability of manufacturing integrated microelectronic/microfluidic chips. Both active and passive techniques are used for different types of thermal microfluidic chips. Micro heat pipe, micro capillary pumped loop, micro loop heat pipe, micro gravitational heat pipe, and micro heat pips heat spreader are some examples of thermal microfluidic chips used in the various applications. 


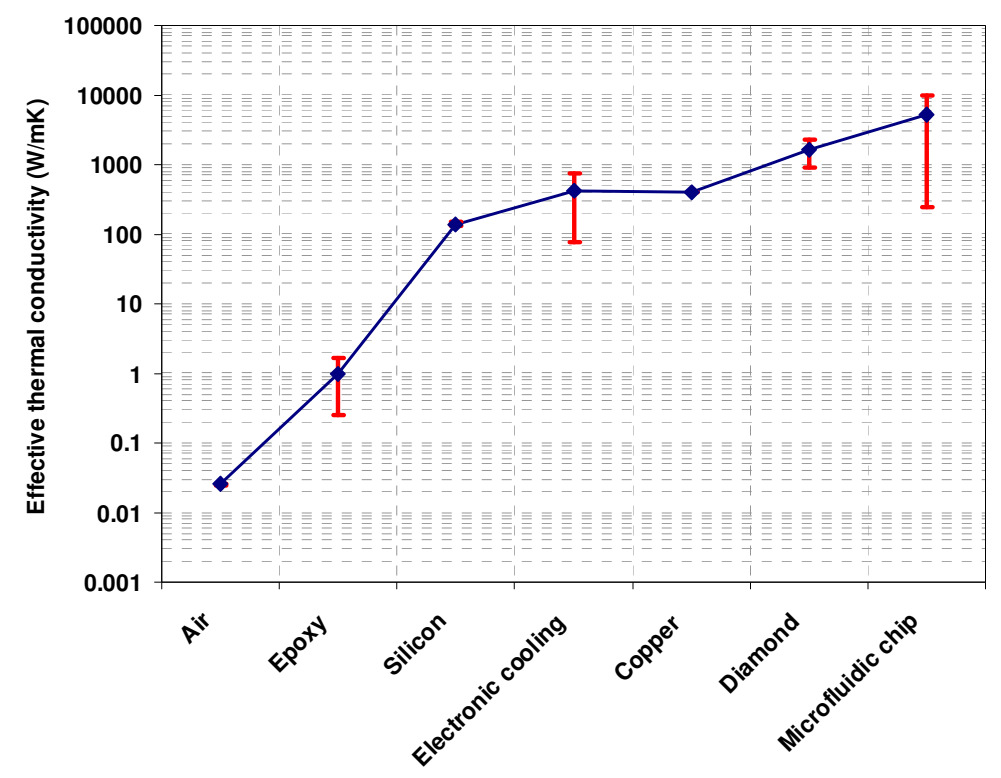

Figure 1-2: Effective thermal conductivity [2], [4]

\subsection{Project objectives}

The work presented here is part of a collaborative research project entitled "Design and assembly of 3-D micro heat pipe networks for heat dissipation in microelectronics". The objective of this project is the development and integrated corporations of three-dimensional micro heat pipes network to dissipate heat from electronic systems. The project is structured in three tasks:

1- Microfabrication and experimental testing

2- Thermal modelling

3- Integration and assembly for microelectronics

This thesis addresses the thermal modelling, where numerical and mathematical modelling is conducted for microfluidic chips. Thus, the objectives of this work are:

1- Numerical simulation of microfluidic chips using commercial finite element software for:

a. A single micro heat pipe 
b. Two-dimensional arrays of microchannels, i.e. planar microfluidic chip

c. Three-dimensional configurations of microchannels.

2- Optimization of the microfluidic chip geometric parameters

\subsection{Thesis organization}

The thesis is organized into five chapters. Chapter two focuses on a comprehensive literature review of the research carried out in the field of thermal microfluidic chips. It covers the analytical and experimental investigations as well as the manufacturing techniques. Chapter three discusses the micro modelling of a microchannel for single-phase and the two-phase flows. Chapter four provides a review of the macro modelling of two-dimensional and three-dimensional configurations of microchannels. It also includes the three-dimensional model of microfluidic chips under two-phase flow conditions. Chapter five is dedicated to the main conclusions along with a discussion on future research in this area. Finally, Appendix A briefly covers the derivation of the governing equations for coupled fluid flow/heat transfer phenomena in micro heat pipes. 


\section{Chapter 2 Literature review}

\subsection{Thermal microfluidic chips}

During the past two decades, the significant growth of microfluidic systems demonstrated promising capabilities for a wide range of applications. From drug delivery and biosensors in BioMEMS, to heat removal in microelectronic systems, microfluidic devices have proven their high efficiency and versatility. Microscale energy transport is an emerging science with a large number of potential applications [5]. More than 25 years ago, Tuckerman and Pease [4] presented the use of microchannels for electronics cooling. They investigated the thermal removal from planar integrated circuits, and used water as the working fluid in microchannels etched in a silicon substrate. The result was a heat transfer rate of $10^{5} \mathrm{~W} / \mathrm{m}^{2} \mathrm{~K}$ that was almost two orders of magnitude higher than state-ofthe-art commercial technologies for cooling Integrated Circuits [6]. Since then, experimental and theoretical investigations were conducted to address thermal behaviour of microfluidic chips. Research was carried out on single-phase flow microfluidic systems that are employing either gas or liquid [6], [7]. However, it was shown that the latent heat in a vaporization process can highly improve the efficiency of thermal microfluidic chips; hence, extensive research was carried out on two-phase flow thermal microfluidic devices [1], [6], [7], [8]. According to the literature, typical passive techniques were used in various microfluidic chips, such as micro heat pipes, micro heat spreaders, micro loop heat pipes, etc. Different investigations on fluid flow and heat transfer in microfluidic devices were compared analytically [9].

\subsubsection{Micro heat pipe}

Three years after employing microchannels for electronics cooling by Tuckerman and Pease [4], Cotter [10] introduced micro heat pipes as "one so small that the 
mean curvature of the vapour-liquid interface is necessarily comparable in magnitude to the reciprocal of the hydraulic radius of the total flow channel".

According to Figure 2-1, the operating principles of micro heat pipes are identical to their larger counterparts, heat pipes [11], [12]. The vaporization of the working fluid in the evaporator section absorbs heat from the eat source. The vapour moves towards the condenser, where it condenses and releases its latent heat to the heat sink. Unlike the conventional heat pipes, micro heat pipes use sharp edges rather than wick structures. These sharp edges function as arteries to bring back the liquid from the condenser to the evaporator, as a result of capillary pressure difference. That causes continuous liquid and vapour flows in opposite directions, i.e. the liquid flows from the condenser to the evaporator in the sharp corners, while the vapour moves from the evaporator to the condenser in the central parts of micro heat pipe.

Cotter [10] emphasized that the maximum value of the mean radius of curvature can be used to characterize the capillary effects. Babin et al. [13] presented a mathematical expression for Cotter's definition of micro heat pipes, as:

$$
K \propto \frac{1}{r_{h}}
$$

where $K$ is the mean curvature of the liquid-vapour interface, and $r_{h}$ is the hydraulic radius of the flow channel. Peterson [11] proposed unity as the constant of proportionality and multiplied both sides by the capillary radius, $r_{c}$, to obtain:

$$
\frac{r_{c}}{r_{h}} \geq 1
$$

This definition helps to distinguish the "micro heat pipes" from the small version of conventional heat pipes. However, micro heat pipes and the conventional heat pipes are identical in a number of aspects. Significant investigations in the field of micro heat pipes [14], [15], along with the well established knowledge of 
conventional heat pipes [11], [12] has brought a solid foundation to this field of science.

The operation and performance of micro heat pipes depend on several characteristic parameters, including the channels geometry and the working fluid thermal properties. Similar to conventional heat pipes, micro heat pipes have borders that define their operation. Some of these limitations include capillary, viscous, sonic, entrainment, and boiling limitations.

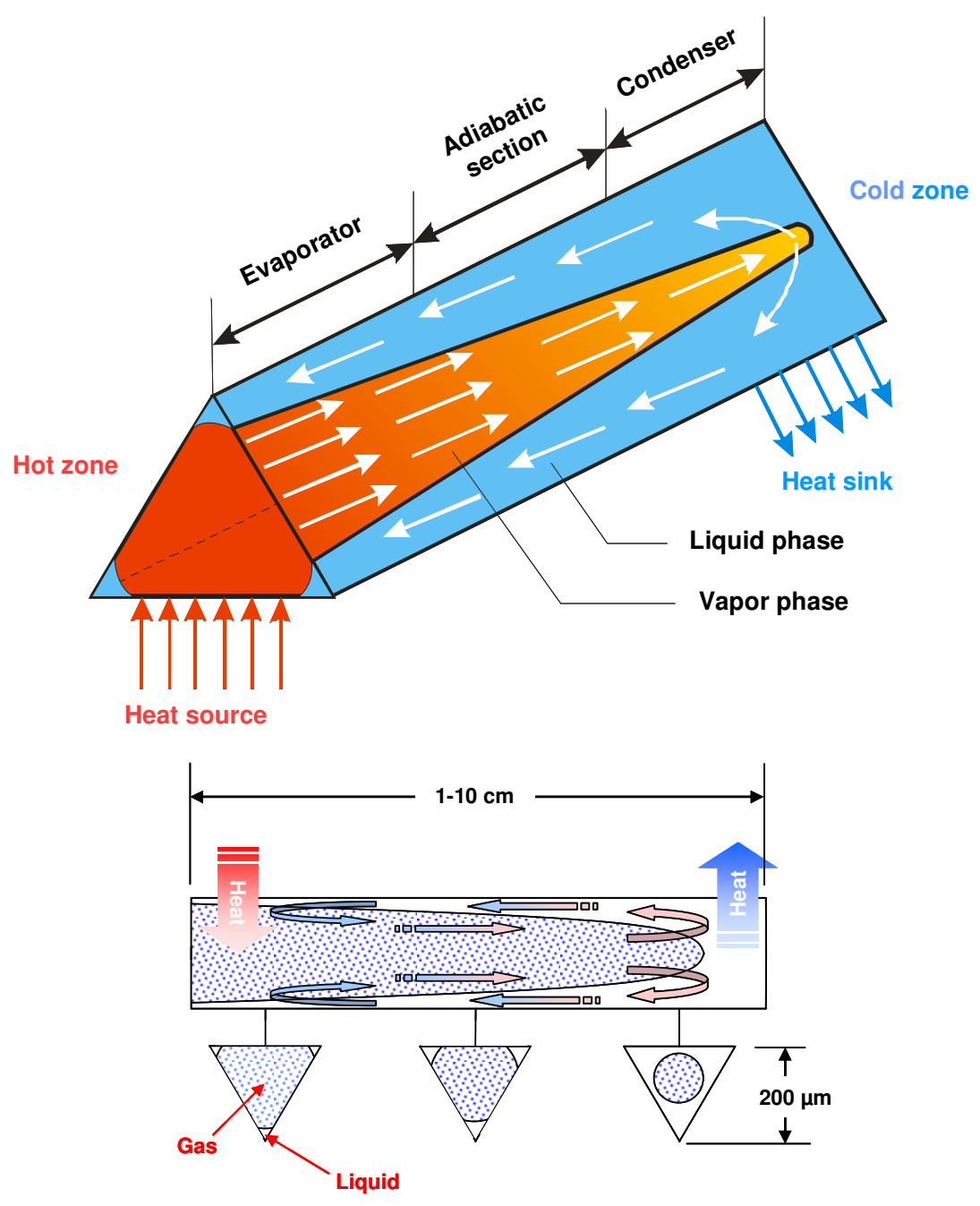

Figure 2-1: Micro heat pipe operation 
In order for a micro heat pipe to work properly, the capillary pressure difference at the liquid-vapour interface in the evaporator and condenser must be greater than the sum of all pressure losses along the liquid and vapour flows [12]. The hydraulic pressure drop and the viscous pressure drop are the two major sources of pressure loss in micro heat pipes.

Viscous limitation occurs at very low operating temperatures, where the vapour pressure difference between the evaporator (i.e. the high pressure region) and the condenser (i.e. the low pressure region) is small. In these situations, the vapour pressure gradient within the microchannel is not sufficient to overcome the viscous forces in the vapour region, and the vapour phase cannot flow in the micro heat pipe to complete the circulation of the phases. The thermophysical characteristics (e.g. temperature and pressure difference) that define the viscous limitation depend on the working fluid.

According to sonic limitation, the axial velocity of vapour flow within a micro heat pipe cannot exceed the speed of sound, which causes the flow to be chocked.

In case of very high heat fluxes the micro heat pipe may not operate properly, due to entrainment limitation. In this situation, there will be a high shear stress between vapour and liquid flows, which are in opposite directions. That may cause the liquid droplets to be picked up or entrained in the vapour flow. Consequently a dry-out of the evaporator happens due to the excess accumulation of liquid in the condenser.

Boiling limitation may also happen in case of high heat fluxes in the evaporator. The nucleate boiling in the evaporator section causes the bubbles to block the return of liquid, and decrease the thermal performance of the micro heat pipe. 


\subsubsection{Micro heat spreader}

Micro heat spreaders, or micro heat pipe heat spreaders [16], are passive thermal microfluidic chips that work with the same principle as micro heat pipes. Micro heat spreaders are typically planar circular structures with layers of radial channels [17]. Schematic configuration and operation of a typical micro heat spreader are shown in Figure 2-2. Heat is applied to one side of the plane in the central part. Vaporization takes place and causes the vapour to flow to the other layer in the central part, and then through radial channels towards the circumference of the circular structure. In the circumferential area, the vapour is condensed and flows back to the bottom layer. The liquid, then, flows toward the central part as a result of capillary pressure [16], by means of a wick structure [18].

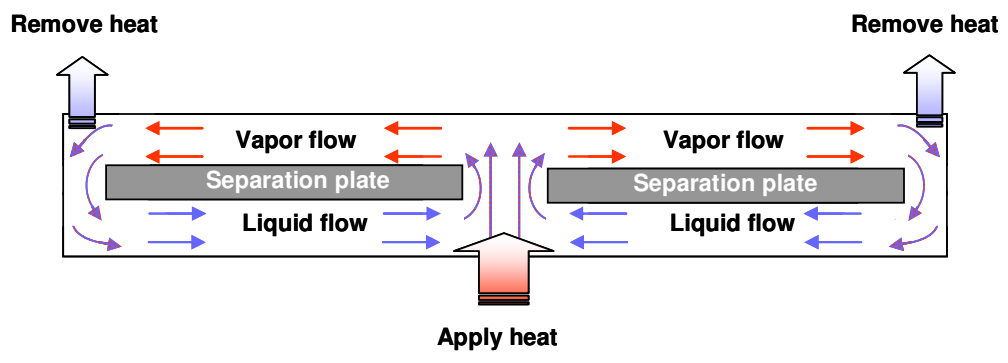

(a)

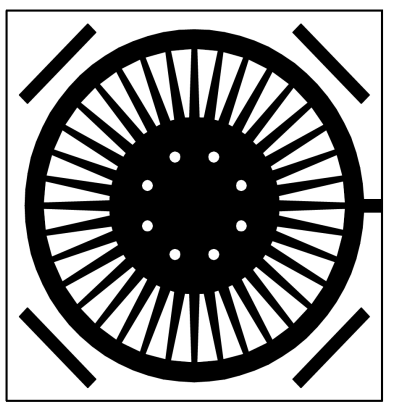

(b)

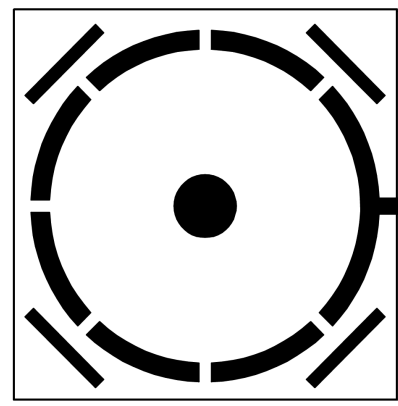

(c)

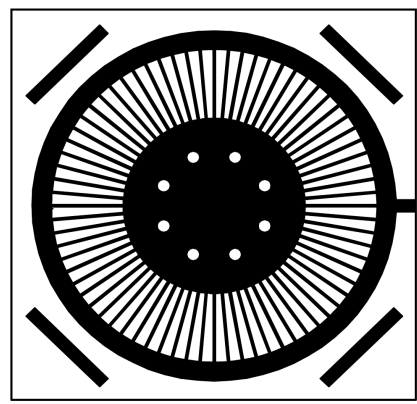

(d)

Figure 2-2: Micro heat spreader, a) operation, b) vapour flow layer, c) mid-layer, d) liquid flow layer [16].

The operating limitations of micro heat spreaders depend on the design and characteristic parameters of the system. For instance in some designs [16], a 
separation plate is placed between the top and bottom layers of micro heat spreader, which removes all concerns about the entrainment limitation.

\subsubsection{Micro gravitational heat pipe}

The term "gravitational heat pipe" is applied to heat pipes that operate at an inclination angle with respect to a horizontal plate; thus, gravity affects their operation and performance [19]. For small gravitational heat pipes, the term of "mini" or "micro" may be used depending on the channel cross-section geometry. A mini gravitational heat pipe has a circular cross-section and therefore the operation of the heat pipe is primarily based on gravitational forces. On the other hand, the channel sharp edges, in a micro gravitational heat pipe typically cause the working fluid to flow both from gravitational and capillary forces. Thus, the primary difference between mini and micro gravitational heat pipes is the nonnegligible effect of capillary and viscous forces in micro gravitational heat pipes. Micro and mini gravitational heat pipes have been investigated mostly because of their application in solar collectors [19].

\subsubsection{Micro loop heat pipe}

Conventional heat pipes have demonstrated their high performance for different applications. However, a serious reduction in the thermal efficiency of conventional heat pipes rises when the condenser is located bellow the evaporator. Loop heat pipes and capillary pumped loops are the solution for this problem [12]. Analogous to conventional loop heat pipes, a micro loop heat pipe consists of an evaporator, a condenser, a compensation chamber and fluid lines. Figure 2-3 shows a typical micro loop heat pipe. The working fluid absorbs heat in the

evaporator and vaporizes. The vapour flows to the condenser through a vapour line. The absorbed heat is then released during a condensation process. Afterwards, the liquid moves towards the compensation chamber through a liquid line. The compensation chamber is attached to the evaporator by means of capillary microchannels to play two important roles; firstly, the compensation 
chamber adjusts the amount of liquid and prevents the liquid to accumulate in the condenser. Secondly, it insures a continuous flow of liquid in the evaporator and prevents dry-out in case of sudden increase of the heat flux in the evaporator [20].
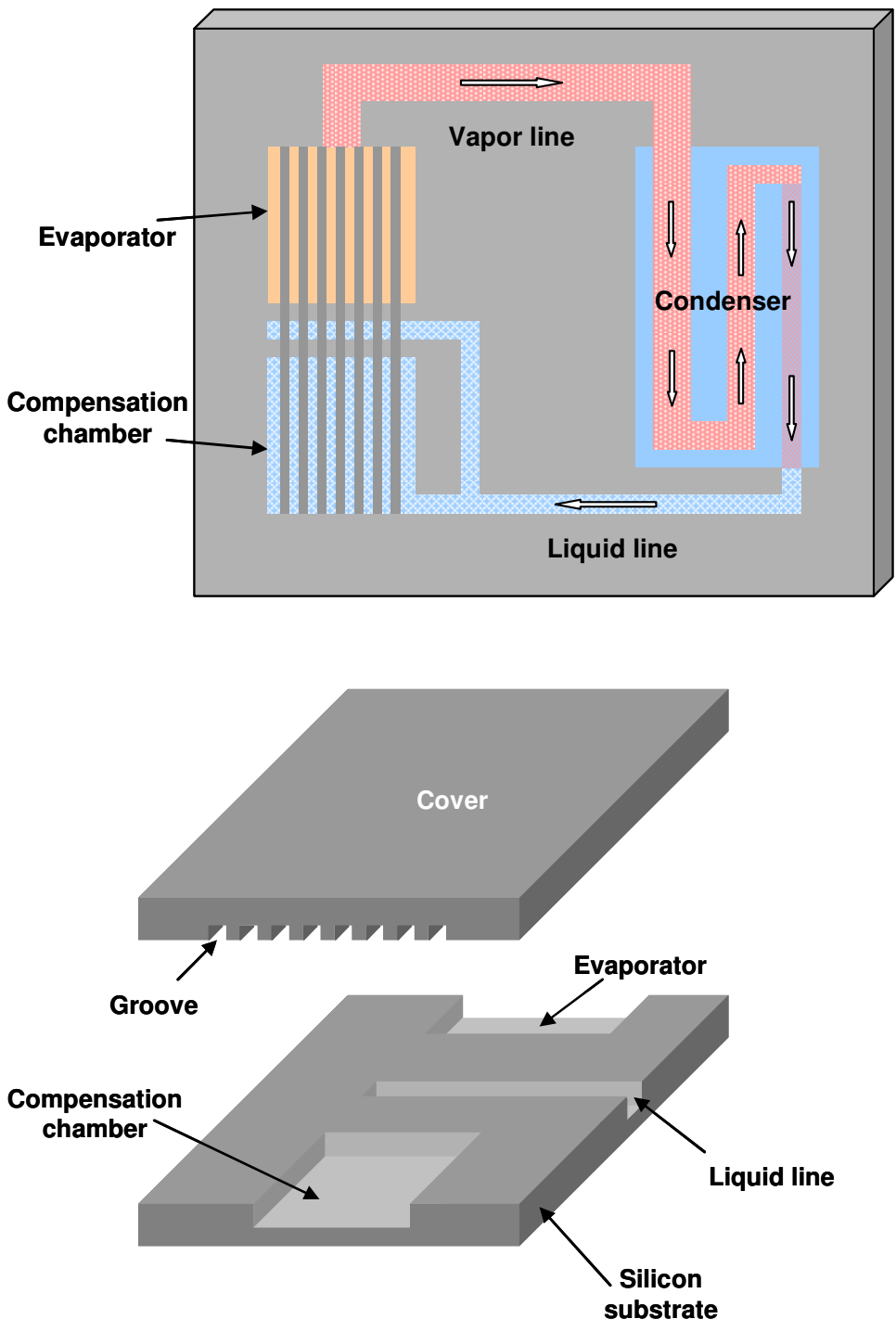

Figure 2-3: Micro loop heat pipe [20]

Micro loop heat pipes have been investigated for electronics cooling application, and demonstrated promising performances [21], [22]. The main advantage of these devices is the ability to withstand the unexpected changes in the evaporator heat fluxes. Furthermore, micro loop heat pipes are passive devices and there is 
no need for external power to drive the cooling system. Given that the fundamental operating principles of micro heat pipes and micro loop heat pipes are identical, the operational limits of these two devices are similar. However, in micro loop heat pipes, liquid and vapour transport lines are separated, and therefore there is no entrainment limitation.

\subsection{Microfluidic modelling}

Attempts to model different types of microfluidic devices were initiated since the first introduction of thermal microfluidic chips, more than 25 years ago [4]. During the past years, early one-dimensional models were replaced by two or three-dimensional analyses. Single-phase studies were improved to predict the phase change and two-phase models were proposed. Both steady state and transient models were improved to predict the thermal performance of microfluidic chips.

\subsubsection{Steady state}

The first steady state model of micro heat pipes was developed by Cotter in 1984 [10]. A definition for micro heat pipes was also proposed and restricted the configuration of interest to channels of a few centimetres long with convex yet cusped cross-sections and a diameter in the range of about 10 to $500 \mu \mathrm{m}$. In 1997, $\mathrm{Ma}$ and Peterson [23] presented a semi-empirical correlation between the experimental and predicted results, and reported the input power as a function of the operating temperature. They re-evaluated the original analytical model presented by Cotter [10] and demonstrated that it significantly over-predicted the maximum heat transport capacity. 


\subsubsection{Evolution of models}

Following Cotter [10], Longtin et al. [24] carried out a one-dimensional analysis of a micro heat pipe operating under steady state conditions. They used the governing equations of Laplace-Young, conservation of mass, momentum and energy; and obtained a set of five first-order, nonlinear, coupled ordinary differential equations. The equations were solved numerically and the results showed that the maximum heat transport capability of a micro heat pipe was a function of the inverse of its length and the cube of its hydraulic diameter. Longtin et al. reported pressure and velocity for both liquid and vapour, as well as the liquid/vapour interfacial characteristics as functions of the axial distance along the device; see Figure 2-4.

Different research groups focused on two-dimensional models for micro heat pipes. Stephan and Busse [25] proposed a radial heat transfer model for grooved heat pipe evaporators and calculated the shape and the temperature of the liquid/vapour interface. Ma and Peterson [26] reduced the governing equations to a third-order ordinary differential equation and solved it using a forth-order Runge-Kutta method. It was found that for a constant heat flux boundary condition, the heat transfer coefficient decreased and the wall temperature increased in the channel direction. Moreover, the authors demonstrated that the apparent contact angle increases with increasing superheat and therefore the heat transfer rate through the micro region increased along the axial direction. The model, also, demonstrated that the effect of the surface tension variation caused by temperature increase in the micro region could be neglected.

Sartre et al. [27] carried out a three-dimensional steady state investigation on micro heat pipes. Three coupled models were required in order to obtain the main characteristics (e.g. temperature) of the field: the two-dimensional wall heat conduction, and the longitudinal capillary two-phase flow. The results showed that both the contact angle and the heat transfer rate increased with an increasing 
wall superheat. Additionally, the authors reported the contact angle as a function of the temperature difference between the wall and the interfacial vapour.

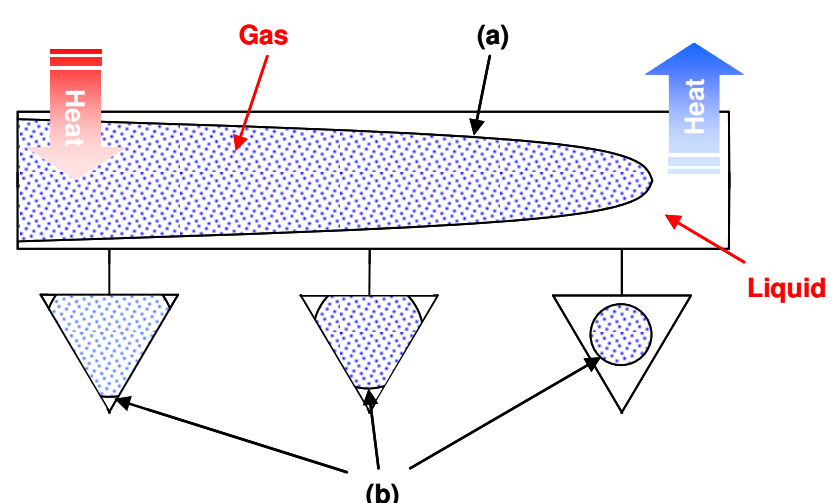

(b)

Figure 2-4: a) The one-dimensional interface addressed by Longtin et al. [24]. b) The twodimensional interfaces between liquid and vapour addressed by Stephan and Busse [25].

Recently, research has been carried out to model microfluidic chips, using finite element software. In 2004, Tiselj et al. [28] focused on numerical and experimental analysis to explore the effect of axial conduction on the heat transfer in microchannels. They developed a single-phase flow in a three-dimensional numerical model including equilateral triangular microchannels with a side of 310 $\mu \mathrm{m}$ and a channel length of $10 \mathrm{~mm}$ using the computer code CFX-5. The temperature contours in the microchannels, surrounding silicon and the walls were reported, and the values of heat transfer were determined based on the mass flow rate in the microchannels.

\subsubsection{Thermal capacity}

One of the primary characteristics of thermal microfluidic chips is their thermal capacity. Different research groups investigated the effect of various parameters on the thermal capacity of microfluidic chips. In 1994, Khrustalev and Faghri [29] demonstrated the importance of the liquid filling ratio, minimum wetting contact angle, and the shear stress at the liquid/vapour interface on the maximum heat transfer capacity of micro heat pipes. Furthermore, Peterson and Ma [30] 
investigated the effect of minimum meniscus radius on the maximum heat transport capacity in triangular grooves. The same authors considered the temperature distribution along the axial direction of the heat pipe and evaluated its effect on the heat transfer. They reported the meniscus radius and heat transfer as a function of the hydraulic diameter. The results indicated that the heat transport capacity of micro heat pipes was strongly affected by the apex angle of the liquid arteries, the contact angle of the liquid flow, the length of the heat pipe, the vapour flow velocity and the inclination angle.

$\mathrm{Wu}$ and Peterson [31] experimentally validated the model proposed by Khrustalev and Faghri [29]. They demonstrated that for nearly maximum thermal loads, a very high fraction of the liquid pressure drop occurred in the evaporator and the beginning of the adiabatic section. It was also shown that the dominant thermal resistances within the micro heat pipe were those of the vapour flow and liquid film in the evaporator and condenser. Peterson and Ma [32] showed that the temperature drop occurring in the evaporator was considerably larger than in the condenser.

\subsubsection{Dry-out phenomenon}

Babin et al. [13] performed steady state modelling and experiments on micro heat pipes and demonstrated that the model was strongly dependent on the way dry-out was defined. Ha and Peterson [33] performed an analytical investigation on the vaporization in triangular grooves to predict the axial dry-out point. They predicted the axial dry-out location as a function of the input heat flux, thermophysical properties of the system, and geometric parameters of the grooves.

Further investigations were carried out to determine the dry-out phenomenon for different geometries. Peterson et al. [34] considered the rewetting characteristics of capillary induced liquid flow in circular channels with small circumferential 
grooves machined on the inner surface. Schneider et al. [35] studied interconnected mini heat pipe arrays for micro electronic cooling. They showed that when the heat input power was increased past the dry-out limit, the evaporator temperature increased rapidly.

The effects of dry-out on the capillary limit and the maximum capacity for different geometries of micro heat pipes were demonstrated before. Therefore, Suman et al. [36] proposed a model to address the capillary limit of a micro heat pipe and predicted the dry-out length for any polygonal shape. They solved the governing equations for fluid flow and heat transfer and discussed the effect of inclination and number of sides of a polygon on the critical heat input. Based on the model, the dry-out length versus the input power was also evaluated. Suman et al. concluded that the effect of body forces was small, and an increase in the apex angle reduced the capillary pressure. Suman and Kumar [37] also developed an analytical model and solved the governing equations analytically. They compared the results with those of the previous research [36] and demonstrated an error of less than $3 \%$.

Recently, Suman and Hoda [38] investigated an array of ten heat pipes with 100 $\mu \mathrm{m}$ widths and $100 \mu \mathrm{m}$ gaps between the channels. They proposed a new method to calculate the dry-out length. Due to some difficulties in the fabrication of very sharp apex angles, the authors addressed the effect of roundness in the corners of the microchannels. Based on the modelling results, the effect of the substrate temperature on the thermal performance of micro grooved heat pipe was investigated.

\subsubsection{Geometry}

There are various investigations in the literature that considered a given geometry and studied the thermal characteristics of that given configuration of microfluidic 
chip [36], [34]. However, few researchers addressed the effect of geometric parameters on the different characteristics of microfluidic chips.

Dickey and Lam [39] investigated the impact of channel geometry on the heat transfer in microchannels for high density electronics cooling. They only considered equilateral triangular grooves in silicon substrate and carried out numerical simulations to evaluate the effects of geometric parameters on the heat transfer. They considered water as working fluid in a single-phase pumped loop, and reported the average surface temperature as a function of gap distance (from near zero to $60 \mu \mathrm{m}$ ) between channels. In addition, temperature contours for gap distances of $1,10,20$, and $40 \mu \mathrm{m}$ were plotted. Based on the numerical models, a minimum average surface temperature was reached for a gap distance of $6 \mu \mathrm{m}$ and an equilateral triangular cross-section with sides of $200 \mu \mathrm{m}$. Dickey and Lam indicated that the variation of average surface temperature between $200 \mu \mathrm{m}$ and $300 \mu \mathrm{m}$ sided triangles was within $5 \%$, provided the gap distance was between 5 and $15 \mu \mathrm{m}$.

$\mathrm{Li}$ and Peterson [40] proposed a detailed numerical simulation in order to optimize the geometric structure of silicon-based microchannel heat sinks. They used a three-dimensional conjugate heat transfer, i.e. two-dimensional fluid flow and three-dimensional heat transfer. They considered arrays with 40 to 140 rectangular microchannels with widths between 20 and $220 \mu \mathrm{m}$ and depths between 100 to $400 \mu \mathrm{m}$. They presented their results in the form of thermal resistance and addressed the effect of microchannel geometry on the temperature distribution in the microchannel heat sink. The optimum thermal performance was obtained for 120 channels per centimetre with an aspect ratio as large as possible (i.e. very deep grooves). 


\subsubsection{Transient}

Due to the unstable nature of the two-phase flow in micro heat pipes, it is complicated to consider all intricate phenomena in a steady state model. It will certainly add more difficulties to the model, if the characteristic parameters of a coupled fluid flow/heat transfer process vary with time, i.e. for transient conditions. In order to capture the transient behaviour of micro heat pipes, researchers have used simplifying assumptions.

As early as 1991, Wu and Peterson [31] proposed a transient numerical model to predict the thermal behaviour of the micro heat pipes during start-up or variation in the evaporator thermal load. The authors described mass flow rate, pressure, and liquid cross-section as functions of axial position and time, and concluded that unlike traditional heat pipes, reverse liquid flow occurred in the liquid channels during start-up. In addition, they demonstrated that the wetting angle was one of the most important factors affecting the transport capacity and behaviour of micro heat pipes.

Suman et al. [41] developed a one-dimensional transient model to address fluid flow and heat transfer for any channel with polygonal cross-section. The model consisted of ten micro grooves of width and spacing distance of $200 \mu \mathrm{m}$. The authors assumed that the shear stress at the liquid-vapour interface was negligible. Thus, they could study the effect of the groove dimensions and heat input on the characteristic parameters (e.g. pressure and temperature) of micro grooved heat pipes. The variation of dimensionless interface radius of curvature with the time, and the magnitude of the liquid velocity as a function of time and position were predicted. It was found that the steady state temperature was reached in less than $20 \mathrm{~s}$. However, the transient profiles for the liquid velocity and the liquid pressure were increasing with the time and reached steady state in $10 \mathrm{~s}$. 


\subsection{Microfluidic testing}

Beside the modelling research, experimental investigations have been carried out to validate the models and provide an understanding of the complicated phenomena inside microfluidic chips. The challenges of the experiments arise from small size of the specimens, the precision of the measurements and visualization of the phenomena. Early experiments were focused on the demonstration of the viability of thermal microfluidic chips, whereas recent investigations aimed at the optimization of these devices.

\subsubsection{Steady state}

The first experiments were performed to evaluate the early proposed models. Once Cotter [10] proposed his model, Babin et al. [13] built an experimental setup to validate the results of the steady state modelling. They studied two micro heat pipes made up of copper and silver with $1 \mathrm{~mm}^{2}$ in cross-sectional area and $57 \mathrm{~mm}$ in length. They found that Cotter [10] can predict the trends correctly, with the accurate estimation of the integrated fraction of axial heat transfer.

\subsubsection{Array of microchannels}

Most of the experimental investigations considered array of microchannels, rather than single microchannels, to study the thermal behaviour of microfluidic chips. In 1993, Peterson et al. [42] carried out an experimental investigation on arrays of micro heat pipes fabricated in silicon wafers. They selected methanol as working fluid and manufactured two types of micro heat pipe arrays, rectangular and triangular channels. In order to consider the efficiency of micro heat pipes, the authors compared the experimental results with those obtained from a plain silicon wafer without micro heat pipes. They concluded that micro heat pipes significantly increased the effective thermal conductivity, and decreased both the maximum temperature and temperature gradient within the wafer. 
Launay et al. [3] experimentally studied silicon micro heat pipe arrays for electronic cooling purposes. Two arrays of 55 and 25 triangular microchannels were filled with ethanol and methanol, respectively. They increased the effective thermal conductivity of the system from 140 (thermal conductivity of silicon) to $300 \mathrm{~W} / \mathrm{mK}$.

\subsubsection{Flow observation}

Research has been conducted to investigate the phase change phenomenon and visualize the two-phase flows inside the microchannels. The predicted profile for the liquid/vapour interface was evaluated by experimental studies. Chung et al. [43] considered the effect of channel geometry on the two-phase flow through square and circular microchannels. In an adiabatic situation, they pumped a waternitrogen gas mixture through a $96 \mu \mathrm{m}$ square microchannel to consider the flow pattern, void fraction and frictional pressure drop. Images of the flow patterns were captured during the experiment. Chung et al. only observed slug flow and did not detect any bubbly, churn, or annular flow in the tested microchannels. They concluded that the absence of bubbly and churn flow patterns could be attributed to the laminar nature of liquid flow in the microchannels.

In 2003, Lee et al. [44] fabricated and characterized a micro heat pipe system with triangular cross-section. They used silicon-to-glass bonding technique to fabricate the system. The two-phase flow pattern was observed during operation, while the evaporator, adiabatic section and condenser were clearly distinguishable, see Figure 2-5.

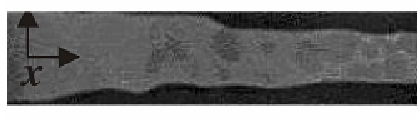

(a)

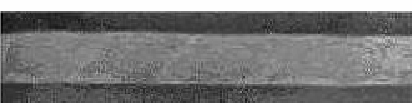

(b)

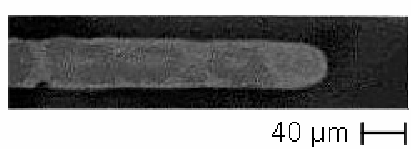

(c)

Figure 2-5: The flow pattern at a) evaporator, b) adiabatic section, and c) condenser 
Experimental analysis on micromachined silicon channels with hydraulic diameters in the range of $27-171 \mu \mathrm{m}$ was performed by Zhang et al. [45]. They manufactured microchannels with varying surface roughness in order to explore phase change phenomena. Bubble nucleation, flow patterns, and wall temperature, along with transient pressure fluctuations were recorded and analyzed. Zhang et al. could not observe bubbly and slug/plug flow regimes in their experiments. They concluded that a single bubble could quickly grow to the size of the channel diameter and suppress the two regimes.

Hetsroni et al. [46] performed flow visualization by means of a high-speed video recording system. The results showed that for cooling by Vertrel XF, the maximum temperature difference on the surface did not exceed 4-5 K, while the water cooling system had a maximum temperature difference of $20 \mathrm{~K}$, at a comparable flow rate.

\subsubsection{Thermal capacity}

One of the main objectives in the field of thermal microfluidic chips is increasing the thermal capacity of these devices. Significant research has been carried out to investigate various parameters that affect the thermal capacity. Ma and Peterson [47] experimentally investigated the capillary heat transport limit at steady state. They selected methanol as working fluid for an array of ten grooves and reported the thermal capacity as a function of the hydraulic diameter. A good agreement was found with the models [30] developed previously.

Another experimental study on thermal capacity of micro heat pipes with polygonal cross-section was accomplished by Moon et al. [48]. The researchers studied triangular and rectangular cross-sections, and selected $20 \%$ filling ratio of water, as working fluid. The experiments were performed in a vacuum chamber in order to minimize the heat losses through convection. Although the authors did 
not observe any significant effect of inclination angle on the heat transfer, they suggested having evaporator in a lower height compared to the condenser.

Moon et al. [49] used miniature pipes with $2 \mathrm{~mm}$ diameter, made up of copper, in order to improve thermal performance of miniature heat pipe for notebook PC applications. The authors demonstrated the importance of the liquid filling ratio on the thermal performance of the miniature pipes. The authors also concluded that the heat transfer limit was dependent on the inclination angle, indicating that the cooling performance of the heat pipe was influenced by gravity.

\subsubsection{Liquid filling ratio}

The importance effect of liquid filling ratio on the thermal performance and maximum capacity of microfluidic chips was demonstrated [49]. Hence, the optimization of liquid filling ratio for a triangular micro heat pipe was performed by Duncan and Peterson [50]. They used methanol as working fluid and employed an anisotropic etching process to produce a series of 59 parallel triangular channels. Four different liquid filling ratios were examined: 10, 20, 30, and 50\%. The results demonstrated that the effective thermal conductivity continued to increase with increasing liquid charge. However, Launay et al. [3] examined the influence of liquid filling ratio on the effective thermal conductivity, and suggested the fluid charge of $24 \%$ to reach the optimum condition.

In 1999, Schneider et al. [35] manufactured 20 interconnected triangular grooves into an aluminium-metal-ceramic base plate and used water to fill the channels. Three different liquid filling ratios were tried: $67 \%, 50 \%$ and $10 \%$. The results implied the interaction processes between the grooves. It was fount that the used wick structure could strongly affect the liquid distribution within the mini heat pipe arrays. 
Kang et al. [51] proposed a three-layer radial structure of grooved micro heat pipes in order to separate the liquid and vapour flows and reduce the viscous shear force. Radial grooved micro heat pipes that were filled with $70 \%$ fill rate showed the best performance. The performance was determined by the heat flux or minimum temperature difference at which a certain amount of heat can be transferred.

\subsubsection{Transient}

Successful transient experiment requires the monitoring of characteristic parameters during the transient time. Transient times of various phenomena in microfluidic devices are typically short (i.e. less than a minute), and a change in boundary conditions rapidly leads to a new steady state situation, where the parameters are independent of time. Exploring time dependent parameters during an experiment adds new challenges to the test.

$\mathrm{Wu}$ et al. [52] performed a transient experimental investigation in tapered micro heat pipes. They verified the operation, measured the performance limits, and determined the accuracy of a previously developed numerical model [31]. The test results demonstrated that the previous model was capable of predicting the maximum heat transport capacity prior to the onset of dry-out. The predicted values for temperature distribution throughout the longitudinal position, and the temperature difference between axial locations on the heat pipe were accurate within a $0.3^{\circ} \mathrm{C}$ difference. The authors concluded that although the developed numerical model accurately predicted the steady state behaviour, it substantially underestimated the transient response.

\subsection{Manufacturing techniques}

The first microfabrication techniques were developed to meet the requirements of microelectronics. Later on, the developments in fabrication of integrated circuits 
(IC) extended to be employed in fabrication of microelectromechanical systems (MEMS) [6]. Currently, thermal microfluidic chips are typically manufactured with the fashionable MEMS manufacturing techniques, such as photolithography. Wet and dry etching, sputtering, and chemical vapour deposition [53] can be mentioned as examples of MEMS manufacturing techniques. Experimental results [42] have demonstrated that integration of microfluidic chips with semiconductor devices has several advantages, including the increase in thermal conductivity and decrease in temperature gradient across the device. Thus, research has been performed on various integration techniques, such as bonding [16].

As substrate material for most of microfluidics manufacturing techniques, silicon has a number of promising characteristics, including relatively high thermal conductivity, $140 \mathrm{~W} / \mathrm{mK}$. Thus, significant research has been carried out on different manufacturing aspects of silicon microfluidic chips [51]. However, for some applications in which the heat loss is critical, e.g. sensors, high conductivity of silicon is a disadvantage. Therefore, low conductive materials, like epoxy resins) have also been used for microfluidic chips manufacturing [54].

A majority of investigations of microfluidic chips had been restricted to planar configurations. However, Therriault et al. [55] proposed a three-dimensional network of microchannels manufactured with the new technique of direct-write assembly. They proved the capability of the new method to manufacture smooth cylindrical channels in the range of 10 to $300 \mu \mathrm{m}$. The manufacturing technique consisted of four steps, Figure 2-6. The first step included two or threedimensional deposition of a fugitive ink through a nozzle on a substrate, by means of a robotic system. In the second step, the entire scaffold was infiltrated by an epoxy resin. After the solidification of the resin in the third step, in the fourth step the fugitive ink was removed to create an interconnected network of microchannels inside the epoxy block [56]. The three-dimensional network of microchannel was able to demonstrate promising characteristics for thermal microfluidic devices [57], [58]. The high effective conductivity of the 
microchannels along with the ability of manufacturing three-dimensional network removed the drawback of low thermal conductivity in the surrounding epoxy. Thus, a geometric optimization of the microchannels was conducted by Rahmat and Hubert [59]. However, if a higher conductivity in the surrounding epoxy is required, different techniques of increasing thermal conductivity of the epoxy [60], [61], [62] can be employed. Kimura et al. [61] proposed silver particles in the range of 1-10 $\mu \mathrm{m}$ to fill the epoxy resin. They achieved the maximum thermal conductivity of $65.8 \mathrm{~W} / \mathrm{mK}$ for a volume fraction of $60.5 \%$. However, high volume fraction of silver particles in the epoxy may increase the viscosity of the mixture and cause some difficulties in the direct-write method [55].
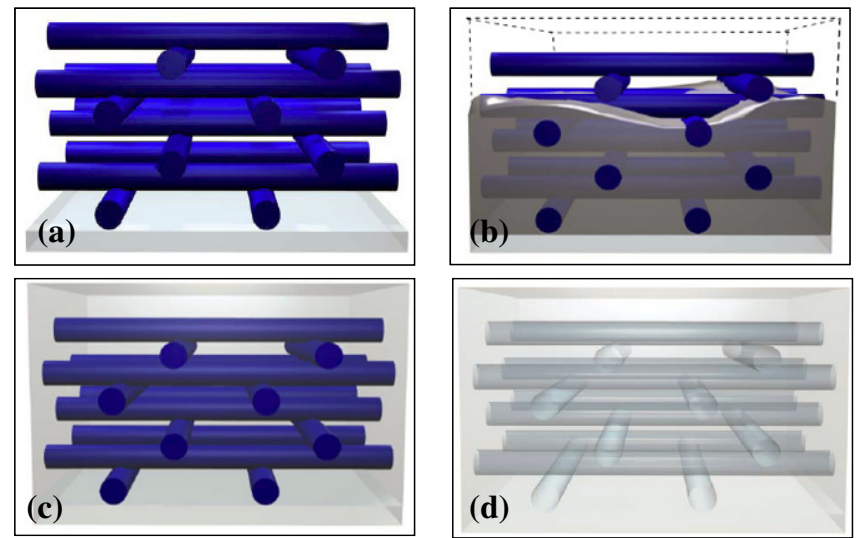

Figure 2-6: Direct-write technique, a) ink deposition, b) resin infiltration, c) resin solidification,

d) ink removal [56]

\subsection{Summary and thesis approach}

As presented in the previous sections, significant research has been performed on different aspects of microfluidic systems. A brief summary of various investigations is shown in Figure 2-7. According to Figure 2-7-a, almost the same amount of research has been dedicated to experimental investigations and modelling approaches. Experiments assist to validate the modelling predictions and validated models can be used to limit costly experiments. Figure 2-7-b presents the working fluids that are used in the various studies. Water have been the most popular working fluid in the filed of thermal microfluidics due to its 
promising thermophysical properties. High amounts of latent heat of vaporization (for two-phase flows) and specific heat (in case of single-phase flows) have made water an excellent option in thermal applications. On the other hand, silicon has been quite attractive as a substrate for microfluidic chips, Figure 2-7-c. The reasons for this fact lie in its high thermal conductivity and adoptability to be integrated with the microelectronics chips. Finally, Figure 2-7-d compares different cross-sections that are mostly used for microchannels in thermal microfluidic devices. The need for sharp edges, especially for capillary driven microfluidic devices, causes the triangle to be the most popular cross-section for microchannels.
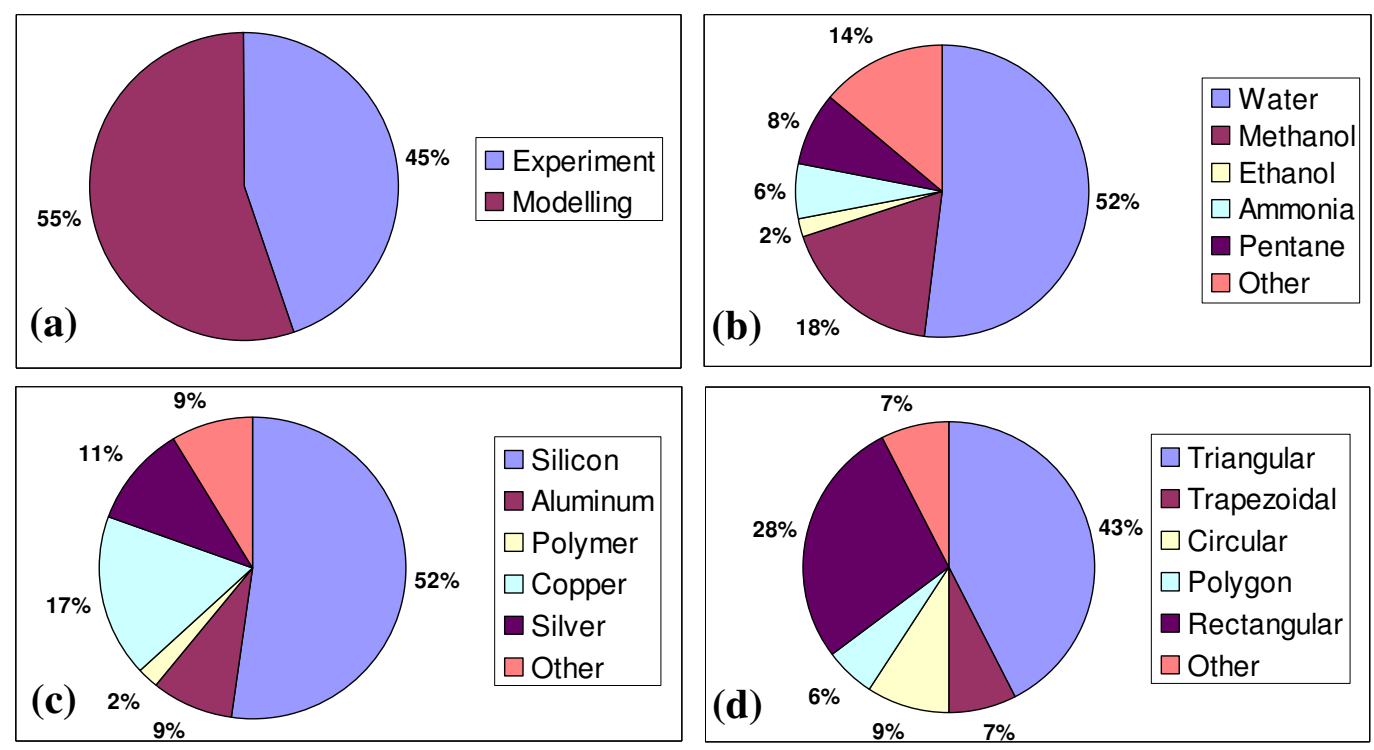

Figure 2-7: a) Investigation method, b) Working fluid, c) substrate, d) channel cross-section

A number of investigations considered the effect of geometry on the thermal characteristics of microfluidic devices. However, there is no general optimization for the geometric parameters of thermal microfluidic chips presented in the literature. Previously performed optimizations [39], [40] only dealt with one specific channel cross-section, and tried to obtain the best thermal performance by changing the geometric parameters. There is no general comparison between different channel cross-sections to present the optimum cross-section for different 
applications. Moreover, since the idea of manufacturing three-dimensional configuration of microchannels has been proposed recently, geometric optimization of three-dimensional configurations of microchannels has not been performed yet.

Various research groups have been trying to model the real behaviour of thermal microfluidic chips. Governing equations of the two-phase flow/heat transfer problem have been developed through complicated mathematical models. Most of these equations have been solved numerically. However, proper finite element modelling has not been addressed to consider all details of the fluid flow/heat transfer phenomena.

In light of the above, the current thesis considers the geometric optimization of microchannels with a concentration on channel cross-sections and channels configurations, using the finite element method. In order to capture the intricate flow and heat transfer phenomena within a microchannel having a large aspect ratio (on the order of 1000), a compromise between cross-sectional nodal resolution and computational time must be found. A practical solution to account for both the flow in the microchannel and the effect of the microchannels configuration on the outer-channel heat transfer behaviour is to study each region separately (see Figure 2-8). Accordingly, the micro modelling deals with the coupled heat transfer-fluid flow problem inside the microchannel. This approach ignores the outer-channel phenomena and enables the optimization of the microchannel cross-section geometry to maximize the micro heat pipe performance. Then, the macro modelling considers the channels as a homogeneous material in order to investigate the effect of channels geometry on the thermal performance of the entire microfluidic chip. Later on, a threedimensional study is presented to obtain the best channel configurations.

Alongside with the geometric studies, finite element modelling was employed to replicate the experiment. After optimizing the microchannel cross-section, a 
single microchannel was investigated under two-phase flow conditions and the results were evaluated with the literature. The model was capable of capturing all details of the heat transfer/fluid flow phenomena and a good correlation with the literature was achieved. Subsequent to the three-dimensional optimization of microchannels, a finite element model was built in order to investigate the twophase flow inside the three-dimensional network of microchannels. The model was able to replicate the two-phase circulation of the working fluid inside the microchannels.

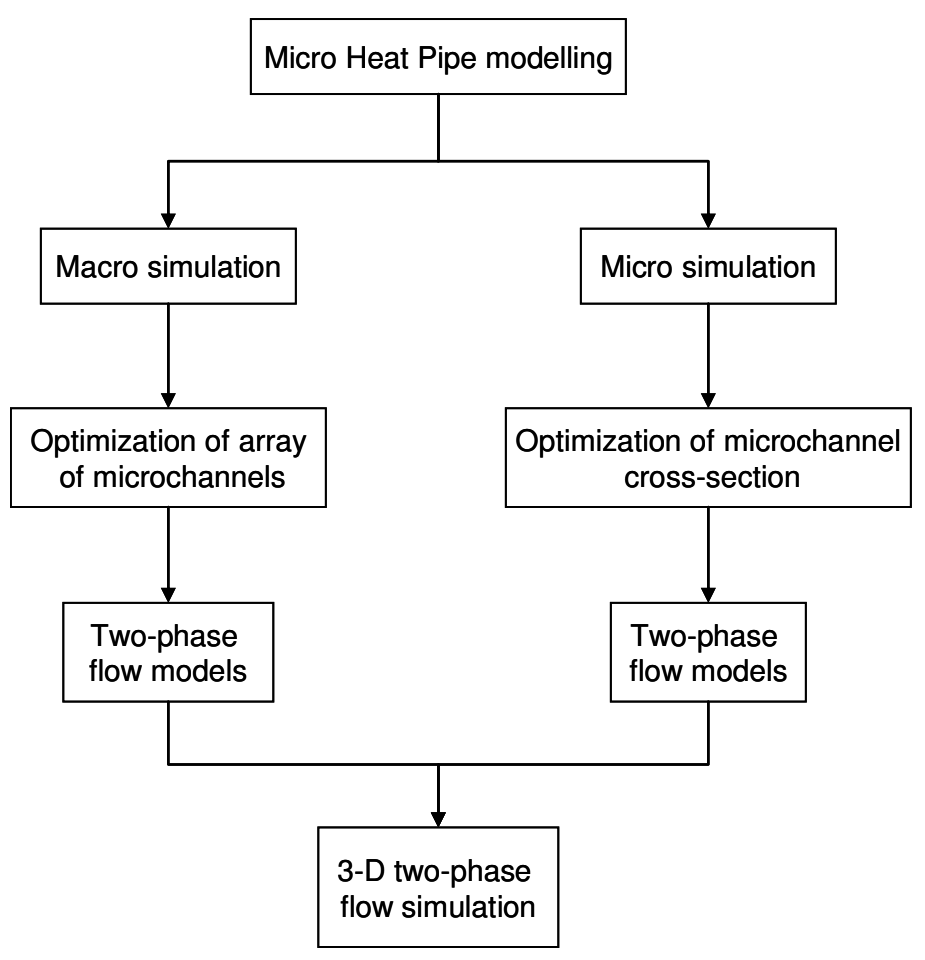

Figure 2-8: Micro Heat Pipe modelling flowchart for micro and macro simulations

During the entire investigation, direct-write method was assumed to be the manufacturing method for the microfluidic chip. Therefore, epoxy was selected as the surrounding material, and the three-dimensional optimization was accomplished based on the manufacturing feasibilities of direct-write technique. 


\section{Chapter 3 Micro modelling}

In order to capture the fluid flow/heat transfer phenomena inside the microfluidic chips, micro modelling investigations are required. A number of microscale

phenomena, e.g. dry-out length, have significant effects on the thermal performance of microfluidic devices. Thus, a complete understanding of the micro fluidic devices that leads to a prediction of their thermal performance depends on the microscale phenomena. This chapter presents three micro models of a single microchannel, where the interaction between the inner-channel and outer-channel phenomena is not considered.

- A preliminary model considering the microchannel as a homogeneous material with effective thermal conductivity is used to investigate the heat fluxes in the interfaces of evaporator, adiabatic section, and condenser.

- Single-phase models of a small section of the condenser employed to investigate the effect of microchannels' cross-section on the thermal performance of microfluidic chip.

- Two-phase models replicate the fluid flow/heat transfer behaviour of microfluidic chips and consider the effect of characteristic parameters (e.g. liquid filling ratio) on the behaviour of microfluidic chips.

The results of the micro modelling will be used for the macro modelling presented in chapter 4 .

\subsection{Preliminary model}

Thermal microfluidic chips operate under different boundary conditions depending on the application in which they are used. For single-phase 
microchannels, the circulation of the working fluid causes a relatively high thermal conductivity along the channels - compared to the surrounding epoxy which has a thermal conductivity in the order of $1 \mathrm{~W} / \mathrm{mK}$. An even higher thermal conductivity is achieved through the phase change for two-phase flows.

\subsubsection{Assumptions and settings}

The typical aspect ratio of microchannels (on the order of 1000) as well as their high effective conductivity causes a dominant one-dimensional heat flux along the axial direction of the microchannel. When the boundary heat flux is perpendicular to the microchannel axial direction, the heat flux turns in the axial direction in a reorientation zone between the evaporator (or condenser) and the adiabatic section. The investigation of the characteristics of this zone (e.g. length and heat flux in different directions) allows the simplification of the boundary conditions. For example, if the reorientation zone can be ignored, all heat fluxes applied to the microchannel can be assumed to be in the microchannel axial direction, and that causes a significant simplicity in the application of the boundary conditions for future analysis.

A finite element model of a single microchannel was defined in ANSYS 10.0. The microchannel was modeled as a homogeneous material with equivalent material properties obtained from the literature [2]. It was demonstrated that the microchannel working fluid content had an effect on the thermal performance. A liquid filling ratio of 20-25\% led to the best results [3]. Therefore, a liquid filling ratio of $22.5 \%$ by volume was used to derive the microchannel equivalent material properties. Table 3-1 lists the microchannel material properties used in the simulations. The density and specific heat for the microchannel were calculated based on the rule of mixtures with the properties of liquid water and vapour. Based on the results of the previous investigations [50] a thermal conductivity of $400 \mathrm{~W} / \mathrm{mK}$, which is close to that of copper, was selected for microchannels. 
Table 3-1: Effective material properties for the microchannel

\begin{tabular}{lc}
\hline Property & Microchannel $^{*}$ \\
\hline Density $\left(\mathrm{kg} / \mathrm{m}^{3}\right)$ & 225 \\
Specific heat $\left(\mathrm{J} / \mathrm{kg}^{\circ} \mathrm{C}\right)$ & 2500 \\
Thermal conductivity $\left(\mathrm{W} / \mathrm{m}^{\circ} \mathrm{C}\right)$ & 400 \\
\hline
\end{tabular}

* Effective properties based on a fluid content of $22.5 \%$ by volume.

The microchannel was represented for a two-dimensional model with a length of $15 \mathrm{~mm}$ and a thickness of $200 \mu \mathrm{m}$. Figure 3-1 shows a schematic of the finite element model. The microchannel was meshed with 24000 square elements each with 8 nodes and the model boundary conditions were applied according to Figure 3-1. The heat fluxes at the evaporator and the condenser sections were 15500 $\mathrm{W} / \mathrm{m}^{2}$ and $-15500 \mathrm{~W} / \mathrm{m}^{2}$, respectively. The hot source temperature, $T_{h}$, and the cold sink temperature, $T_{c}$, were set to $90^{\circ} \mathrm{C}$ and $10^{\circ} \mathrm{C}$, respectively. The applied boundary conditions were selected in accordance with the typical microchannels operating conditions. The low conductivity of the surrounding epoxy causes low heat transfer trough the adiabatic and the bottom surface in evaporator and condenser sections.

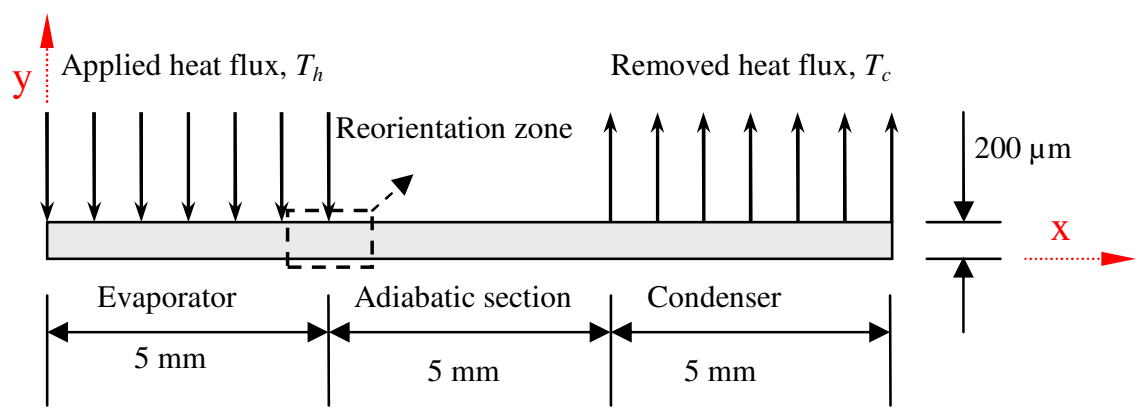

Figure 3-1: Model of a single microchannel 


\subsubsection{Results and discussion}

The reorientation zone can be defined as an area where the heat flux reorients from the applied direction (i.e. vertical) to the axial direction of microchannel. Figure 3-2 shows the iso-surfaces for the heat flux, where heat fluxes in $\mathrm{x}$ and $\mathrm{y}$ directions are shown in Figure 3-2-a and b, respectively. Figure 3-2-c replicates Figure 3-2-a (on the top) and Figure 3-2-b (on the bottom) with the concentration on the heat flux transient zone, reorientation zone. According to Figure 3-2, the length of the transition area - reorientation zone - is comparable with the thickness of the microchannel. Far from the reorientation zone, there is a uniform heat flux either in $\mathrm{x}$ or $\mathrm{y}$-direction.

Figure 3-3 shows the heat flux in the steady state conditions for the $\mathrm{x}$ and $\mathrm{y}$ direction for different positions along the thickness of the microchannel. The dimensionless length of the microchannel is obtained by dividing the axial position by the length of the microchannel. According to Figure 3-1, the length of microchannel was divided into three equal sections: evaporator, adiabatic section, and condenser. The adiabatic section of the microchannel, indicated in Figure 3-3, has a uniform heat flux far from the reorientation zone. As shown in Figure 3-3-a, the top line of the microchannel follows the applied boundary conditions, and there is a sudden peak in the beginning of adiabatic section. Towards the bottom line of the microchannel, the heat flux in the x-direction smoothly reaches the nominal value in the adiabatic section. The nominal values are determined by the heat flux and temperature boundary conditions in the evaporator and condenser. A comparison between the value of the singularity peak at the reorientation zone with adiabatic section and the nominal value of heat flux in the $\mathrm{x}$-direction is shown in Figure 3-3-a. 


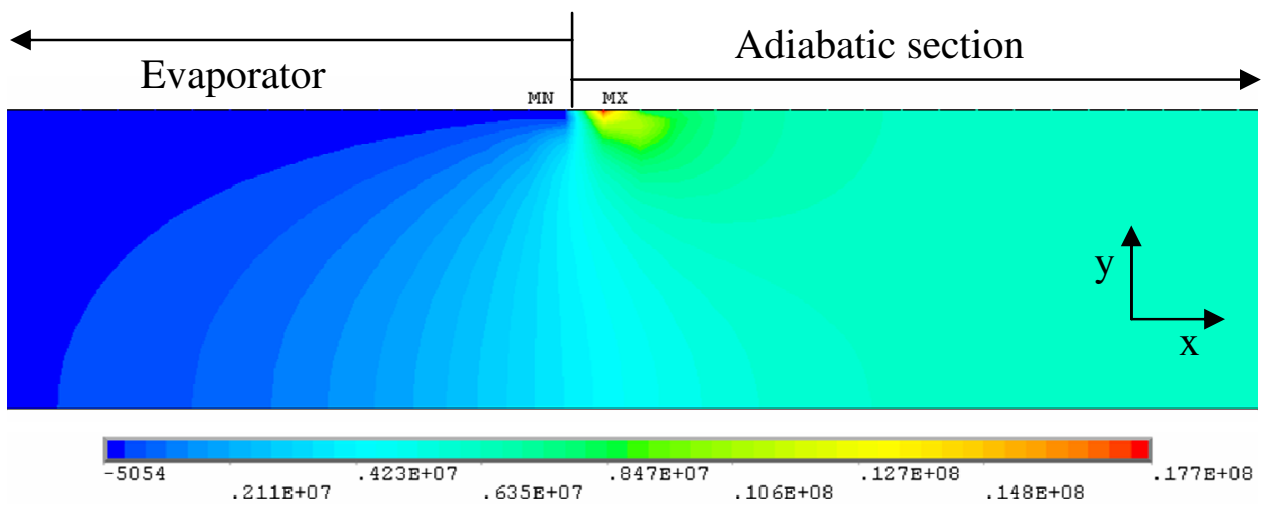

(a)

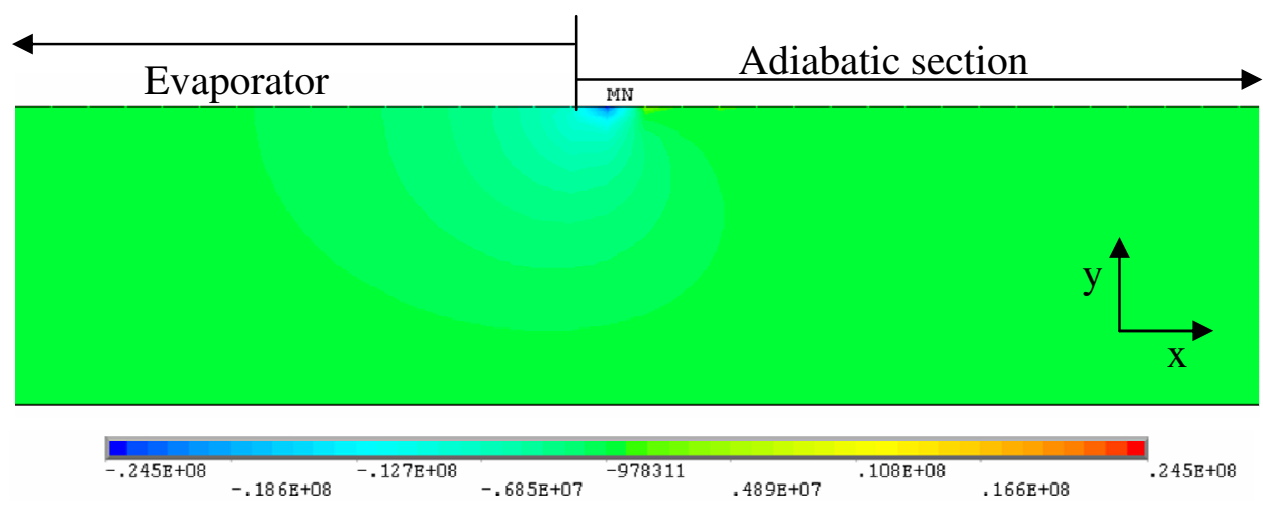

(b)

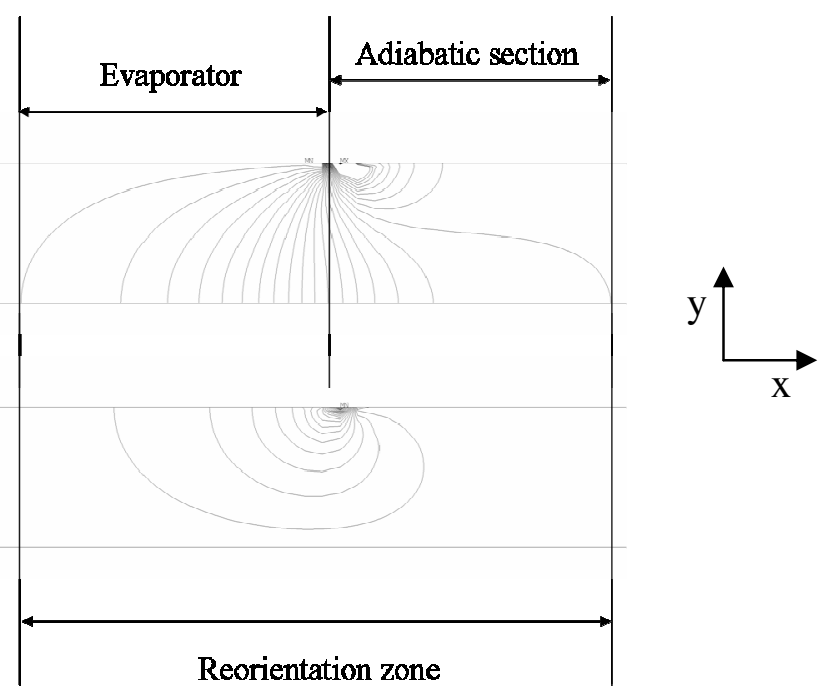

(c)

Figure 3-2: Iso-surfaces of heat flux $\left(\mathrm{W} / \mathrm{m}^{2}\right)$ in the reorientation region (see Figure 3-1), a) in the $\mathrm{x}$-direction, $\mathbf{b}$ ) in the $\mathrm{y}$-direction. $\mathbf{c})$ The length of the reorientation zone with the $\mathrm{x}$-direction heat flux (on the top) and y-direction heat flux (on the bottom). 
Similarly, Figure 3-3-b shows the y-direction heat flux in different positions of the microchannel. The singularity peak value at the reorientation zone decreases towards the bottom line of the microchannel. A comparison between the reorientation peaks and the nominal value of y-direction heat flux is also shown in Figure 3-3-b.

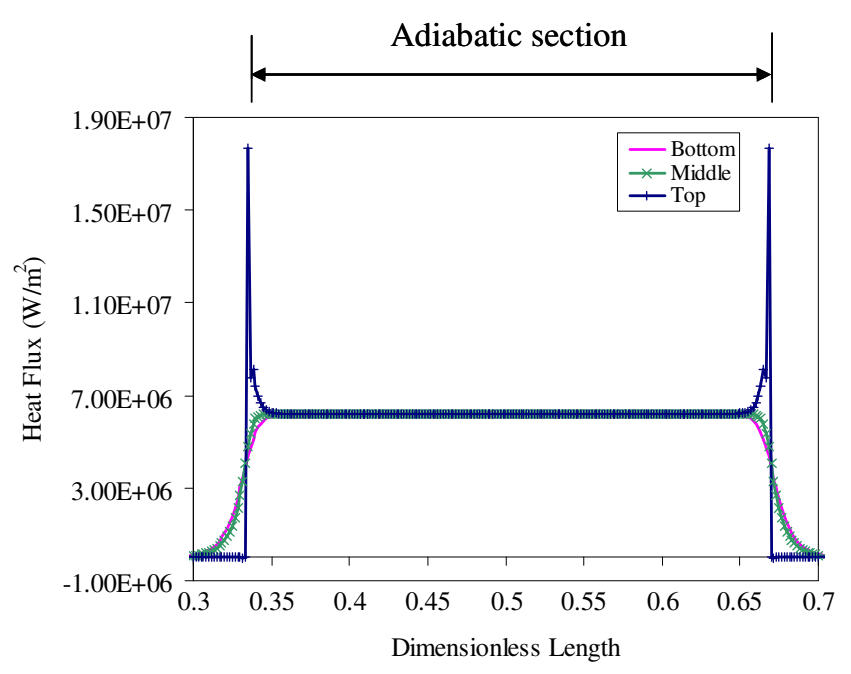

(a)

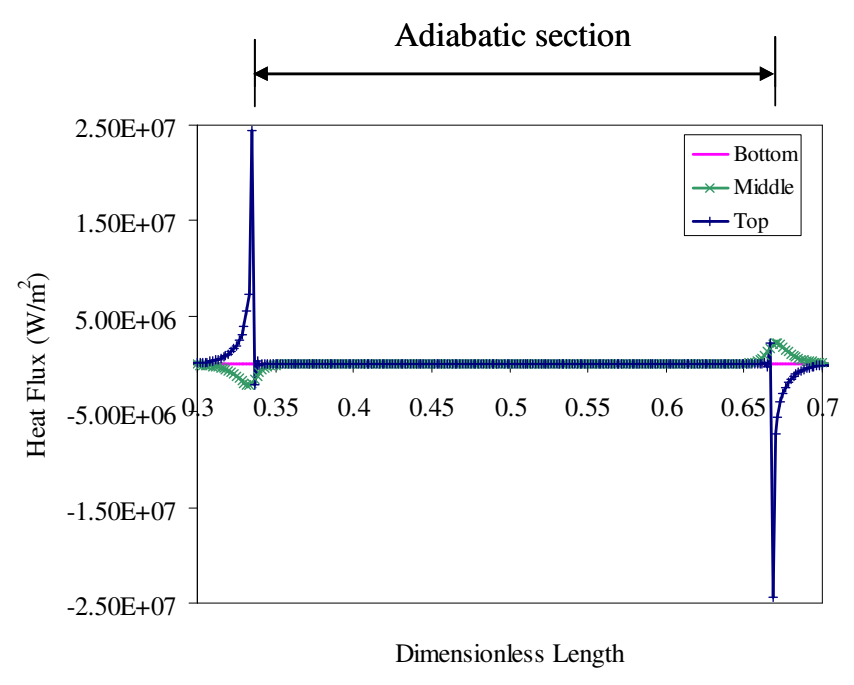

(b)

Figure 3-3: Heat flux from bottom $(y=0 \mu \mathrm{m})$, top $(\mathrm{y}=200 \mu \mathrm{m})$, and middle $(\mathrm{y}=100 \mu \mathrm{m})$ of the microchannel: a) x-direction b) y-direction 
Investigation on the reorientation zone under steady state conditions determines the heat transfer phenomena behaviour in this region. The results of Figure 3-3 suggest that considering the microchannel width (i.e. $200 \mu \mathrm{m}$ ), the length of the reorientation zone is less than three times the microchannel thickness. It was shown that far from the reorientation zone, the direction of applied heat flux (i.e. boundary conditions) has no effect on the overall thermal performance of microfluidic chip. Thus, regardless of the applications of microfluidic chip, the heat flux boundary conditions can be applied in the axial direction of microchannels and that will cause small errors in the reorientation zone.

\subsection{Single-phase model}

The first step in the geometric optimization of a planar microfluidic chip is the optimization of microchannel cross-section. The coupled heat transfer/fluid flow problem for a single-phase flow was modeled using ANSYS CFX-5.7.1. This finite element software solves the continuity (Equation 1), momentum (Equation 2) and energy (Equation 3) governing equations simultaneously.

$$
\dot{m}_{\text {in }}=\dot{m}_{\text {out }}
$$

where $\dot{m}_{\text {in }}$ is the inlet mass flow rate and $\dot{m}_{\text {out }}$ is the outlet mass flow rate.

$$
\rho \frac{D v}{D t}=\rho B-\nabla p+\mu \nabla^{2} v+F_{v i s}
$$

where $\frac{D}{D t}$ is the material derivative, $\rho$ is the fluid density, $v$ is the velocity, $t$ is the time, $B$ is the body force acting on the infinitesimal control volume, $p$ is the pressure, $\mu$ is the dynamic viscosity, and $F_{v i s}$ is the viscous force (the only surface force) applied to the infinitesimal control volume.

$$
\dot{E}_{\text {in }}+\dot{Q}_{\text {out }}-\dot{E}_{\text {out }}=\frac{\partial U_{l}}{\partial t}
$$


where $\dot{E}_{\text {in }}$ is the rate of energy that enters the control volume, $\dot{Q}_{\text {out }}$ is the rate of heat transfer that exits the control volume, $U_{l}$ is the internal energy of the fluid, and $\dot{E}_{\text {out }}$ is the rate of energy that exits the infinitesimal control volume. The terms $\dot{E}_{\text {in }}$ and $\dot{E}_{\text {out }}$ include the potential energy, kinetic energy and enthalpy.

\subsubsection{Assumptions and settings}

In order to compromise the computer run time and the nodal resolution of the model, a small section of the condenser was investigated. The analysis was performed over a length of $1 \mathrm{~mm}$ along the axis of the microchannel. Alongside the research on the evaporator section, the condenser section is also of importance especially for special microfluidic chips such as micro heat sinks. The similar nature of evaporator and condenser in single-phase microfluidic chips leads to the same geometric optimization results. In order to determine the optimum crosssection geometry, three-dimensional configurations of different cross-section geometries were modeled. Figure 3-4 shows the investigation section of the analysis as well as the respective dimensions of different cross-sections. The hydraulic diameters were $247.2 \mu \mathrm{m}, 400 \mu \mathrm{m}$ and $229.2 \mu \mathrm{m}$ for the triangular, circular and trapezoidal cross-section respectively. Water was selected as the working fluid and the effect of natural convection was studied. It was expected that the body force effects were negligible, thus the hydrostatic pressure gradient was ignored. A comparison between buoyant and non-buoyant models was accomplished and small effect of buoyancy was observed. Therefore, ignoring the buoyancy, a symmetric model of the microchannel was meshed with 1.5 million tetrahedron elements. A Reynolds number of 10 corresponding to the typical microchannels working conditions was used [28]. Steady state conditions were modelled for a single-phase flow and the effect of thermal radiation was neglected. No slip condition was applied to the microchannel walls. Figure 3-5 shows the schematic boundary conditions applied to the triangular cross-section. The inlet temperature $\left(T_{i}\right)$ was $293 \mathrm{~K}$ and the top heat flux $\left(\dot{Q}_{T}\right)$ was -15500 
$\mathrm{W} / \mathrm{m}^{2}$. Epoxy was chosen as the material for the microfluidic chip block, thus considering its low conductivity, a side heat flux $\left(\dot{Q}_{S}\right)$ of $-155 \mathrm{~W} / \mathrm{m}^{2}$ was applied to the model. These values were selected in accordance with the working condition of a typical thermal microfluidic chip.
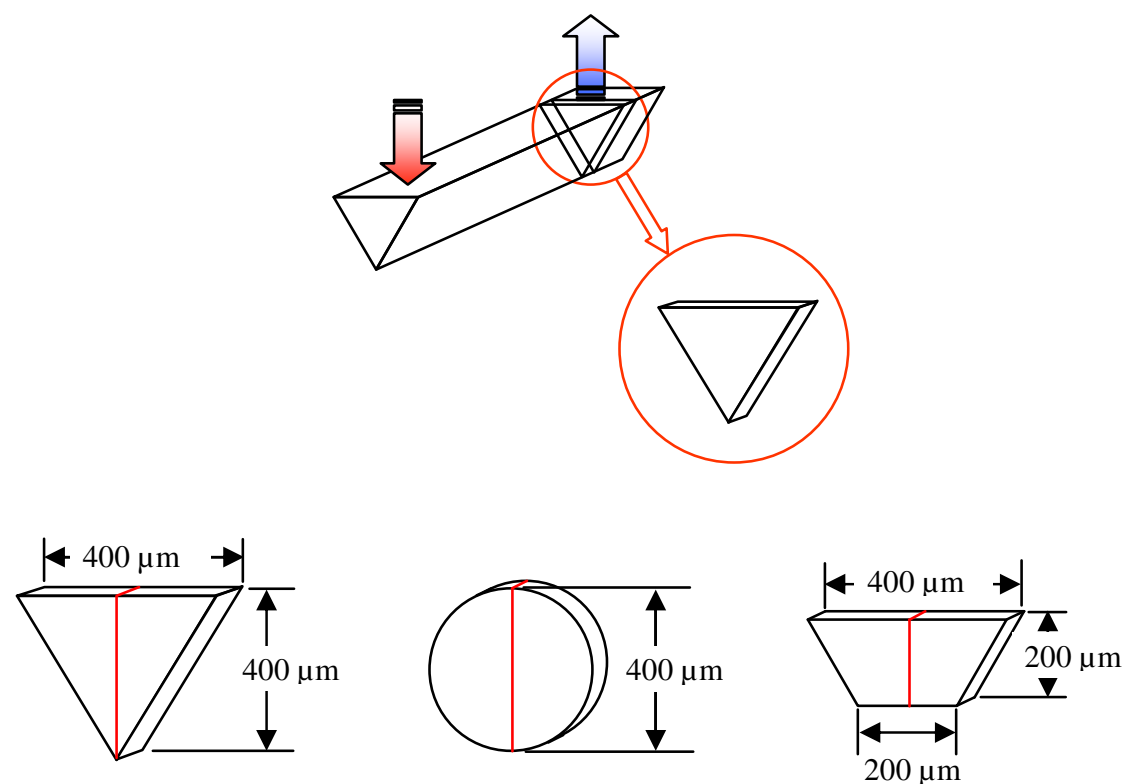

Figure 3-4: Modelling section, microchannel cross-section geometries and dimensions. The vertical line on the cross-section geometry corresponds to the model plane of symmetry.

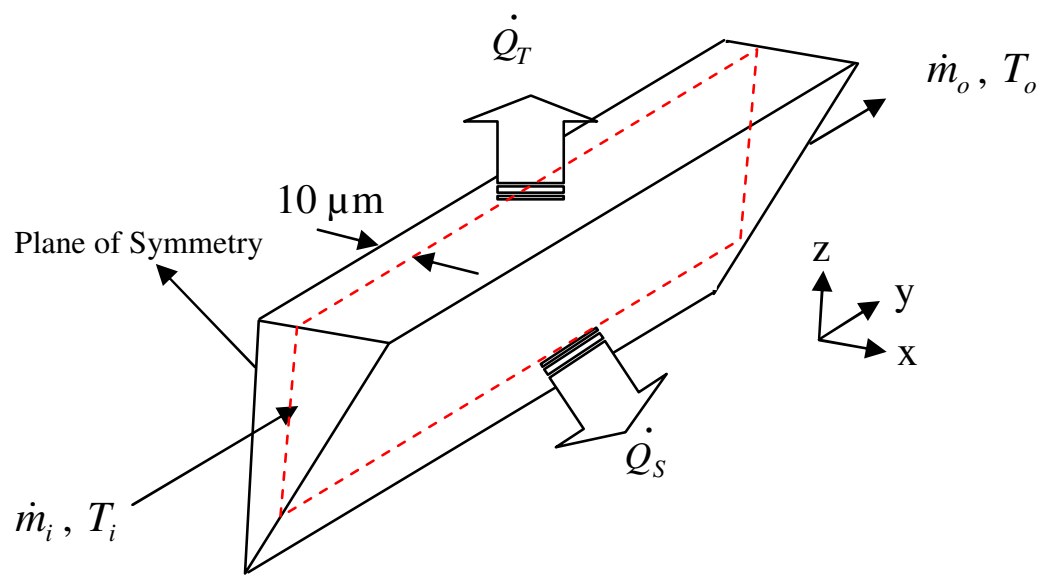

Figure 3-5: Typical boundary conditions applied to the microchannel; the triangular cross-section and the contour plane of Figure 3-6 shown (dashed line). 


\subsubsection{Results and discussion}

The microchannel geometries have different characteristics, e.g. various hydraulic diameters; therefore different flow/heat transfer behaviours were expected. Figure 3-6 shows the resultant velocity contours on a plane at a distance of $10 \mu \mathrm{m}$ from the plane of symmetry. The no slip boundary condition caused small disturbances to the fluid flow inside the channels that had no significant effects as observed from the uniform velocity profiles. Side wall effects at the bottom of the microchannel, where the symmetry and side planes coincide, were observed for the triangular cross-section (right edge of Figure 3-6-b). On the other hand, less disturbed flow was found for the trapezoidal cross-section (Figure 3-6-c), since the side plane is far enough from the plane of symmetry. For the circular crosssection (Figure 3-6-a), the effect of local disturbances appeared in the form of small vortices at the microchannel top and bottom edges. It should be mentioned that although the velocity gradient was very small (maximum $\pm 0.08 \%$ for the circular microchannel), the results could provide useful information on the formation of the velocity profile.

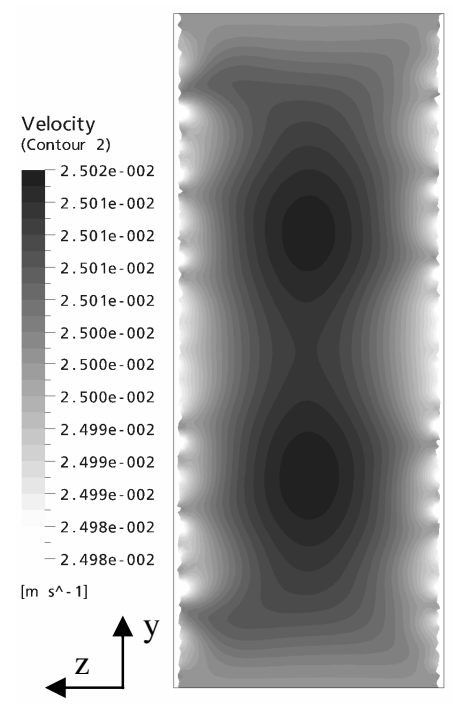

(a)

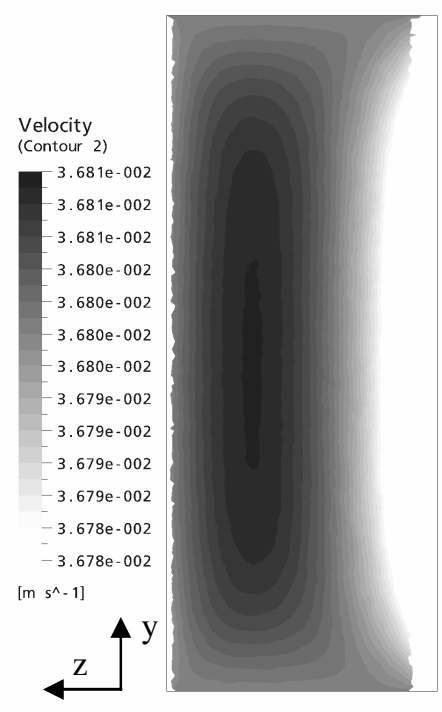

(b)

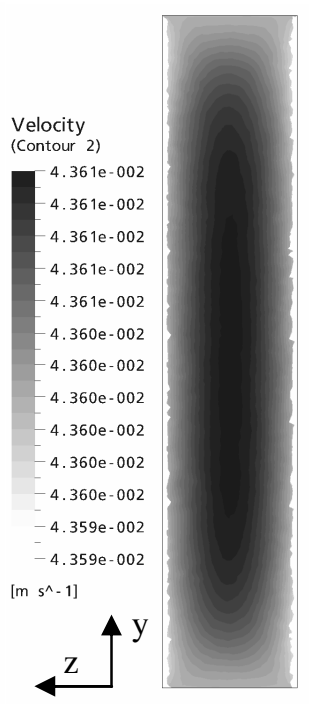

(c)

Figure 3-6: Steady state resultant velocity $(\mathrm{m} / \mathrm{s})$ contours at a distance of $10 \mu \mathrm{m}$ from the plane of symmetry (see Figure 3-5), the left side corresponds to the top of the microchannel for a) circular,

b) triangular, and c) trapezoidal cross-section. 


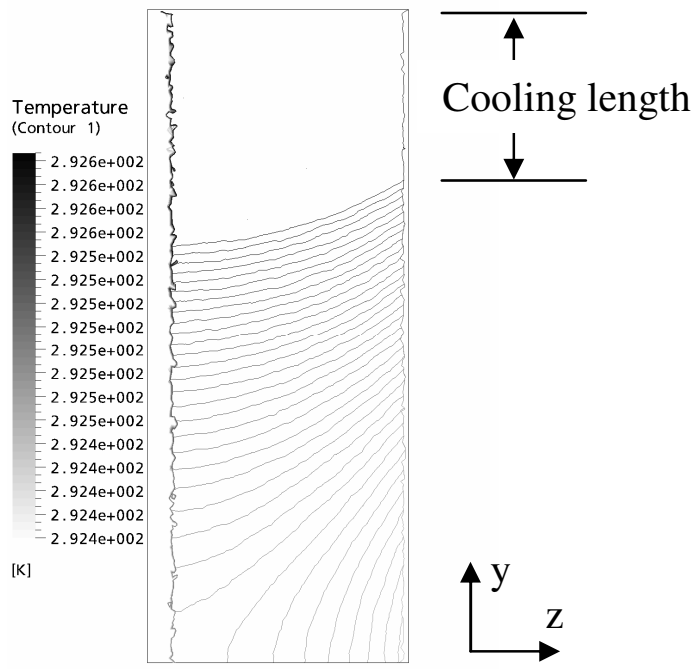

(a)

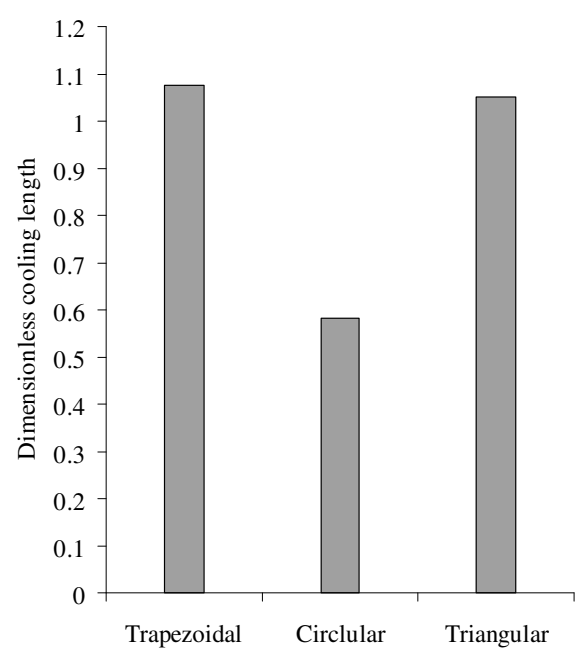

(b)

Figure 3-7: a) Steady state temperature (K) profile and cooling length for the triangular crosssection on a plane at a distance of $10 \mu \mathrm{m}$ from the plane of symmetry (see Figure 3-5). b)

Dimensionless cooling length for different microchannel cross-sections.

Figure 3-7 shows the temperature contours on a plane at a distance of $10 \mu \mathrm{m}$ from the plane of symmetry (see Figure 3-5) for the triangular cross-section with temperatures ranging from $292.4 \mathrm{~K}$ to $292.6 \mathrm{~K}$. To compare the effect of different microchannel geometries, a cooling length was defined as the distance from the inlet to a point corresponding to an arbitrary temperature gradient of $-4 \mathrm{E}-3 \mathrm{~K}$ from the inlet temperature. The cooling length for the triangular cross-section is shown in Figure 3-7-a. The cooling length obtained for the different cross-section geometries was divided by the corresponding hydraulic diameter (Figure 3-7-b). The circular cross-section had the lowest dimensionless cooling length compared to the triangular and trapezoidal cross-sections. This result suggests that generally, for microfluidic chips, the circular cross-section has a better cooling efficiency compared to the other geometries. However particularly, for microfluidic chips without micro pump, the absence of sharp edges for the circular cross-section significantly reduces the capillary pressure gradient necessary to provide flow inside the microchannel. To address the capillary pressure, an extensive investigation about the hydrophobicity of epoxy resin was conducted by Motoyama and Hackam [63]. They demonstrated that the contact angle of the 
surface of epoxy resin in the air at room temperature was $101 \pm 8^{\circ}$. Consequently, the outer-channel approach presented in the next chapter focuses only on the triangular, rectangular and trapezoidal cross-section geometry.

\subsection{Two-phase model}

This section presents the effect of sharp corners in a triangular cross-section to consider the thermal performance of micro heat pipes - as passive capillary pumped systems. The two-phase fluid flow helps to understand micro phenomena (e.g. phase change and capillary pressure) happening within the microchannels. Considering the common equilateral triangle, the importance of sharp corners in transferring liquid from the condenser to the evaporator is investigated.

\subsubsection{Assumptions and settings}

ANSYS CFX-5.7.1 finite element software was used to simulate the two-phase flow with heat and mass transfer in the microchannel. This software simulates the phase change phenomenon in the microchannel to properly replicate the coupled fluid flow/heat transfer problems. Since in the current two-phase analysis, the exact behaviour of the system (even in the reorientation zone) is interested, the assumption of applying axial heat boundary conditions - as it was proved early in this chapter - is not employed. The body forces were neglected, and hence considering the symmetry, half of the equilateral triangular microchannel with $200 \mu \mathrm{m}$ sides was modeled. Figure 3-8 shows the model and the dimensions. The $2 \mathrm{~cm}$ length of the channel was divided into three equal sections of $0.667 \mathrm{~cm}$ : evaporator, adiabatic section, and condenser. In the evaporator section, the heat was applied to the system from the top section, while in the condenser the heat was removed from the microchannel top section. The other boundaries of the microchannel were assumed to be adiabatic. The plane of symmetry, ABDC, was used to represent the simulation results. The line EE' at a distance of $75 \mu \mathrm{m}$ from the top line, $\mathrm{AB}$, was used to monitor the behaviour of the vapour phase, along the 
microchannel. The behaviour of the two-phase flow close to the corners was monitored along the line FF' at a distance of $3.2 \mu \mathrm{m}$ from the bottom line CD.

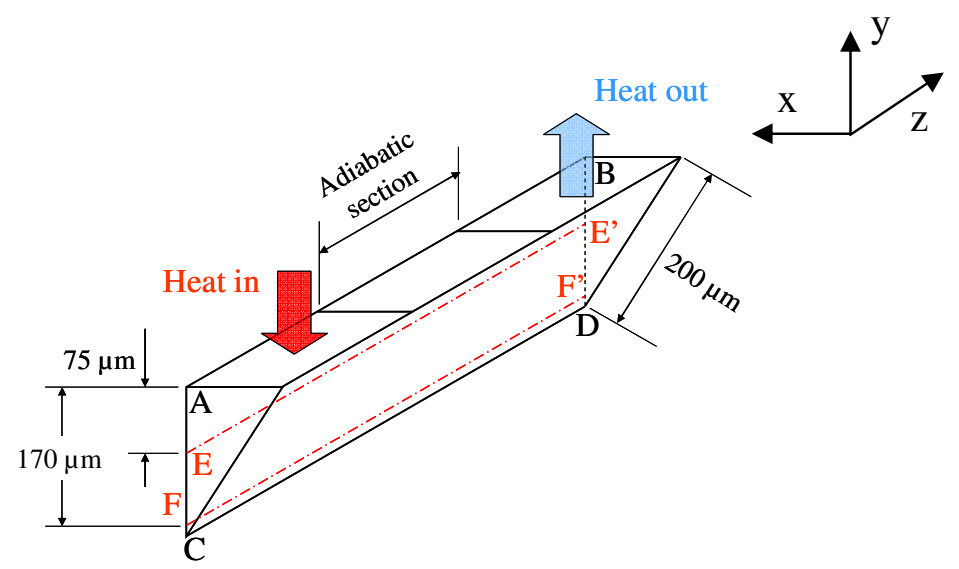

Figure 3-8: Geometry and boundary conditions of the symmetric model for two-phase analysis of a single micro heat pipe

The entire model was meshed with around 560000 tetrahedron elements. The size of elements was in the order of $10 \mu \mathrm{m}$. This number of elements presented a good nodal resolution in the axial direction of the microchannel. However, a denser mesh leads to a higher nodal resolution in the cross-sections - and of course longer run time. With this number of elements, each element had an average volume of around $310(\mu \mathrm{m})^{3}$, that indicated the minimum volume of the working fluid in the model.

Table 3-2 shows the various combinations of analysis parameters. Two types of thermal boundary conditions were applied to the model: heat flux boundary conditions, and temperature boundary conditions. Moreover, different filling ratios of water, as the working fluid, were investigated in the analysis. The analyses were examined after 2 and 30 seconds from the start-up, to consider the effect of time on the results. An initial temperature of $22^{\circ} \mathrm{C}$ was applied to the entire system, and the surface tension coefficient was set to $0.073 \mathrm{~N} / \mathrm{m}$. According 
to the software instruction, no slip boundary condition was applied for the liquid phase, whereas slip boundary condition was used for the vapour phase.

Table 3-2: Simulation definition matrix

\begin{tabular}{|c|c|c|c|c|c|}
\hline \multirow{2}{*}{ Run } & \multirow{2}{*}{ Filling ratio $(\%)$} & \multicolumn{3}{|c|}{ Boundary condition } & \multirow{2}{*}{ Time (s) } \\
\hline & & Type & Hot & Cold & \\
\hline 1 & 25 & Heat flux $\left(\mathrm{kW} / \mathrm{m}^{2}\right)$ & +0.1 & -0.1 & 2 \\
\hline 2 & 75 & Heat flux $\left(\mathrm{kW} / \mathrm{m}^{2}\right)$ & +0.1 & -0.1 & 2 \\
\hline 3 & 75 & Heat flux $\left(\mathrm{kW} / \mathrm{m}^{2}\right)$ & +1 & -1 & 2 \\
\hline 4 & 75 & Heat flux $\left(\mathrm{kW} / \mathrm{m}^{2}\right)$ & +5 & -5 & 2 \\
\hline 5 & 25 & Heat flux $\left(\mathrm{kW} / \mathrm{m}^{2}\right)$ & +10 & -10 & 2 \\
\hline 6 & 35 & Heat flux $\left(\mathrm{kW} / \mathrm{m}^{2}\right)$ & +10 & -10 & 2 \\
\hline 7 & 50 & Heat flux $\left(\mathrm{kW} / \mathrm{m}^{2}\right)$ & +10 & -10 & 2 \\
\hline 8 & 65 & Heat flux $\left(\mathrm{kW} / \mathrm{m}^{2}\right)$ & +10 & -10 & 2 \\
\hline 9 & 75 & Heat flux $\left(\mathrm{kW} / \mathrm{m}^{2}\right)$ & +10 & -10 & 30 \\
\hline 10 & 75 & Temperature $\left({ }^{\circ} \mathrm{C}\right)$ & 90 & 5 & 30 \\
\hline 11 & 75 & Temperature $\left({ }^{\circ} \mathrm{C}\right)$ & 70 & 30 & 2 \\
\hline 12 & 75 & Temperature $\left({ }^{\circ} \mathrm{C}\right)$ & 50 & 40 & 2 \\
\hline
\end{tabular}

\subsubsection{Model convergence}

The complex nature of the problem caused the convergence of the simulation to be challenging. The high instability of the system led to high fluctuations in the Root Mean Square (RMS) of the governing equations results. Figure 3-9 presents a typical graph of converging RMS's versus the number of iterations. The graph presents the convergence of the governing equations; for example the momentum in y-direction for water (RMS V-Mom Water).

According to Figure 3-9, all RMS's converged to 1E-3 after almost 100 iterations. However, the convergence rate was very slow after that. A compromise between the computer run time and the accuracy of the results was considered. A 
maximum number of 200 iterations, along with the residual target of $1 \mathrm{E}-4$, led to reasonable run times and accurate results. Thus, there were two criteria that defined the end of simulation run and each of these criteria that was reached first led the simulation to stop: firstly, if the number of iterations reached 200; and secondly, if all the RMS's placed bellow the target residual of 1E-4.

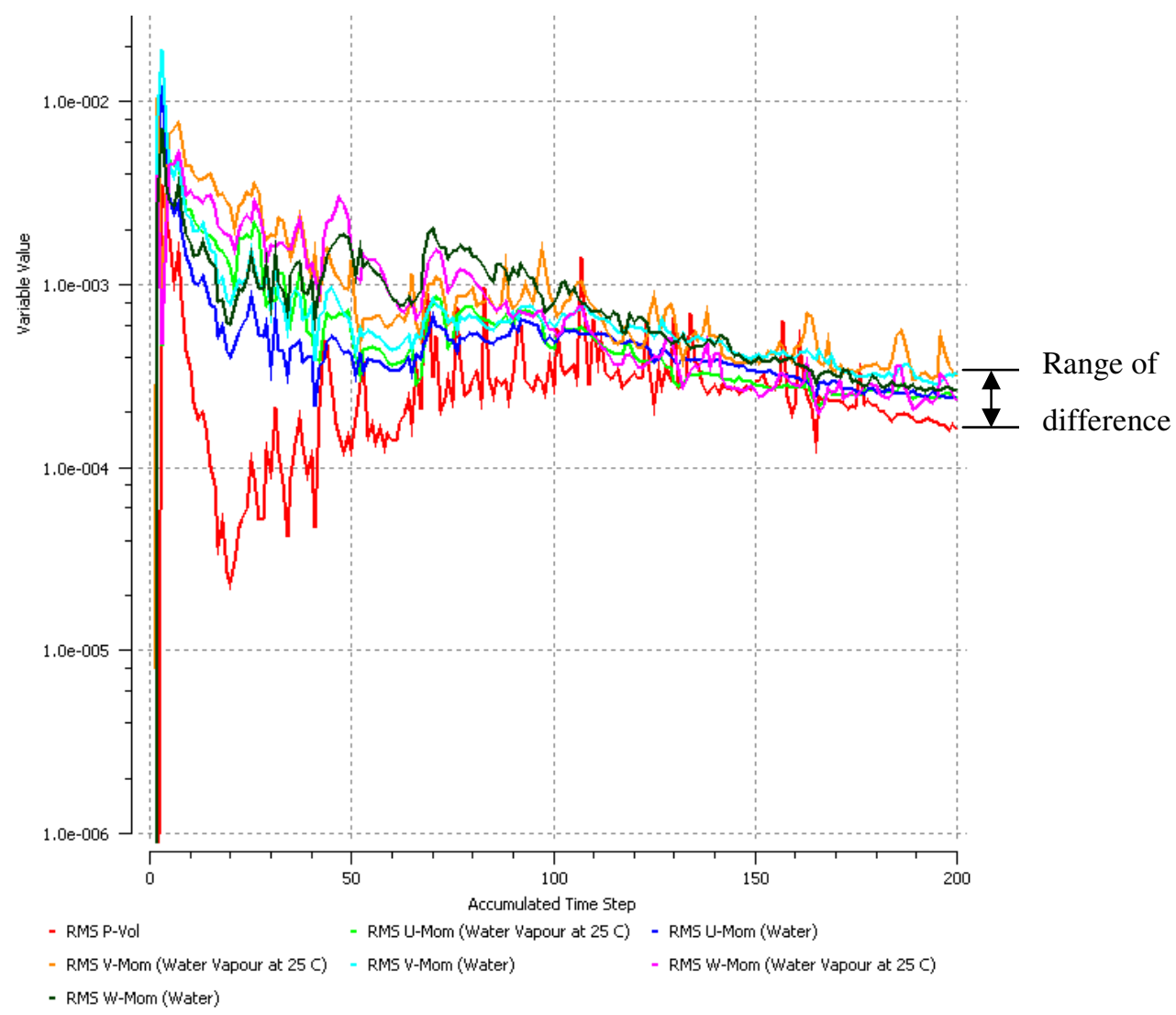

Figure 3-9: Simulation convergence for the Run 10, with the range of difference for different RMS's

Figure 3-10 shows the average RMS for different analyses. In order to obtain the average RMS for each of the analysis, the range of difference was divided by two. The deviations of the RMS's from the average value are also presented in Figure 3-10. The red line indicates the target residual of 1E-4. It is evident that for none of the simulations, all RMS's placed bellow the residual target. However, the final RMS's are very close to the residual target after 200 iterations (run time of around 8 hours on a dual $3.2 \mathrm{GHz}$ personal computer). 


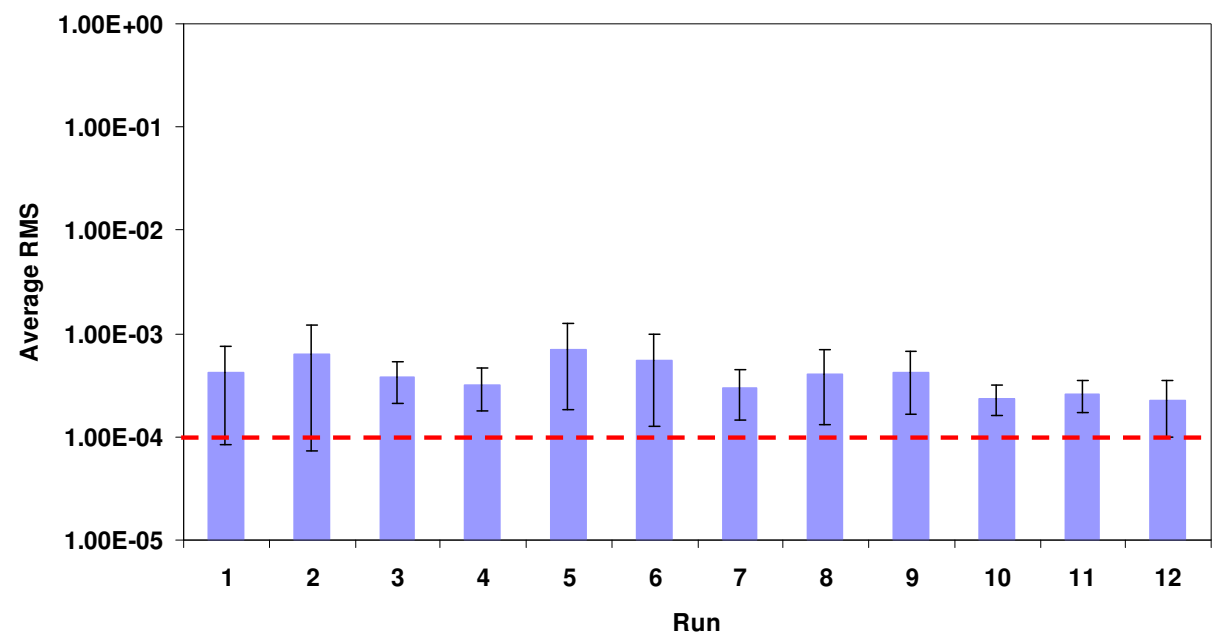

Figure 3-10: Average RMS for different analyses. The red line indicates the target RMS of 1E-4

\subsubsection{Results and discussion}

According to Table 3-2, various analyses were carried out and the simulations were converged regardless of the boundary conditions and the simulation settings. However, comparing the final results, it was found that severe boundary conditions (e.g. high heat flux or temperature difference) led to some instability in the final results. For example, the vapour conservative volume fraction for the analyses No. 11 and 12 are shown in Figure 3-11. The temperature difference in the evaporator and the condenser for the Run 12 is less than the Run 11. Therefore, less instability is visible for the Run 12. More fluctuation in the results can be noticed in the evaporator section $(0 \leq z \leq 0.0067 \mathrm{~m})$.

In this section, the capabilities and the challenges of the finite element software ANSYS CFX-5.7.1 are not investigated. Instead, some of the results of the simulation matrix are presented in order to investigate the fluid flow/heat transfer behaviour of the system, by replicating the experimental conditions for a twophase flow. 
Figure 3-12 presents the water (liquid phase) volume fraction in the evaporator section for Run 2. The bottom side of the contour demonstrates the sharp corner of the channel, and as it was expected, the volume fraction of the liquid water is almost unity in the corner. The presence of liquid water in the evaporator - the high temperature section - guaranteed the proper operation of the micro heat pipe. Applying high values of heat flux in the evaporator, may lead to a dry-out phenomenon, and reduce the thermal efficiency of the micro heat pipe, dramatically. The spectrum of colors between blue (volume fraction of zero) and red (volume fraction of unity) presents the evaporation process.

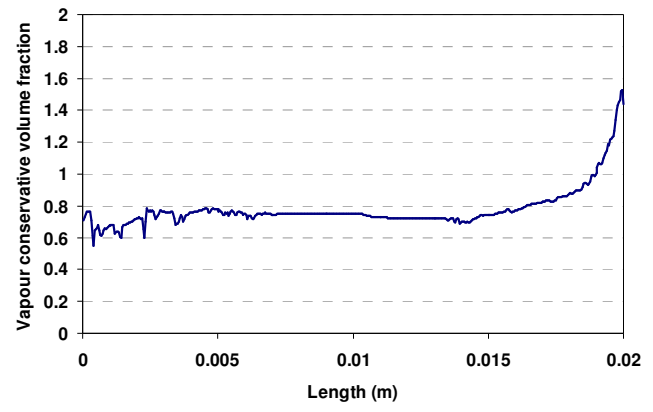

(a)

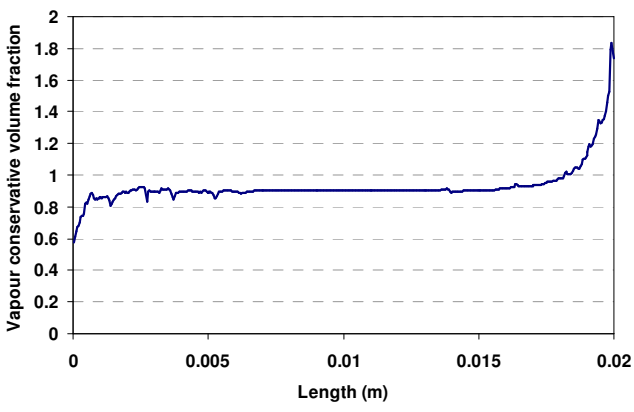

(b)

Figure 3-11: Vapour conservative volume fraction for a) Run 11, and b) Run 12

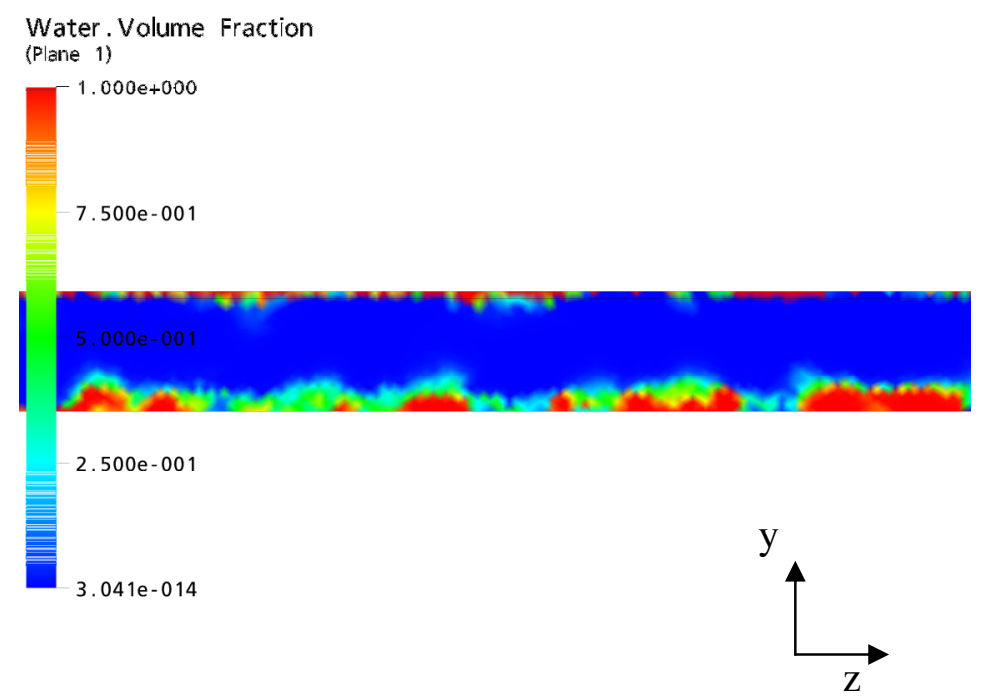

Figure 3-12: Water volume fraction in the evaporator for the Run 2.in the plane of symmetry. 
A better understanding of the phase change phenomenon can be observed in Figure 3-13, with the water/water vapour interphase mass transfer rate contours. The amount of interphase mass transfer rate indicates the rate of phase change. The latent heat in the phase change is directly related to the amount of heat transfer capacity.

As mentioned before, the phase change phenomenon mostly takes place in the interface of the liquid and vapour phases. Figure 3-13-a indicates the phase change phenomenon within the different sections of micro heat pipe, including evaporator, adiabatic section, and condenser. However, in Figure 3-13-b the phase change happening in the interface of different sections is presented. The top contour in Figure 3-13-b shows the interface of evaporator and the adiabatic section, while the bottom contour indicated the interface of the adiabatic section with the condenser. According to Figure 3-13-a, the maximum amount of phase change takes place in the condenser. The white area, surrounded by red lines, presents the area with the maximum phase change rate. Although there is phase change happening in the adiabatic section, the condenser and evaporator sections demonstrate high amounts of phase change. Figure 3-13-a shows that in the evaporator section - the top picture - the liquid exists only at the corners, and in the top line of the contour, which corresponds to the axis $A B$ in Figure 3-8, there is no film of liquid. However, in the adiabatic and condenser sections, the top line of the contour also participates in the phase change phenomena. It means that in these sections, liquid film covers the entire surface of the microchannel.

Figure 3-13-b considers the major phase changes within the interface between different sections, e.g. condenser and adiabatic sections (bottom contour). In the adiabatic section, the phase change phenomenon was negligible; however, the beginnings of condenser and evaporator corresponded to high values of phase change. The top contour in Figure 3-13-b shows the evaporator section. It is evident that the liquid enters the evaporator in the sharp corners, and there is significant amount of vaporization in the beginning of the evaporator section. 
Since the liquid flows in the corners, the evaporation process starts at the corners. On the other hand, the condensation process takes place in the beginning of the condenser (the bottom contour in Figure 3-13-b), and since the vapour flows in the middle of the channel, there are high amounts of condensation in the middle of the channel.

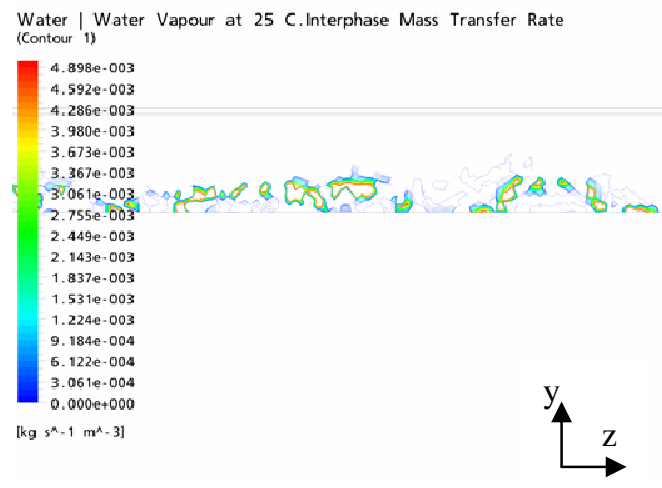

Water I Water Vapour at $25 \mathrm{C}$. Interphase Mass Transfer Rate
(Contour 1 )
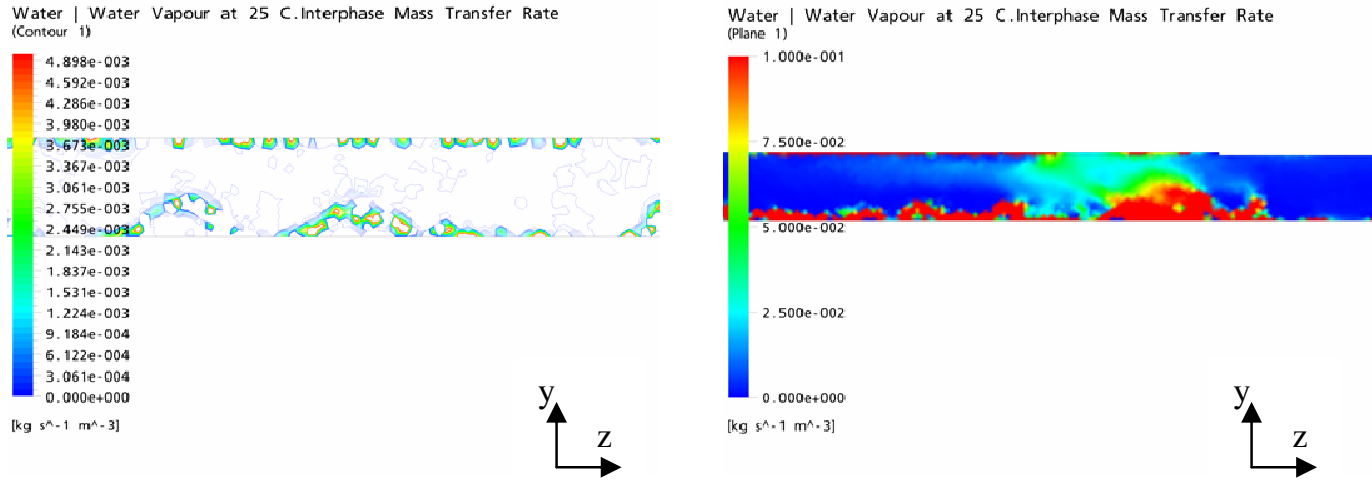

Water I Water Vapour at $25 \mathrm{C}$. Interphase Mass Transfer Rate

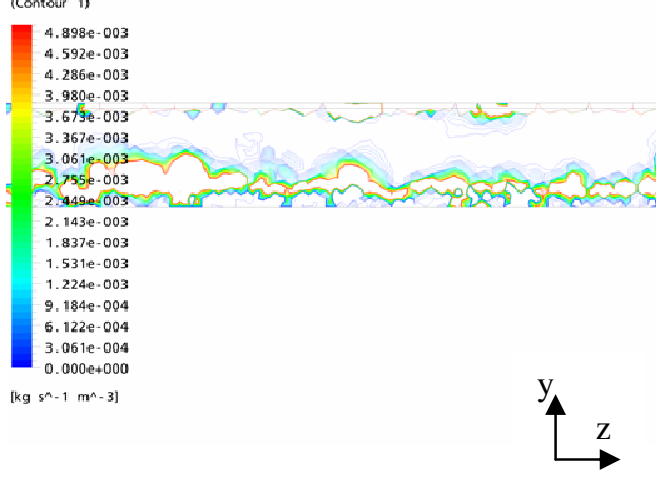
Water I Water Vapour at 25 S.Interphase Mass Transfer Rate
(Plane 1)

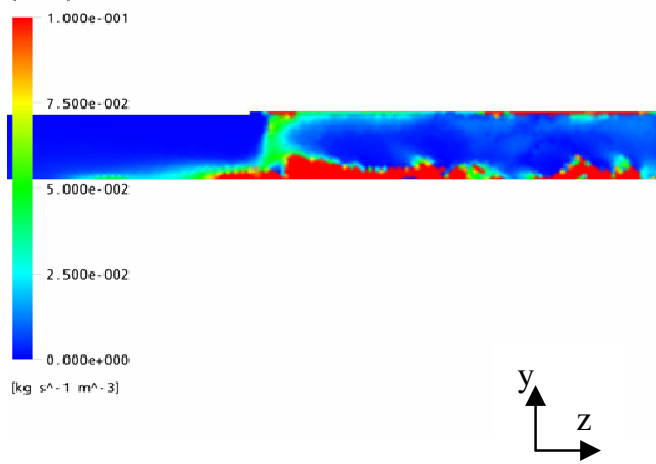

(a)

(b)

Figure 3-13: Water/water vapour interphase mass transfer rate $\left(\mathrm{kg} / \mathrm{m}^{3} \mathrm{~s}\right)$ in the plane of symmetry

a) for the Run 1, and b) for the Run 9. The top figures show the evaporator, the middle figure demonstrates the adiabatic section, and the bottom ones indicate the condenser. 
Besides the numerical and analytical modelling, experimental investigations [64] were carried out in the project to prove the viability of micro heat pipes manufactured by direct write method. Triangular microchannels with different dimensions were manufactured by depositing different layers of a fugitive ink. The filling and sealing process were performed, successfully. Thus, the border between the two phases was distinguishable. Figure 3-14 shows the interface between the two phases, captured in the experimental investigations phase. The agreement between Figure 3-14 and Figure 3-13-a illustrates the validity of the numerical simulations. It also demonstrates the ability of direct write method for the manufacturing of micro heat pipes.
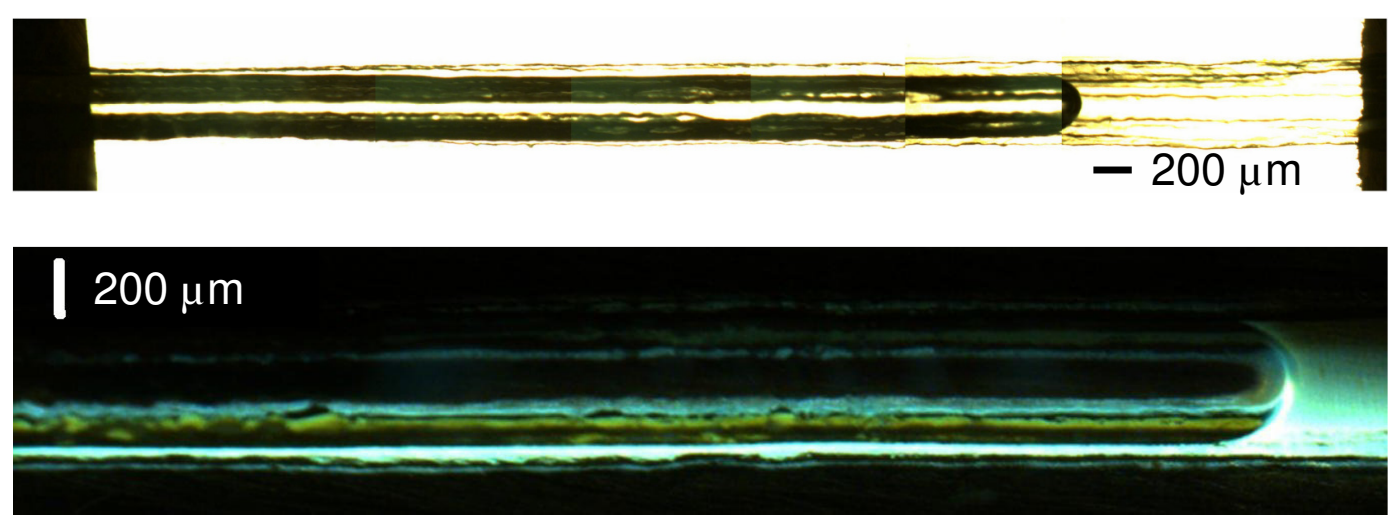

Figure 3-14: Liquid/gas interface in the experimental investigations [64]

Water vapour temperature and shear strain rate contours are shown in Figure 3-15. According to Figure 3-15-a the temperature gradient in the adiabatic section is negligible. Figure 3-15-b shows the rate of shear strain in the vapour flow. As mentioned before, in an operating micro heat pipe, the liquid and vapour phases flow in opposite directions. Thus, there are shear effects in the border of these two phases. Consequently, the shear strain rate can also be an index to recognize the border between liquid and vapour phases. The distinguishable interface between the two phases in Figure 3-15 is in accordance with the previous results of the volume fraction. The high shear strain rate indicates the zone where the two phases are mixing. 


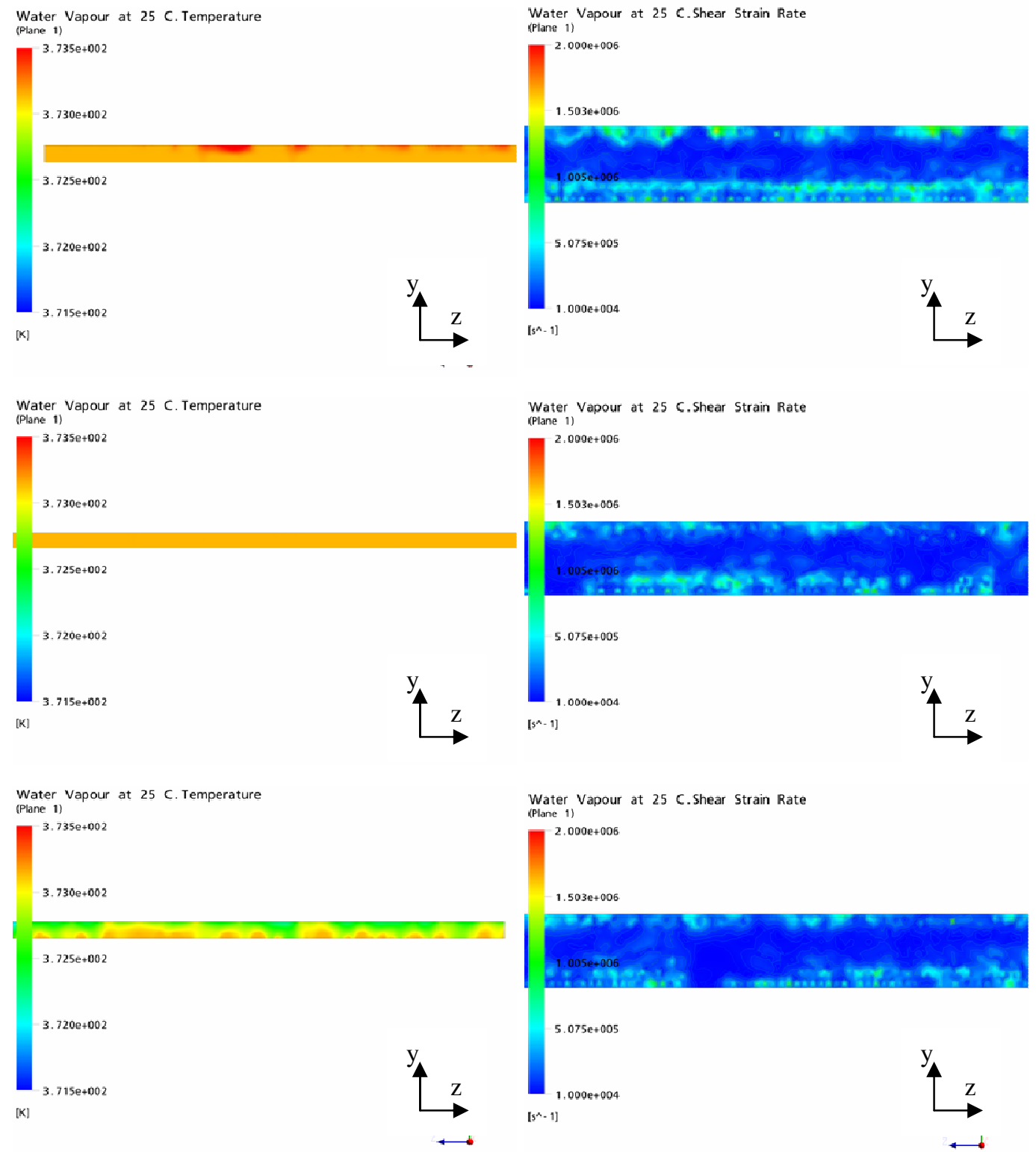

(a)

(b)

Figure 3-15: a) Water vapour temperature (K) in the plane of symmetry for the Run 2, b) Water vapour shear strain rate $(1 / \mathrm{s})$ in the plane of symmetry for the Run 1 . The top figures show the evaporator, the middle figures demonstrate the adiabatic section, and the bottom ones indicate the condenser.

Water temperature in the plane of symmetry for the Run 1 is shown in Figure 3-16. It is obvious that water had almost the same temperature all over the microchannel, except at the top surfaces of the evaporator and condenser, where 
the heat flux boundary conditions were applied. The very small temperature gradient during the length of the microchannel indicated the high effective thermal conductivity of the microchannel. The effective thermal conductivity of the microchannel, $k_{\text {eff }}$, is calculated as follows [3]

$$
k_{\text {eff }}=\frac{\dot{Q}_{m}}{\left(T_{\text {evap }}-T_{\text {cond }}\right) / L_{\text {eff }}}
$$

where

$$
L_{\text {eff }}=L_{\text {adiab }}+\frac{1}{2}\left(L_{\text {evap }}+L_{\text {cond }}\right)
$$

and $\dot{Q}_{m}$ is the heat flux in the microchannel, $T_{\text {evap }}$ is the evaporator temperature, $T_{\text {cond }}$ is the condenser temperature, $L_{\text {eff }}$ is the effective length of the microchannel, $L_{\text {adiab }}$ is the length of the adiabatic section, $L_{\text {evap }}$ is the evaporator length, and $L_{\text {cond }}$ is the condenser length.

Based on the results of Run 1, the effective thermal conductivity of the microchannel was estimated as $3333.3 \mathrm{~W} / \mathrm{mK}$, which is almost 8 times higher than that of copper. It should be mentioned that this effective thermal conductivity was calculated based on the water temperature difference in the evaporator and the condenser. The temperature difference for the liquid phase (Figure 3-16) was much less than the vapour phase (Figure 3-15-a) - i.e. 0.0004K for the liquid phase and $0.6 \mathrm{~K}$ for the vapour phase. Therefore, using the vapour temperature difference (in case of dry-out phenomenon) in the calculation of the effective thermal conductivity would dramatically reduce the thermal conductivity to 2.2 $\mathrm{W} / \mathrm{mK}$. It is evident that with the increase of heat flux in the evaporator, liquid water in the evaporator is replaced with the vapour with higher temperature and the effective thermal conductivity of the microchannel decreases. Thus, for higher heat fluxes, less effective thermal conductivity is expected. 


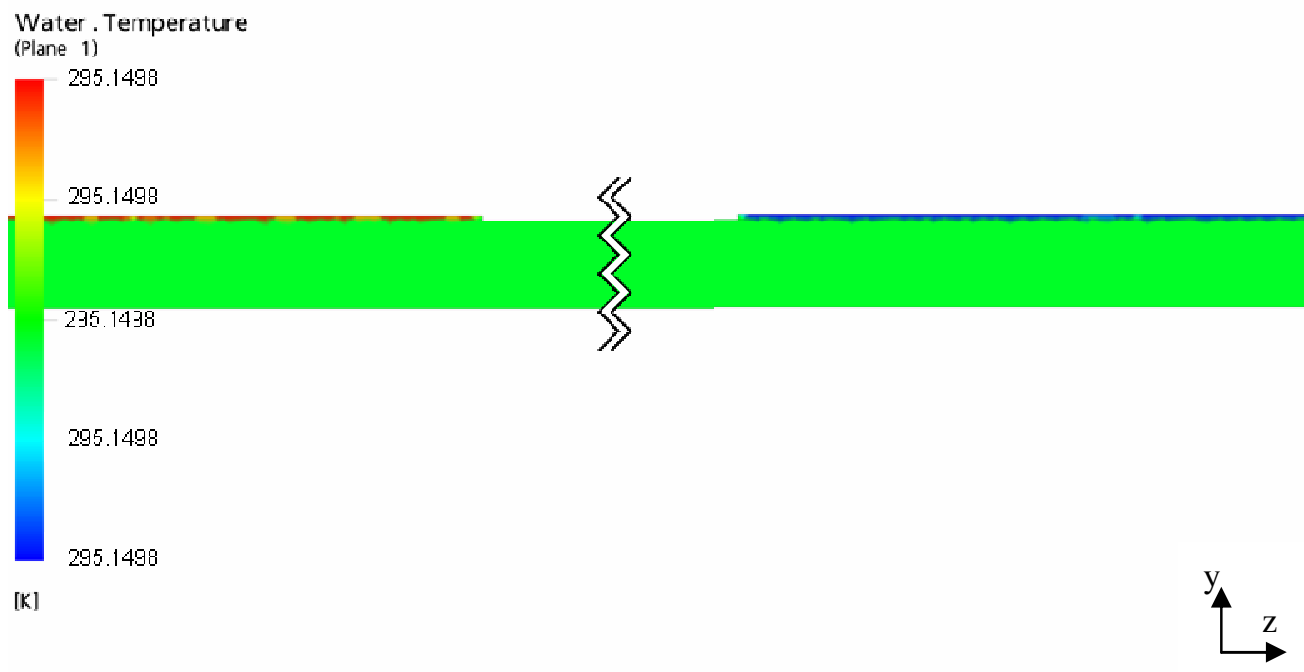

Figure 3-16: Water temperature $(\mathrm{K})$ in the plane of symmetry for the Run 1

Research has been conducted in the literature to measure the effective thermal conductivity of microfluidic chips. Duncan and Peterson [50], [42] achieved effective thermal conductivity in the range of $250-300 \mathrm{~W} / \mathrm{mK}$. However, it should be considered that the effective thermal conductivity of a microfluidic chip is a combination of effective thermal conductivities of the microchannel and the surrounding material. Based on the microchannels dimension [47] and the rule of mixture, an effective thermal conductivity of $3158.87 \mathrm{~W} / \mathrm{mK}$ was calculated for microchannels. The difference between the effective thermal conductivities obtained from the model and the literature is $5.5 \%$. The correlation between the results demonstrates the validity of the model.

Figure 3-17 presents the pressure along the line EE'. Based on the model, the length of the channel, which was $2 \mathrm{~cm}$, was divided into three equal sections. The range $0 \leq z \leq 0.67 \mathrm{~cm}$ in the $\mathrm{z}$-axis demonstrates the evaporator section; adiabatic section occupies the microchannel from $z=0.67 \mathrm{~cm}$ to $z=1.33 \mathrm{~cm}$, while the condenser section is located between $z=1.33 \mathrm{~cm}$ and $z=2 \mathrm{~cm}$. The pressure on the EE' line changes from $65 \mathrm{~Pa}$ to $-45 \mathrm{~Pa}$ and presents a pressure difference of 
$110 \mathrm{~Pa}$ between the evaporator and the condenser. $\mathrm{Wu}$ and Peterson [31] reported a pressure difference of $130 \mathrm{~Pa}$ between the evaporator and condenser.

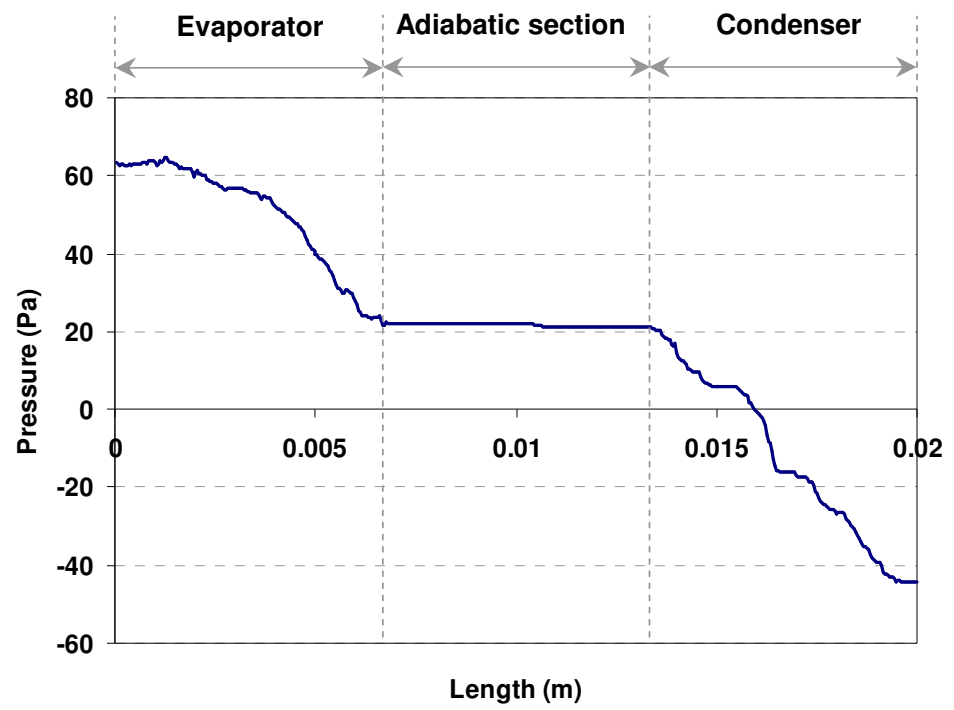

Figure 3-17: Pressure (Pa) along the line EE' for the Run 12

The decrease in the pressure from the evaporator to the condenser determines the direction of vapour flow in the channel. It is evident that the vapour flows from high-pressure region - evaporator - to the low-pressure regions in the condenser section. The steady state pressure difference between evaporator and condenser guarantees the continuous flow of the vapour within the microchannel. One interesting point in Figure 3-17 is the relatively constant pressure along the adiabatic section. There is no source of pressure difference between two ends of the adiabatic section, except the pressure losses within the channel. A pressure difference of $3 \mathrm{~Pa}$ was monitored between the two ends of adiabatic section.

Vapour conservative volume fraction along the line EE' in the microchannel is shown in Figure 3-18. Conservative volume fraction data are not common in the post processing. It is used by the CFX solver and may exceed unity. Furthermore, it may not sum to unity over all fluids during the solution. Therefore, it is only an 
index of the fluid density in order to evaluate the behaviour of the fluid flow in the model. Figure 3-18 demonstrates that the vapour volume fraction results are in accordance with the pressure results. The increase of the vapour pressure within the evaporator (i.e. $0 \leq z \leq 0.67 \mathrm{~cm}$ ) indicates the increase of the molecular energy, and leads to a lower density, or conservative volume fraction. Therefore the results of vapour conservative volume fraction in Figure 3-18 are in accordance with the pressure results. Figure 3-18 can be considered as a representative for the density behaviour along the line EE', in the microchannel.

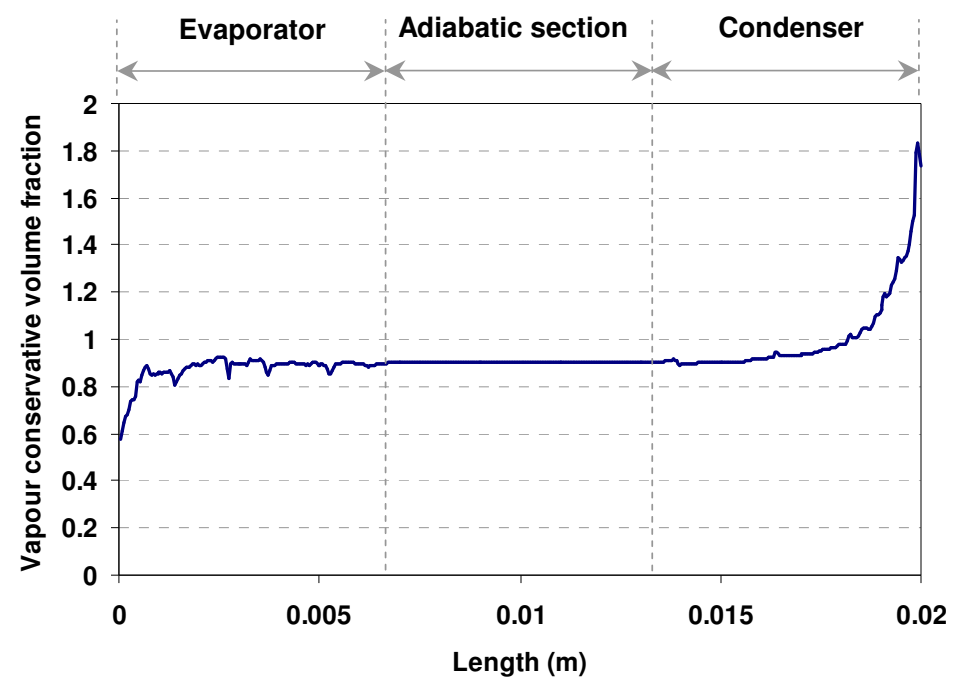

Figure 3-18: Water vapour conservative volume fraction along the line EE' for the Run 12

Further characteristics of the vapour flow can be addressed through the evaluation of velocity field in the microchannel. The velocity field was investigated in the plane of symmetry and therefore the out of plane component of the velocity was not considered. Thus, the two remaining components were the axial or z-direction component and the vertical or y-directional component. Figure 3-19 shows the vertical velocity of the vapour flow along the line EE'. The primary cause of the vertical velocity in the microchannel is the phase change phenomenon. In the evaporator, the vaporized liquid moves from the corners towards the central regions of the channel. Three different flows from three sharp corners lead to a 
complicated flow pattern in the central regions of the channel. By analogy, the vertical velocity in the condenser is complicated and contains numerous fluctuations. However, the most important feature in Figure 3-19 is that the vertical velocity in the adiabatic section is zero. Negligible vertical velocity in the adiabatic section demonstrates the negligible phase change in this section of the microchannel. Therefore, a unidirectional flow for the vapour phase is expected in the adiabatic section. Thus, the liquid, in the sharp corners of the microchannel, should only flow axially within the adiabatic section.

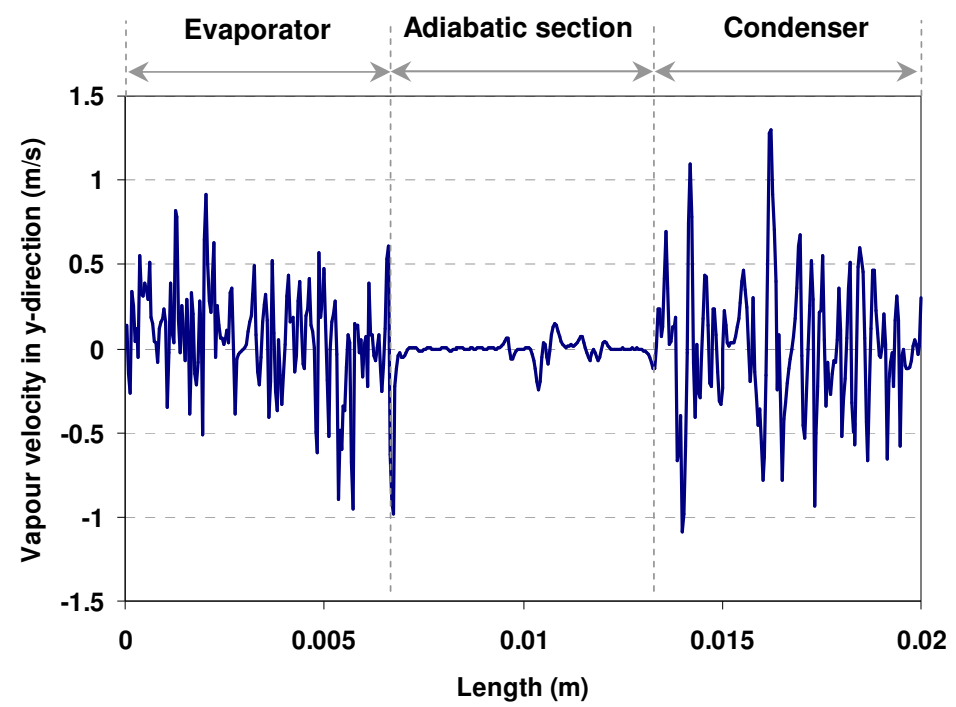

Figure 3-19: Water vapour vertical velocity (m/s) along the line EE' for the Run 12

The vapour axial velocity along the line EE' is shown in Figure 3-20. In the evaporator $(0 \leq z \leq 0.67 \mathrm{~cm})$, the liquid vaporized and the vapour velocity increased from zero to a maximum value of almost $1 \mathrm{~m} / \mathrm{s}$. This velocity stayed constant within the adiabatic section $(0.67 \leq z \leq 1.33 \mathrm{~cm})$. The vapour condensed in the condenser section $(1.33 \leq z \leq 2 \mathrm{~cm})$ and the velocity went back to zero. However, as a result of condensation process in the condenser, the suction of the vapour phase (according to Figure 3-17) caused the axial velocity to reach a maximum value. The velocity of $1 \mathrm{~m} / \mathrm{s}$ in the adiabatic section corresponds to the 
Reynolds number of 0.27. Suman and Hoda [38] investigated a microchannel with effective length of $2 \mathrm{~cm}$ and demonstrated a maximum velocity of $0.7 \mathrm{~m} / \mathrm{s}$ in the beginning of the condenser section.

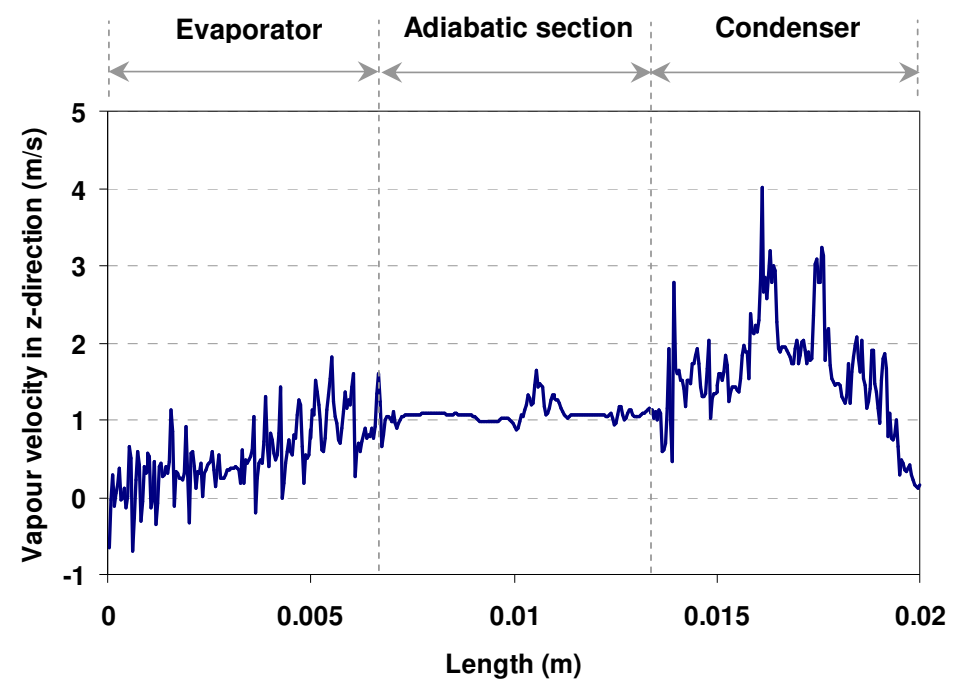

Figure 3-20: Water vapour axial velocity $(\mathrm{m} / \mathrm{s})$ along the line EE' for the Run 11

The constant velocity of the vapour flow during the adiabatic section demonstrated the negligible losses in that zone. The fluctuations in the result of Figure 3-20 were the results of the complex two-phase flow. The entrainment phenomenon in the evaporator and condenser sections, as well as the continuous phase change in these sections, led to highly unstable results. However, the model accurately predicted that the vapour flow accelerates in the evaporator section and keeps the constant velocity in the adiabatic section. A maximum velocity within the condenser section implies the condensation process and the suction of vapour phase. The rest of the loop is completed by the liquid phase going back from the condenser to the evaporator section.

As a result of the steady state situation, the amount of vapour mass flow rate, that crosses a given cross-section, should be equal to the amount of liquid mass flow rate, which is in the opposite direction. That implies the balance of the phase 
changes in evaporator and condenser, and demonstrates that the micro heat pipe is operating under steady state conditions. The mass flow rate depends on the density of the fluid, and the dot product of the flow velocity and the normal vector of the area. Therefore, assuming water density of $997 \mathrm{~kg} / \mathrm{m}^{3}$ and vapour density of $0.231 \mathrm{~kg} / \mathrm{m}^{3}$, vapour velocity in the order of $0.0002 \mathrm{~m} / \mathrm{s}$. Suman and Hoda [38] estimated a range of $0.0001 \mathrm{~m} / \mathrm{s}$ and $0.004 \mathrm{~m} / \mathrm{s}$ for water velocity.

The axial velocity of the liquid phase is shown in Figure 3-21. The line FF' was selected to investigate the behaviour of water in the corners. This line has a distance of $3.2 \mu \mathrm{m}$ from the bottom line $\mathrm{CD}$. The required precision to capture a velocity in the order of $0.0002 \mathrm{~m} / \mathrm{s}$ was not achieved through 200 iterations, and therefore this velocity is not distinguishable in Figure 3-21. However, one important point in this figure is the high velocity of the liquid phase in the beginning of condenser. The high amount of condensation, along with the high velocity of the vapour phase in the beginning of the condenser, pushes the condensed water towards the adiabatic section.

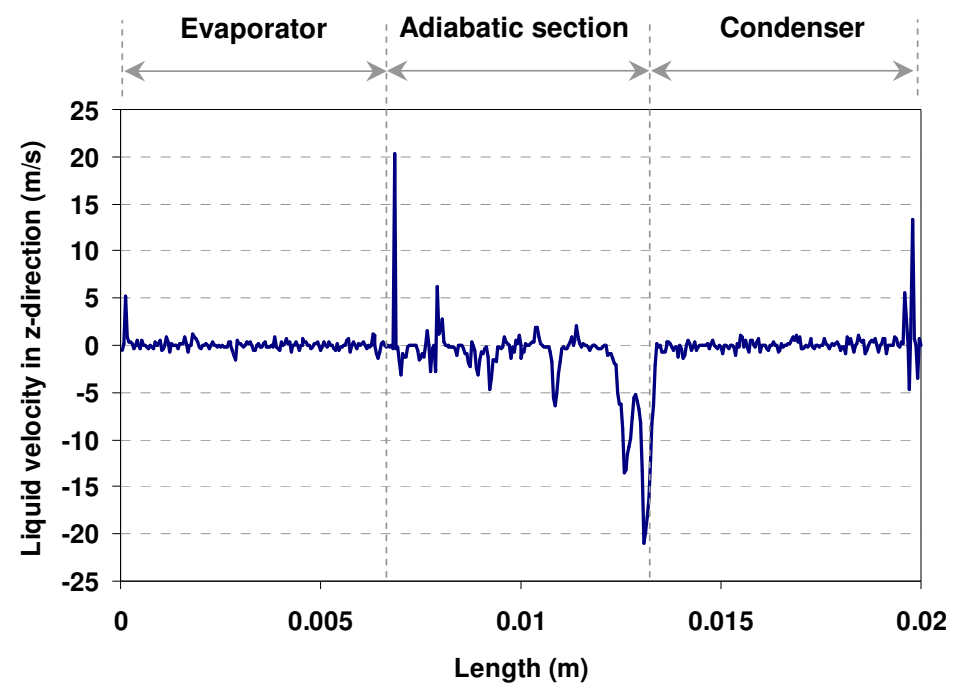

Figure 3-21: Water axial velocity (m/s) along the line FF' for the Run 12 
Besides Figure 3-13 that considered the interphase mass transfer rate in the form of contour, Figure 3-22 presents the same type of results for another case. The complex nature of phase change in the evaporator and condenser led to complicated phase change pattern, however a few interesting points are evident in this figure. Firstly, the amount of phase change in the adiabatic section was negligible. Furthermore, significant amount of phase change took place in the beginning of condenser and evaporator sections. These results are in accordance with the graphical results shown in Figure 3-13.

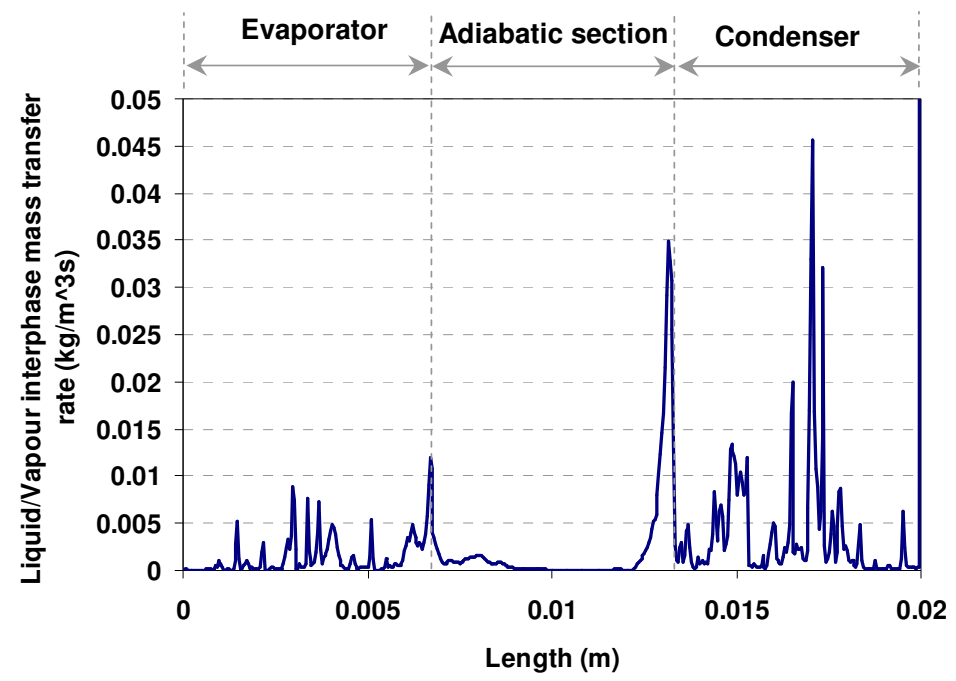

Figure 3-22: Water/water vapour interphase mass transfer rate $\left(\mathrm{kg} / \mathrm{m}^{3} \mathrm{~s}\right)$ along the line EE' for the Run 11

As mentioned in chapter 2, significant investigations have been performed to evaluate the maximum heat transport capacity of thermal microfluidic devices. Figure 3-23 evaluates the behaviour of the model under over loading situations. Figure 3-23-a demonstrates the water vapour conservative volume fraction which is a measure of the vapour density. A dramatic decrease in the water vapour conservative volume fraction is evident at $z=0.0125 \mathrm{~m}$. According to Figure 3-23-a, the effective length of the micro heat pipe is defined as the distance from the condenser end where the water vapour conservative volume fraction goes to zero. It means that under high values of thermal load the micro 
heat pipe can transport heat as far as the effective length. The corresponding results for water vapour axial velocity are shown in Figure 3-23-b. It is evident that the model behaves in an unstable manner after the effective length. That is a result of the low volume fraction of the vapour phase. Since the rest of the microchannel is almost empty, the behaviour of the vapour phase is not reliable.

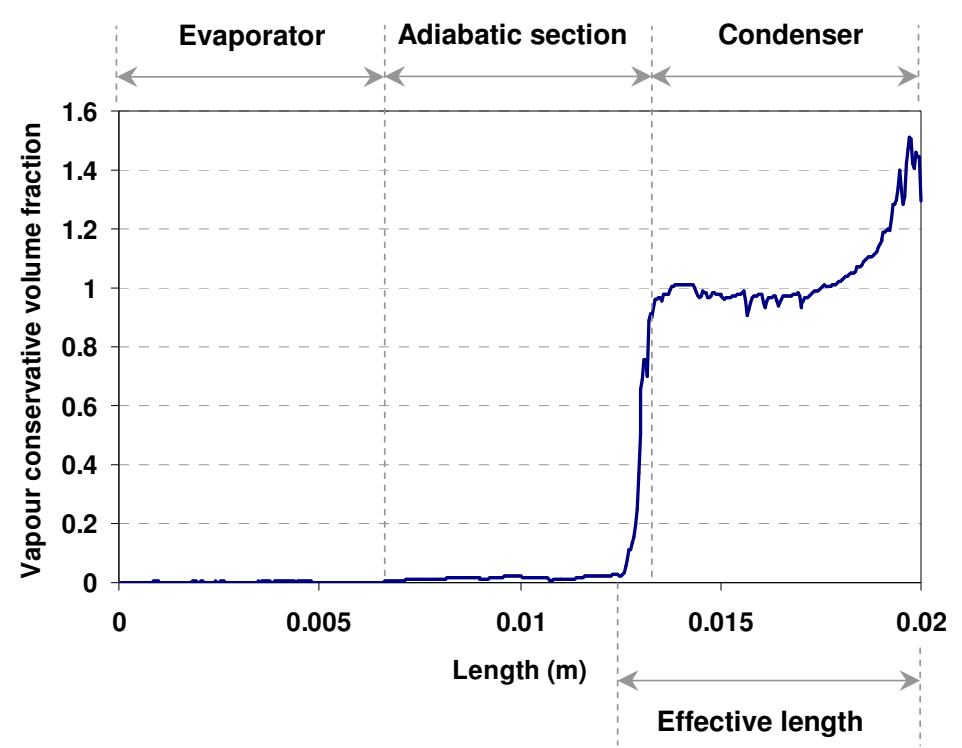

(a)

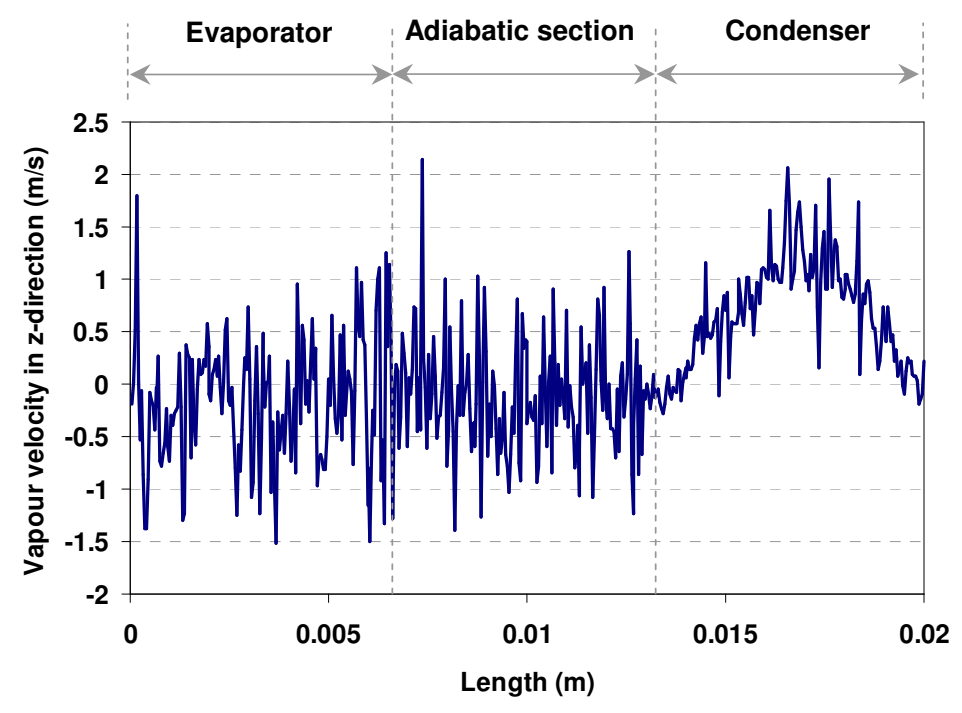

(b)

Figure 3-23: a) Water vapour conservative volume fraction, and b) water vapour axial velocity $(\mathrm{m} / \mathrm{s})$ along the line EE' for the Run 7 
In order to consider the effect of over loading, the effect of filling ratio on the effective length of the micro heat pipe is investigated; see Figure 3-24. First, the effective length was divided by the total length of the channel, to obtain the dimensionless effective length. Therefore, a dimensionless effective length of 0.33 indicates the end of condenser and beginning of adiabatic section. The microchannel filling ratio was considered between $25 \%$ and $75 \%$. Figure $3-24$ shows the dimensionless effective length as a function of the filling ratio. The results show that under over loading conditions, the increase of the filling ratio leads to the increase of the effective length. Thus, under very high thermal loads, micro heat pipes with higher filling ratio can transport heat to a farther distance.

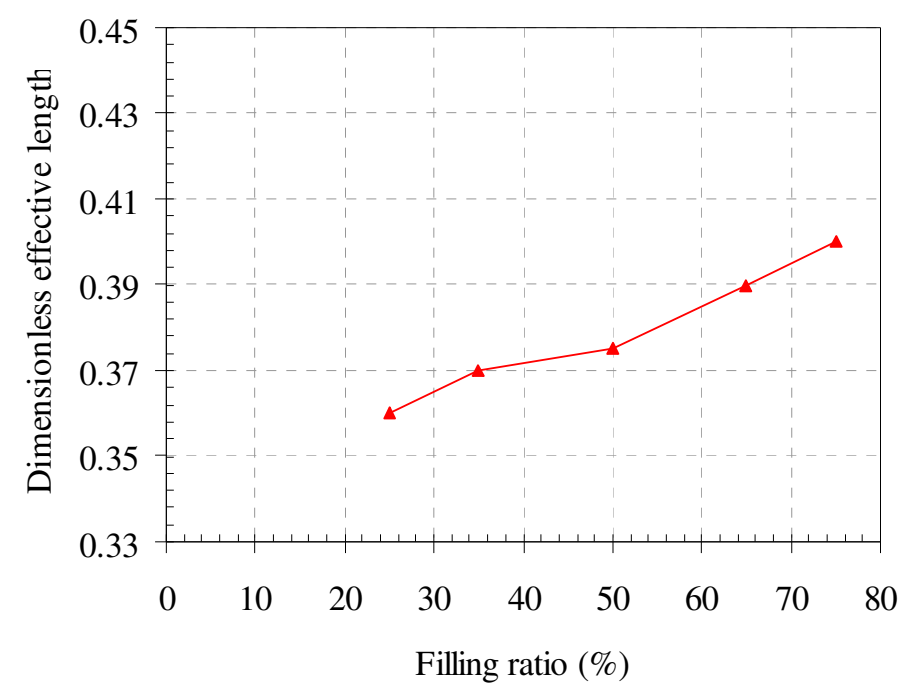

Figure 3-24: Dimensionless effective length as a function of liquid filling ratio

In this section, two-phase flow in a micro heat pipe was simulated. Different boundary conditions and simulation settings were applied to different models and the convergence of these models was investigated. The stability of the results was studied briefly, and it was found that the models with more severe boundary conditions showed more instability. Next, the results of these models were presented and compared with the literature. Good correlation demonstrated the 
validity of the results obtained by finite element software ANSYS CFX-5.7.1. Finally, the results from different analyses were compared to each other and the behaviour of the system with different liquid filling ratio was investigated. 


\section{Chapter 4 Macro modelling}

This chapter presents the analysis of various microchannel configurations to optimize microfluidic chips. As demonstrated in the previous chapter, circular cross-section presented the best performance, however the lack of sharp edges reduces the capillary pressure and limits the applications only to active microfluidic chips. Accordingly, in this chapter, different cross-sections with sharp edges, such as rectangular, triangular, and trapezoidal, are employed. Alongside with the optimization of microchannel configurations, geometric parameters of cross-section are investigated in this chapter. Therefore, geometric parameters of an optimum microfluidic chip can be determined based on the materials presented in this chapter.

Various configurations of microchannels in microfluidic chips are based on the design requirements. In order to optimize the configuration of microchannels in a microfluidic chip, two questions should be dealt with: "how to optimize the crosssectional parameters of a microchannel", and "how the microchannels should be located together to present the optimum thermal performance”. In order to answer these basic questions three steps are followed to optimize the configuration of microchannels. In the first step, a single microchannel embedded in a substrate is modeled. Good understanding of the behaviour of a single microchannel can help to realize the interference of microchannels in an array or three-dimensional configuration. The second step consists of the optimization process for a planar array of microchannels. Based on the results of a single channel modelling, the interference of adjacent microchannels in an array of microchannels is distinguishable. Each application of thermal microfluidic chips requires an optimization process based on the operational parameters of the given application. This matter of fact is considered in the optimization of microchannel array and the results are presented in the form of dimensionless design charts. The dimensionless design charts enable the designer to optimize the configuration of microchannels based on the operational requirements of a given thermal 
microfluidic chip. In the last step, a three-dimensional investigation is performed to survey the interconnected configuration of microchannels.

The macro modelling approach considers the macro phenomena in microfluidic chips. Based on the results of the previous chapters, microchannels are modeled as homogeneous materials with uniform thermophysical properties. Complicated fluid flow phenomena in the microchannels do not have significant effects on the macro behaviour of a thermal microfluidic chip; therefore, by ignoring the micro phenomena inside the channels, a more general domain of geometrical parameters can be considered.

\subsection{Single-phase model}

\subsubsection{Array of microchannels}

Two models, each containing an array of five microchannels with spacing of 1.07 $\mathrm{mm}$ and $1.2 \mathrm{~mm}$, were simulated in ANSYS Workbench 10.0. The models were meshed with around 12500 rectangular solid elements. A temperature boundary condition of $85^{\circ} \mathrm{C}$ applied to the inside surface of grooves, and a convection boundary condition was simulated for the bottom surface. Uniform thermophysical properties were allocated to the channel, and the interaction of microchannel and the surrounding material was considered. Microchannel properties were identical to the thermophysical properties that were selected in the micro modelling of a single microchannel. Based on the selected manufacturing technique - direct-write method - a typical epoxy with relatively high thermal conductivity was selected as the surrounding material. Table 4-1 shows the material properties for epoxy and microchannel.

Figure 4-1 shows the steady state temperature distribution in the two cases. For the microfluidic chip with $1.07 \mathrm{~mm}$ gap distance, the temperature at the top surface was almost uniform. For a larger gap distance, the area between the channels had a lower temperature compared to the microchannels $\left(\Delta T=0.6^{\circ} \mathrm{C}\right)$. 
Table 4-1: Material properties for the epoxy and the microchannels.

\begin{tabular}{lcc}
\hline Property & Epoxy & Microchannel $^{*}$ \\
\hline Density $\left(\mathrm{kg} / \mathrm{m}^{3}\right)$ & 1500 & 225 \\
Specific heat $\left(\mathrm{J} / \mathrm{kg}^{\circ} \mathrm{C}\right)$ & 940 & 2500 \\
Thermal conductivity $\left(\mathrm{W} / \mathrm{m}^{\circ} \mathrm{C}\right)$ & 1 & 400 \\
Thermal expansion $\left({ }^{\circ} \mathrm{C}^{-1}\right)$ & $30 \times 10^{-6}$ & - \\
Young's modulus $(\mathrm{GPa})$ & 4 & - \\
Poisson's ratio & 0.35 & - \\
\hline
\end{tabular}

* Effective properties based on a fluid content of $22.5 \%$ by volume.
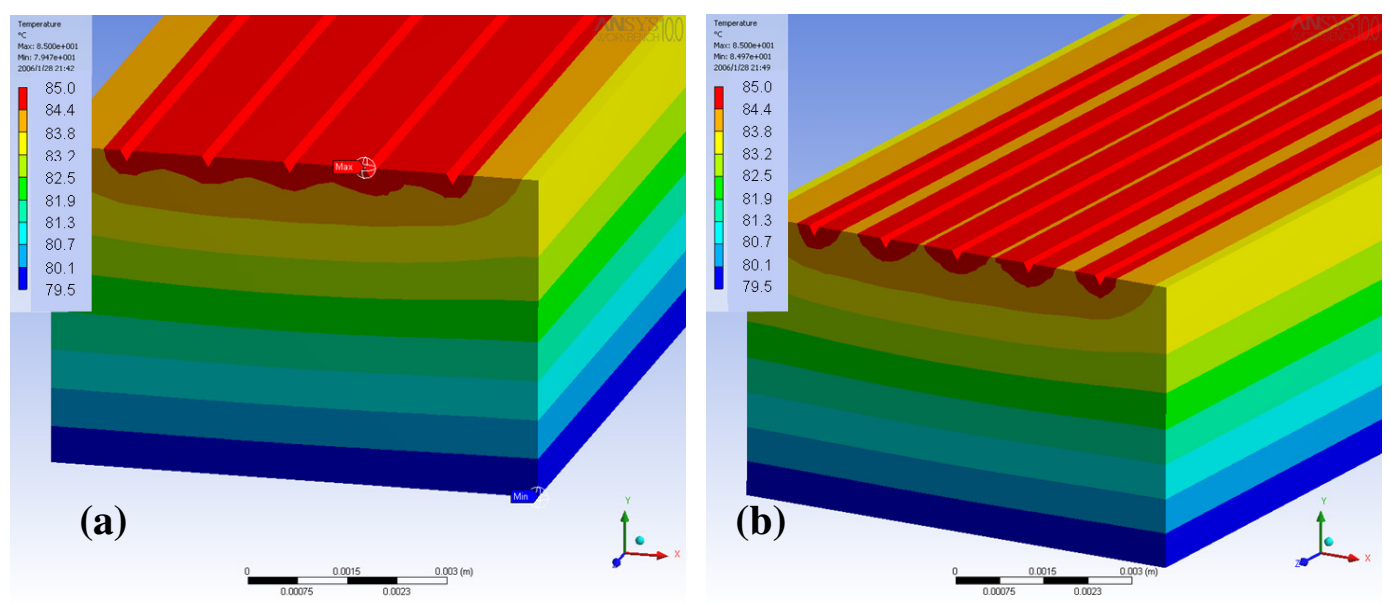

Figure 4-1: Steady state temperature contour $\left({ }^{\circ} \mathrm{C}\right)$ for array of channels with a) $1.07 \mathrm{~mm}$, and $\left.\mathbf{b}\right)$ $1.2 \mathrm{~mm}$ gap distance between the channels

Figure 4-2 shows the total heat flux for the two arrays of microchannels. It is evident that the ranges of heat flux penetration in these cases cause interference between the channels. One other important fact is the difference between the middle and lateral channels. Two channels in both sides surround the middle channels, whereas the lateral channels have the sides of microfluidic chip in one side. Therefore an array of five microchannels seems promising for the geometric optimization of microfluidic chips. 

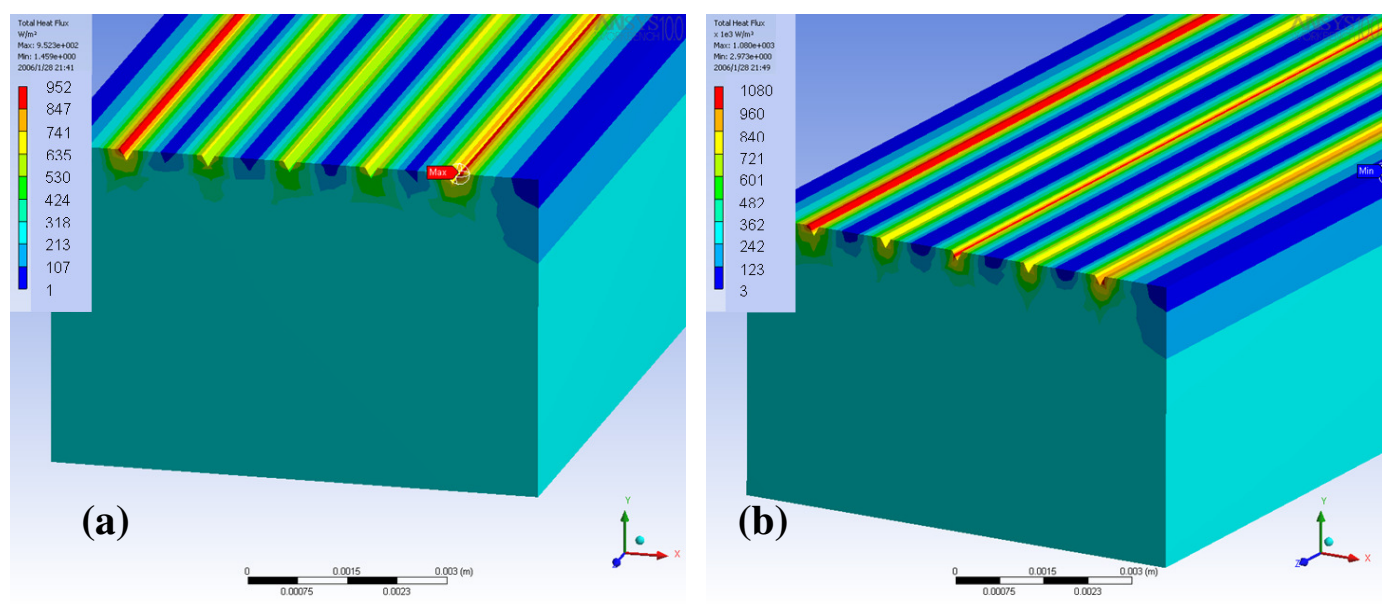

Figure 4-2: Total heat flux $\left(\mathrm{W} / \mathrm{m}^{2}\right)$ for array of channels with a) $1.07 \mathrm{~mm}$, and b) $1.2 \mathrm{~mm}$ gap distance between the channels

In order to optimize the microfluidic chip geometry, the heat transfer process of an array of microchannels was modeled using ANSYS Workbench 10.0 finite element software. The microchannels were modeled as homogeneous materials with equivalent material and standard epoxy properties were used for the microchannels surrounding material. The material properties of microchannels and epoxy are listed in Table 4-1.

A parametric model was developed to investigate the effect of geometry on the thermal behaviour of an array of five microchannels. The parametric model provides the ability to change the geometry and boundary conditions in the adiabatic section and to obtain the optimum configuration. Figure 4-3 shows the geometric parameters used in the parametric model. A parametric trapezoidal cross-section was selected for the model. The parametric trapezoid can represent the triangular, rectangular, and trapezoidal cross-sections. A triangle can be simulated by setting the channel base width $\left(W_{b}\right)$ equal to zero; while setting the channel base width $\left(W_{b}\right)$ equal to the channel top width $\left(W_{t}\right)$ leads to a rectangular cross-section. 


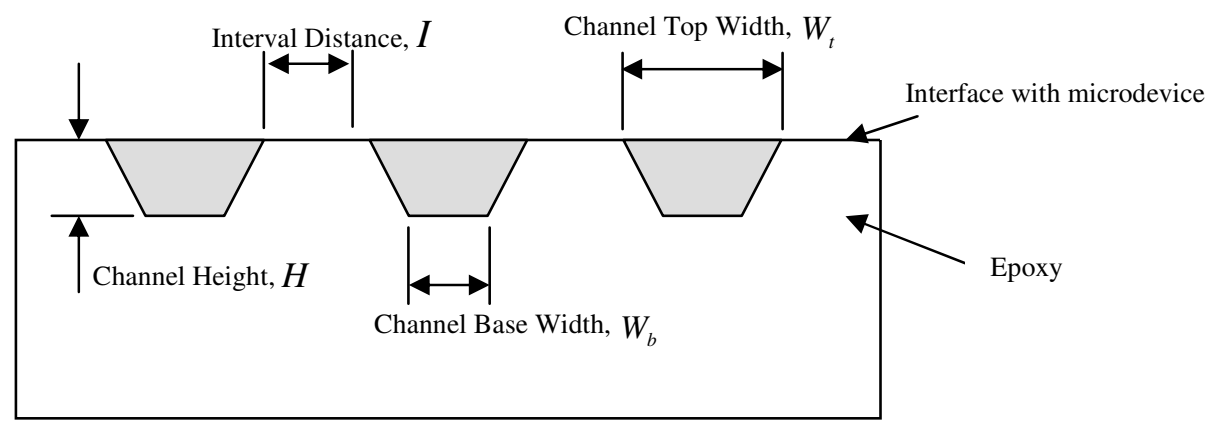

Figure 4-3: Geometric parameters used in the parametric model

The adiabatic section was meshed with 12500 elements and the model boundary conditions were applied according to Figure 4-4. The hot source $\left(T_{h}\right)$ and cold sink $\left(T_{c}\right)$ temperatures were $90^{\circ} \mathrm{C}$ and $10^{\circ} \mathrm{C}$, respectively; while the ambient temperature $\left(T_{a}\right)$ was set to $40^{\circ} \mathrm{C}$. The heat fluxes at the hot source $\left(\dot{Q}_{i}\right)$ and at the cold $\operatorname{sink}\left(\dot{Q}_{o}\right)$ were $15500 \mathrm{~W} / \mathrm{m}^{2}$ and $-15500 \mathrm{~W} / \mathrm{m}^{2}$, respectively. The convection heat transfer coefficient $(h)$ was set to $200 \mathrm{~W} / \mathrm{m}^{2} \mathrm{~K}$. As shown in the preliminary investigation of micro modelling (section 3.1), in the analysis of the adiabatic section, the heat flux can be assumed to be in the microchannel axial direction.

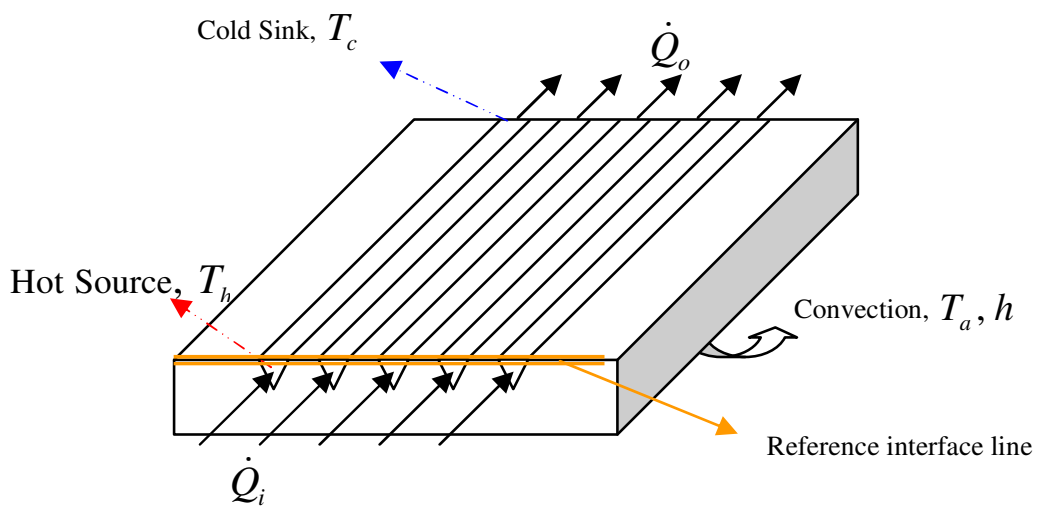

Figure 4-4: Typical boundary conditions applied to the adiabatic section of microchannels array

\subsubsection{Steady state model}

The efficiency of the microchannels array was investigated by the steady state temperature distribution at the interface between the microchannels and the 
microelectronic device (Figure 4-3). Since controlling the temperature and the heat flux in the microdevices are the main objectives of thermal microfluidic chip, the effect of different geometric parameters on the interface temperature distribution is of particular interest. Given that the microchannels were modeled as high conductive materials, in the hot source area, the microchannel temperature was higher than the temperature in the surrounding epoxy and the ambient. Similarly, in the cold sink region, the microchannel temperature was lower than that of surrounding epoxy, and that caused important temperature gradients. In the following analysis, the beginning of the adiabatic section, shown by the reference interface line in Figure 4-4, is analyzed.

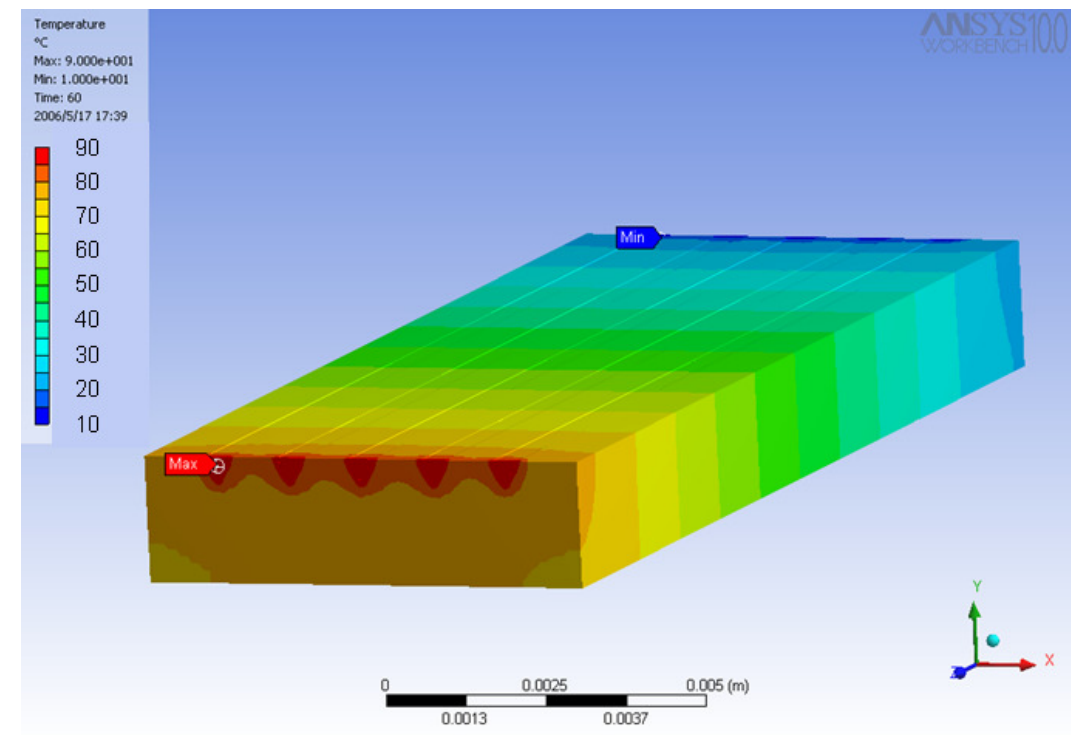

Figure 4-5: Steady state temperature contour $\left({ }^{\circ} \mathrm{C}\right)$ for an array of channels. Each color shows a temperature range of $5^{\circ} \mathrm{C}$.

Figure 4-5 shows the temperature contour for a planar microfluidic chip. It is evident that the geometric parameters, such as channel height, have significant effects on the temperature distribution over the entire microfluidic chip. It was found that the temperature distribution in every cross-section follows the same pattern. The end cross-section in the adiabatic-evaporator interface is shown in Figure 4-5. The top surface in Figure 4-5 presents the interface between the thermal microfluidic chip and the microelectronic component. Since controlling 
the temperature of the microelectronic component is one of the main objectives of electronics cooling, the temperature at the top surface is of special interests. Given that the pattern of the isotherms in all cross-sections is similar, the interface between the adiabatic and evaporator sections is selected as the representative cross-section. In order to monitor an index of the microelectronic temperature, a line in the representative cross-section is specified as "reference interface line" and investigated. The location of the reference interface line is shown in Figure 4-4.

In the thermal model, the temperature along the reference interface line can be reported in terms of the hot temperature, and be classified according to three ranges: $0.83 T_{h}<T<0.89 T_{h}, 0.89 T_{h}<T<0.94 T_{h}$ and $0.94 T_{h}<T<T_{h}$. Figure 4-6 presents the variation of the steady state interface temperature distribution with the dimensionless microchannel interval distance $\left(I / W_{t}\right.$, see Figure 4-3). The simulations were conducted for a triangular microchannel cross-section with $H=$ $400 \mu \mathrm{m}, W_{t}=400 \mu \mathrm{m}$ and $W_{b}=0$. The percentage reported on Figure 4-6 represents the length of each of these temperature ranges over the total length of reference interface line (Figure 4-4) in a given cross-section. A dimensionless average temperature $\left(T_{a v g}\right)$, which was normalized by dividing by the hot source temperature $\left(T_{h}\right)$ at the interface, was also presented. The results showed that for short microchannel spacing, $I / W_{t}<1$, the interface temperature was very close to the hot source temperature. For $I / W_{t}=1-2$, the interface temperature distribution became less uniform. For $I / W_{t}>2$, significant non-uniformity was observed with an increase in the percentage of interface temperature between $0.83 T_{h}<T<$ $0.89 T_{h}$. Since the amount of heat transfer in a given cross-section is dependent on the number of channels located in that area, for heat removal applications the maximum possible channels that meet the requirements of interface temperature should be chosen. According to the result of Figure 4-6, for heat removal applications, a value of $I / W_{t}=2$ is recommended to have a compromise between the amount of heat transfer and the interface temperature. However, different 
applications, based on the interface temperature, the $I / W_{t}$ ratio can be selected from Figure 4-6 to meet the requirements of a given application.

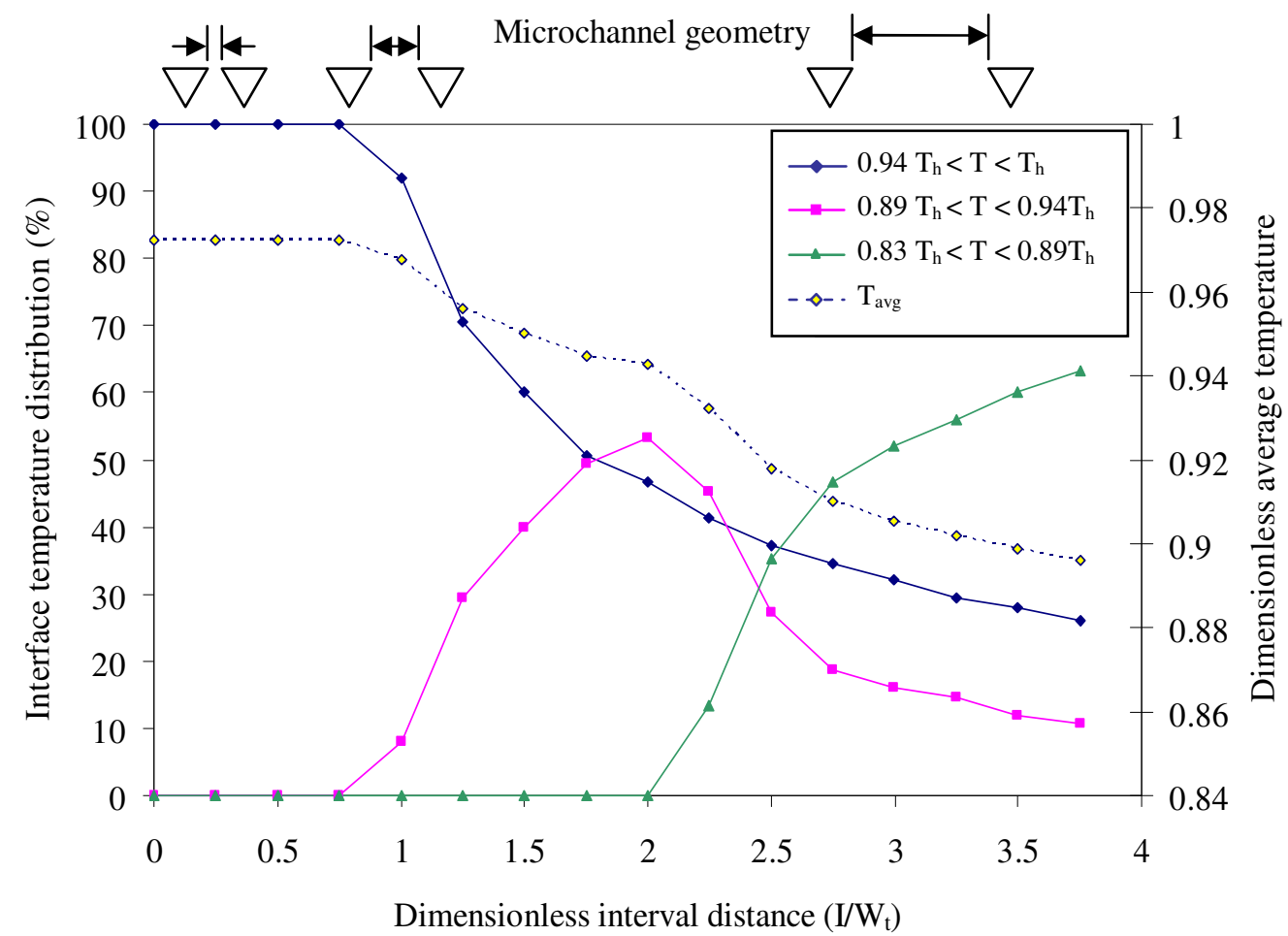

Figure 4-6: Effect of dimensionless interval distance $\left(I / W_{t}\right)$ on the interface temperature distribution, $H=400 \mu m, W_{t}=400 \mu m$ and $W_{b}=0$

Figure 4-7 presents the variation of the steady state interface temperature distribution with the dimensionless microchannel width $\left(W_{b} / W_{t}\right.$, see Figure 4-3). The effect of choosing a triangular cross-section $\left(W_{b} / W_{t}=0\right)$ was considered in Figure 4-6, and in Figure 4-7 a trapezoidal cross-section $\left(W_{b} / W_{t}>0\right)$ was investigated. The simulations were conducted with the following constant parameters: $H=200 \mu m, W_{t}=400 \mu m$ and $I=1500 \mu m\left(I / W_{t}=3.5\right)$. The surface temperatures were classified according to the same procedure described previously and the average temperature of the interface was determined. The results showed that for a narrow microchannel base width, $W_{b} / W_{t}<0.5$, the interface temperature was not very uniform. This result was consistent with the previous analysis for a triangular cross-section with $I / W_{t}=3.5$ as the trapezoidal 
cross-section behaves similarly to a triangular cross-section at small values of $W_{b} / W_{t}$. For $W_{b} / W_{t}>0.875$ the interface temperature distribution became more uniform and at $W_{b} / W_{t}=2.125$ the maximum interface temperature was achieved. As it can be seen in Figure 4-7, for $W_{b} / W_{t}>2.125$ the interface temperature was entirely in the range of $0.94 T_{h}<T<T_{h}$ and increasing $W_{b} / W_{t}$ did not have any effect. According to Figure 4-7, the slope for the $0.94 T_{h}<T<T_{h}$ range was more or less constant up to $W_{b} / W_{t}=1.5$. For $W_{b} / W_{t}>1.5$, a rapid change of the interface temperature was noticeable. This result was confirmed by the variation of the average interface temperature. As a result, Figure 4-7 can be used as a design chart to select the optimized configuration of microchannels for different applications. Particularly, for heat removal applications, a value of $W_{b} / W_{t}=1.5$ is recommended for a maximum heat transfer coefficient and a dimensionless average temperature of 0.944 in the interface.

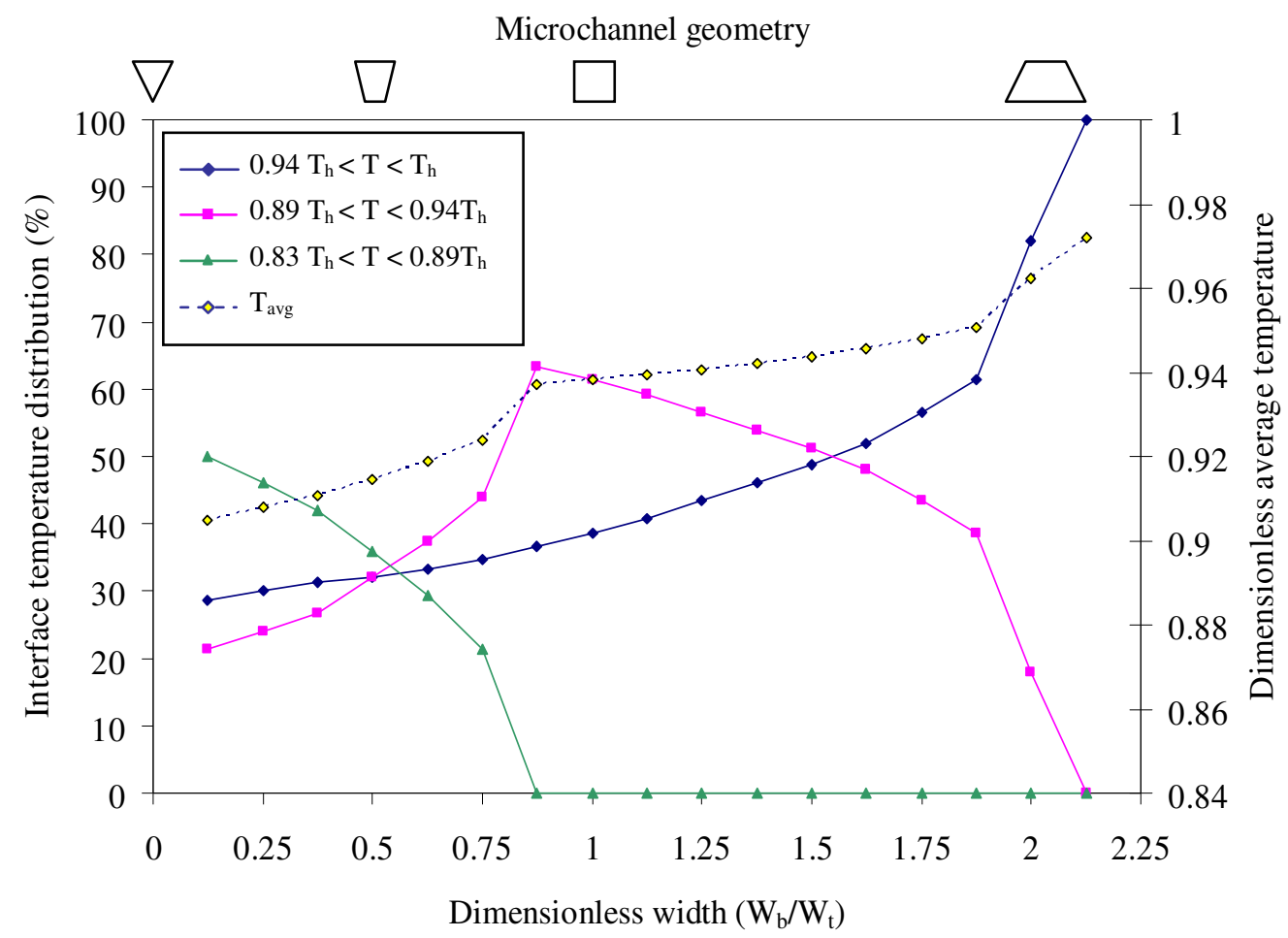

Figure 4-7: Effect of dimensionless width $\left(W_{b} / W_{t}\right)$ on the interface temperature distribution, $H=$ $200 \mu \mathrm{m}, W_{t}=400 \mu \mathrm{m}$ and $I=1500 \mu \mathrm{m}$ 
Figure 4-8 presents the variation of the steady state interface temperature distribution with the dimensionless microchannel height $\left(H / W_{t}\right.$ see Figure 4-3). The simulations were conducted for a triangular microchannel cross-section with $I$ $=800 \mu \mathrm{m}, W_{t}=400 \mu \mathrm{m}$ and $W_{b}=0 \mu \mathrm{m}$. For a very small dimensionless height $\left(H / W_{t}<0.25\right)$ the triangular microchannel geometry was very close to a line. In this case the hot spots $\left(T \approx T_{h}\right)$ on the interface temperature were just located at the channels top surfaces - almost $30 \%$ of the total interface. Considering the $0.94 T_{h}<T<T_{h}$ data, the curvature slope decreased as $H / W_{t}$ approaches 0.875 and increased for $H / W_{t}>0.875$. This behaviour indicated that the heating rate at the interface was high, thus small increases in the $H / W_{t}$ ratio lead to a rapid increase of the interface temperature. The average temperature interface also showed the same trend as observed on Figure 4-8. Similar to Figure 4-6 and Figure 4-7, Figure 4-8 can be used as a design chart in different applications of microfluidic chips. For thermal removal applications, $H / W_{t}=0.875$ satisfied both a minimum interface temperature and a maximum heat transfer. It has to be mentioned that $H / W_{t}=0.875$ corresponds to an equilateral triangle, therefore for triangular microchannels serving in the heat removal application, the best performance was achieved with an equilateral triangle.

The presented design charts (Figure 4-6, Figure 4-7, and Figure 4-8) can be employed to design microfluidic chips operating in different applications. As an example, the design of a microfluidic chip for Polymerase Chain Reaction (PCR) for DNA amplification is presented. The PCR amplification process contains three sectors with different process temperatures: denaturation, annealing, and extension. The annealing sector, which contains the attachment of separated single strand DNA to a specific primer, takes place at $54^{\circ} \mathrm{C}$ [65]. Subsequently, the design process for appropriate microfluidic chip in this application is presented. In this application, the temperature uniformity at the interface has a higher design priority compared to the amount of heat transferred in the microfluidic chip. Figure 4-8 shows that the dimensionless average temperature is very sensitive to microchannel height, especially for $H / W_{t}<0.875$. Therefore, to 
consider the possible difficulties in the manufacturing of microfluidic chip, use of trapezoidal channel is recommended. Figure 4-7 shows less sensitivity of dimensionless average temperature to the dimensionless width for $W_{b} / W_{t}>0.875$. Thus, $W_{b} / W_{t}=1.5$ is suggested to have a dimensionless average temperature between 0.94 and 0.95 . In this case, the microchannel specifications are: $I=1500$ $\mu \mathrm{m}, W_{t}=400 \mu \mathrm{m}, H=200 \mu \mathrm{m}$, and $W_{b}=600 \mu \mathrm{m}$. Applying a $T_{h}=57^{\circ} \mathrm{C}$ causes an average temperature of $54^{\circ} \mathrm{C}$ with a maximum tolerance of $6 \%$ in the entire interface.

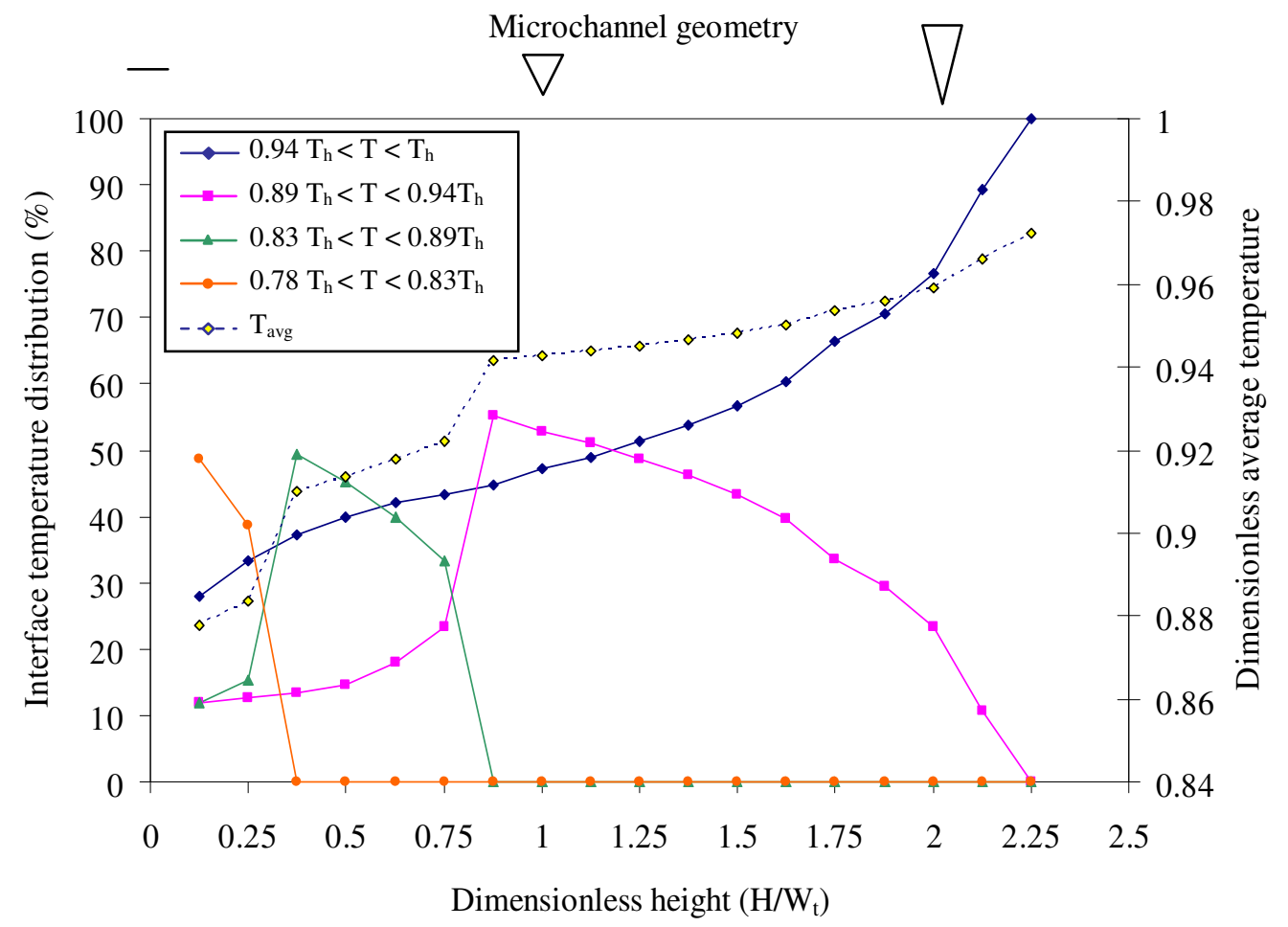

Figure 4-8: Effect of dimensionless height $\left(H / W_{t}\right)$ on the interface temperature distribution, $I=$ $800 \mu \mathrm{m}, W_{t}=400 \mu \mathrm{m}$ and $W_{b}=0 \mu m$

\subsubsection{Transient model}

Thermal microfluidic chips applications involve unsteady conditions, as time dependent changes in the boundary conditions. For instance, in the electronic cooling applications, a change in the power consumption of the electronic 
component leads to changes in heat flux and temperature boundary conditions of the microfluidic chip. Thus, for the transient analysis, the heat fluxes and hot source temperature were ramped to their steady state values - according to steady state modelling - in 5 seconds. Constant conditions were maintained for 20 seconds before they were ramped down to the initial conditions in 5 seconds. With an initial temperature of $22^{\circ} \mathrm{C}$ for the microfluidic chip and $10^{\circ} \mathrm{C}$ for the heat sink, fast ramp rates for the heat fluxes and the hot source temperature were simulated.

The results of the transient heat transfer analysis of the top surface temperature are presented in Figure 4-9 and Figure 4-10. The results presented in Figure 4-9 suggests that the microfluidic chip had a very fast response time, as steady state conditions were almost instantaneously achieved in both the microchannel and the surrounding epoxy. In the worst conditions, a maximum response time of much less than 2 seconds was necessary to reach the steady state conditions. It should be mentioned that in this investigation a homogeneous material was allocated to the microchannels. Therefore, for the real conditions, where the fluid flow determines the thermal performance of the microfluidic chip, a greater time is expected before the steady state conditions.

The evolution of the temperature gradient along the microfluidic chip length with time is presented in Figure 4-10. According to the boundary conditions, the cold sink temperature was kept constant, while the hot source temperature was increased through the time. Aside from the regions close to the heat source and the cold sink, the temperature profile was linear. As observed in the steady state analysis, there was a small temperature difference between microchannels and the surrounding epoxy. The fast convergence of the transient response to the steady state condition indicated that the results of Figure 4-10 correspond to the steady state responses under different boundary conditions of hot source temperature. For example, a transient response after 3.6 seconds in the microchannel can be interpreted as a steady state response with a hot source temperature equal to 74 
percent of the original hot temperature. As a result of Figure 4-10, a single transient response may represent various steady state situations with different boundary conditions.

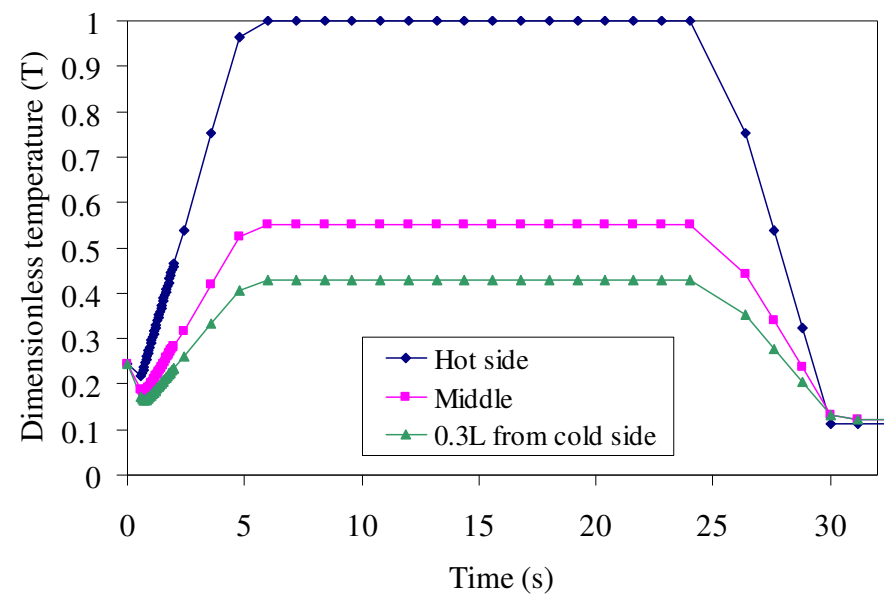

(a)

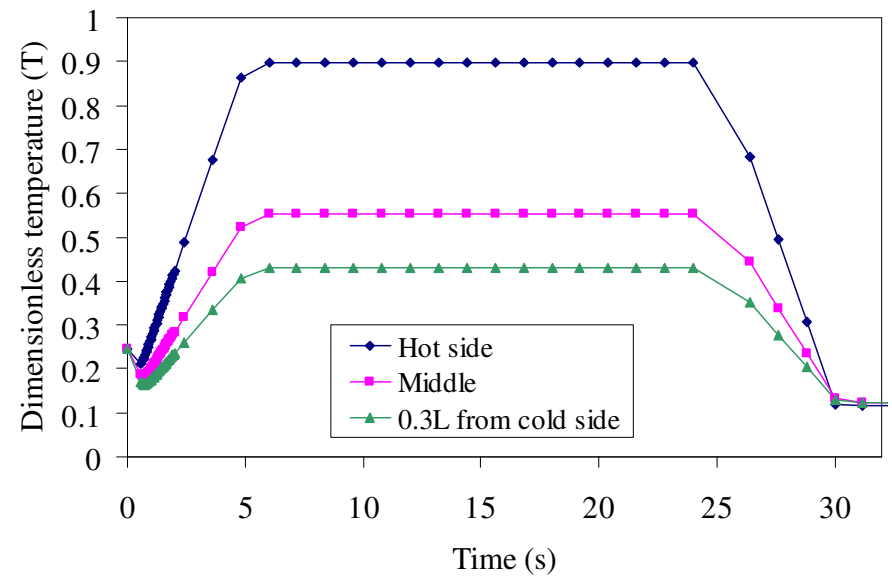

(b)

Figure 4-9: Transient response of microfluidic chip in different positions of top surface for a) microfluidic chip microchannel and b) surrounding epoxy 


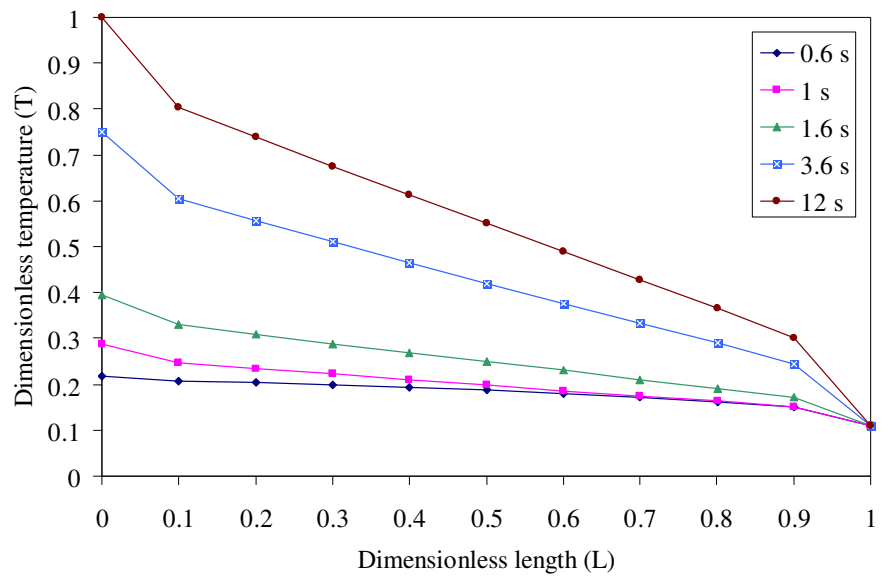

(a)

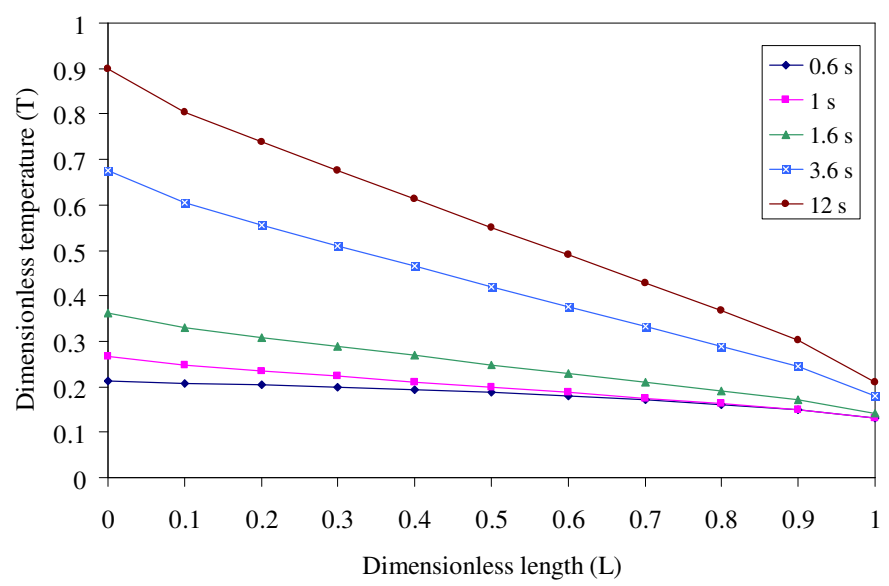

(b)

Figure 4-10: Interface temperature gradient along the microfluidic chip length at different time for a) microfluidic chip microchannel and b) surrounding epoxy

\subsubsection{Three-dimensional configuration of microchannels}

The proposed technique of direct-write assembly [56] allows the manufacture of three-dimensional interconnected microchannels networks. In this technique a three-dimensional scaffold can be deposited on a substrate, and later on be infiltrated with resin in order to provide a complex network of microchannels (Figure 4-11-a). This technique is also able to provide layer-by-layer deposition; see Figure 4-11-b. In this case, the first layer of microchannels is deposited on the 
substrate. Afterwards, the system is infiltrated with resin and solidified to be ready for the deposition of the next layer of microchannels. The sequence of writing and infiltrating can be continued to obtain the final three-dimensional microfluidic chip.

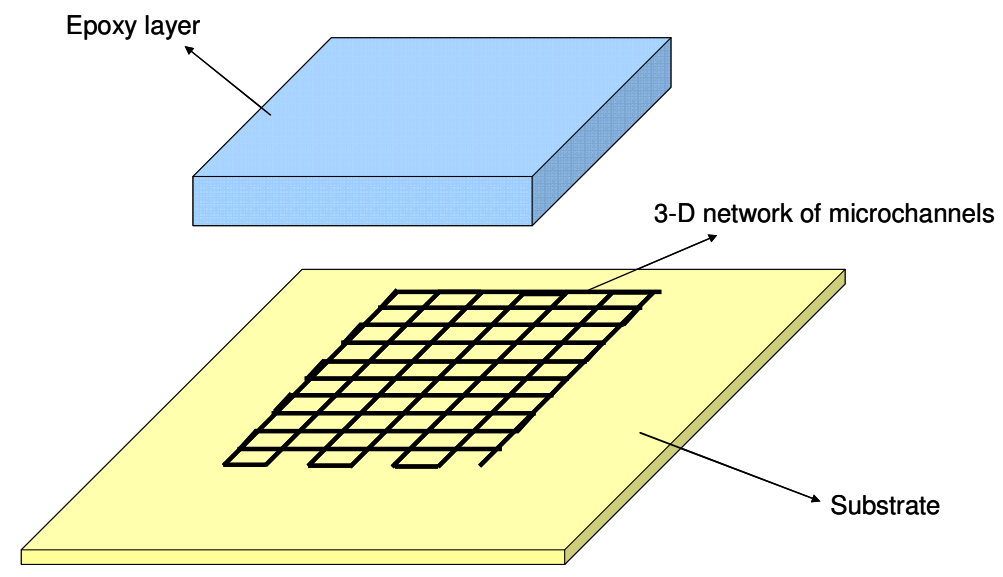

(a)

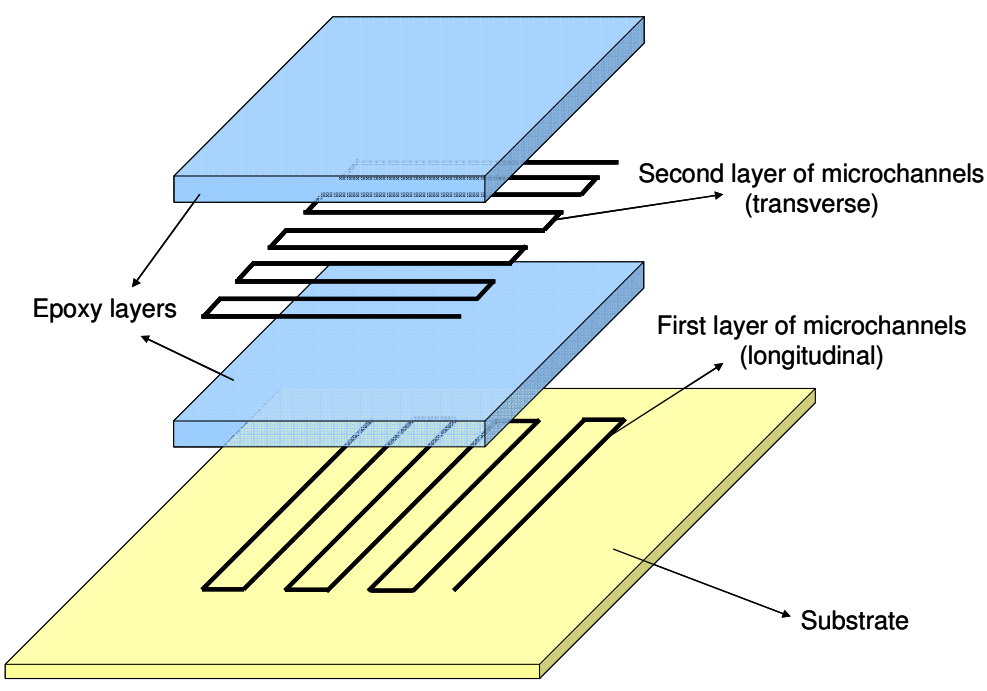

(b)

Figure 4-11: The order of microchannel layers for a) one step infiltration (case1), and b) layer-bylayer infiltration (case 2)

In order to compare the thermal performance of interconnected network technique with the layer-by-layer technique, three different models were examined. The first model (case 1) includes an interconnected network of microchannels with the first 
row of channels in the longitudinal direction of the microfluidic chip (Figure 4-11-a and Figure 4-12-a). In this model, all the different rows of microchannels are connected to each other. The second and the third models are employing the layer-by-layer concept to manufacture the microfluidic chip. As mentioned before, the microfluidic chip can be assumed to be a long plate. Hence, there are two directions in a planar layer: longitudinal and transverse. The second model (case 2) employs longitudinal microchannel for the first layer (Figure 4-12-b), while the third model (case 3) has transverse microchannels in the first layer (Figure 4-12-c). The first layer is in direct contact with the electronic components and extracts the heat from the hot spot. Additionally, within the adiabatic section, it is still attached to the electronic board and a heat flow may occur as a result of temperature difference between the microchannels and the electronic board. Thus, the design of the first layer affects the thermal performance of the microfluidic chip.

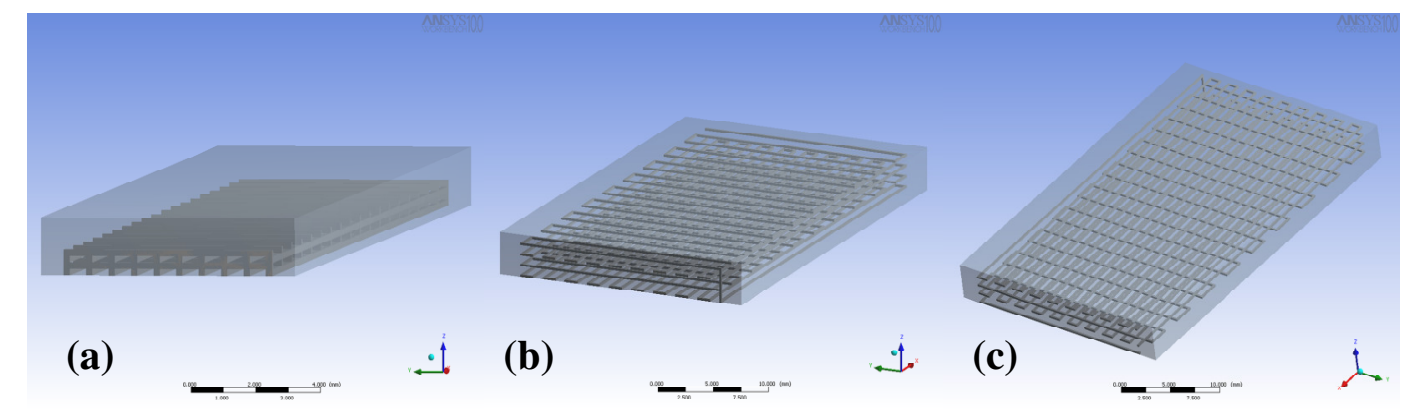

Figure 4-12: Three cases for three-dimensional geometric study, a) case 1, b) case 2, and c) case 3

Thermophysical properties for the microchannels and the surrounding epoxy were defined according to Table 4-1. According to Figure 4-13, in the real circumstances, there are proper interconnections between the crossing microchannels in different layers - due to the weight of the fugitive ink and the injection pressure. Therefore, even in case of circular cross-section, the top layer of fugitive ink presses the bottom layer and the intersection is more than a single point. However, in the modelling approach, two perpendicularly crossing cylinders meet each other only in a single point, which does not allow a proper 
connection of the microchannels. Therefore, in the models, square cross-sections with the sides of $200 \mu \mathrm{m}$ were selected to have appropriate connections.

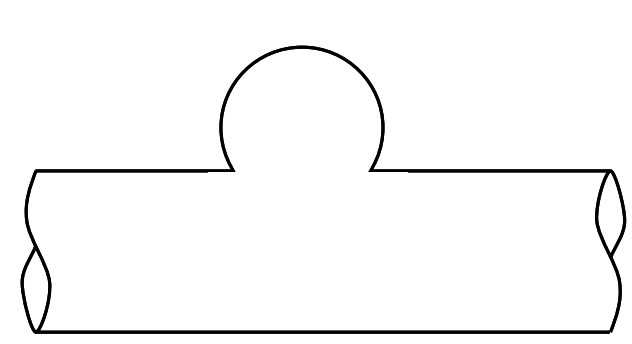

(a)

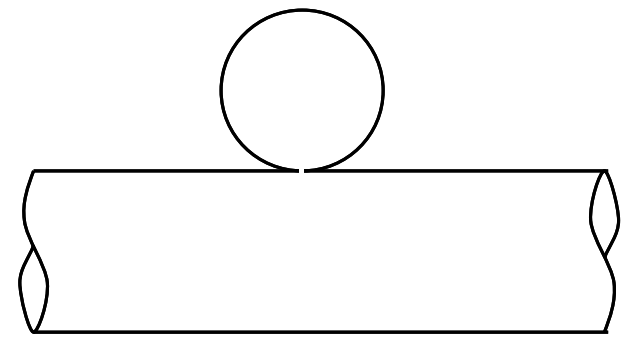

(b)

Figure 4-13: Interconnection between the microchannels in different layers for a) the real, and b) the ideal situation

Figure 4-14 shows the boundary conditions applied to the models. The heat flux $\left(\dot{Q}_{i}\right)$ and temperature $\left(T_{h}\right)$ at the evaporator side of the model were selected to be $50000 \mathrm{~W} / \mathrm{m}^{2}$ and $90^{\circ} \mathrm{C}$, respectively; whereas in the condenser side a heat flux $\left(\dot{Q}_{o}\right)$ of $-50000 \mathrm{~W} / \mathrm{m}^{2}$ and a temperature $\left(T_{c}\right)$ of $10^{\circ} \mathrm{C}$ were applied as boundary conditions. The microelectronic chip temperature $\left(T_{b}\right)$ was set to $60^{\circ} \mathrm{C}$. Convection boundary condition with an ambient temperature $\left(T_{a}\right)$ of $40^{\circ} \mathrm{C}$ was applied to the top surface. The convection heat transfer coefficient $(h)$ was set to $100 \mathrm{~W} / \mathrm{m}^{2} \mathrm{~K}$.

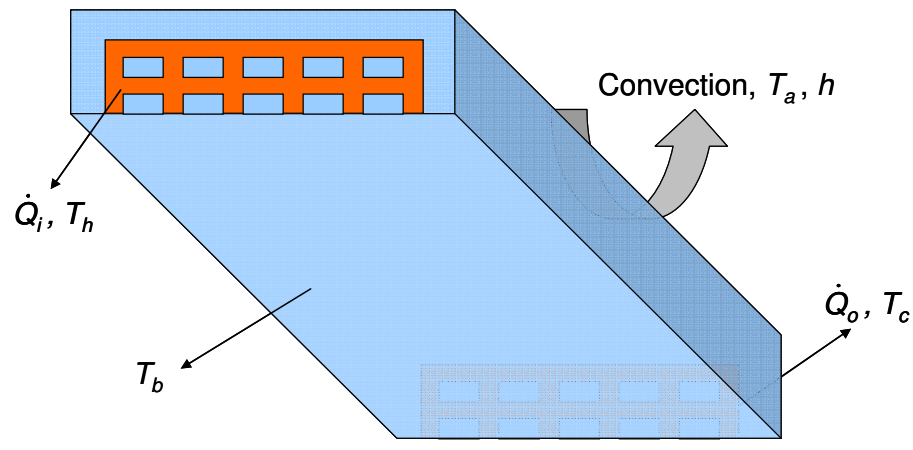

Figure 4-14: Boundary conditions for all three cases in three-dimensional geometric study

Temperature contours of the first model (case 1) are presented in Figure 4-15. In Figure 4-15-a the elements with temperatures higher than $59.9^{\circ} \mathrm{C}$ are shown, 
while Figure 4-15-b illustrates the elements with temperatures higher than $60^{\circ} \mathrm{C}$. Comparing Figure 4-15-a and $b$ results in two important factors. Firstly, the effect of microelectronic chip temperature, and secondly, the low temperature gradient along the microchannels. Since the first layer of microchannels is directly connected to the chip, the microelectronic temperature has a significant effect on the thermal performance of the entire microfluidic chip. Due to the temperature difference, the heat is transfers through the microelectronic chip even in the microfluidic chip adiabatic section. This indicates the importance of the first layer of microchannels. Moreover, the high thermal conductivity of the microchannels causes a small temperature gradient along the channels.
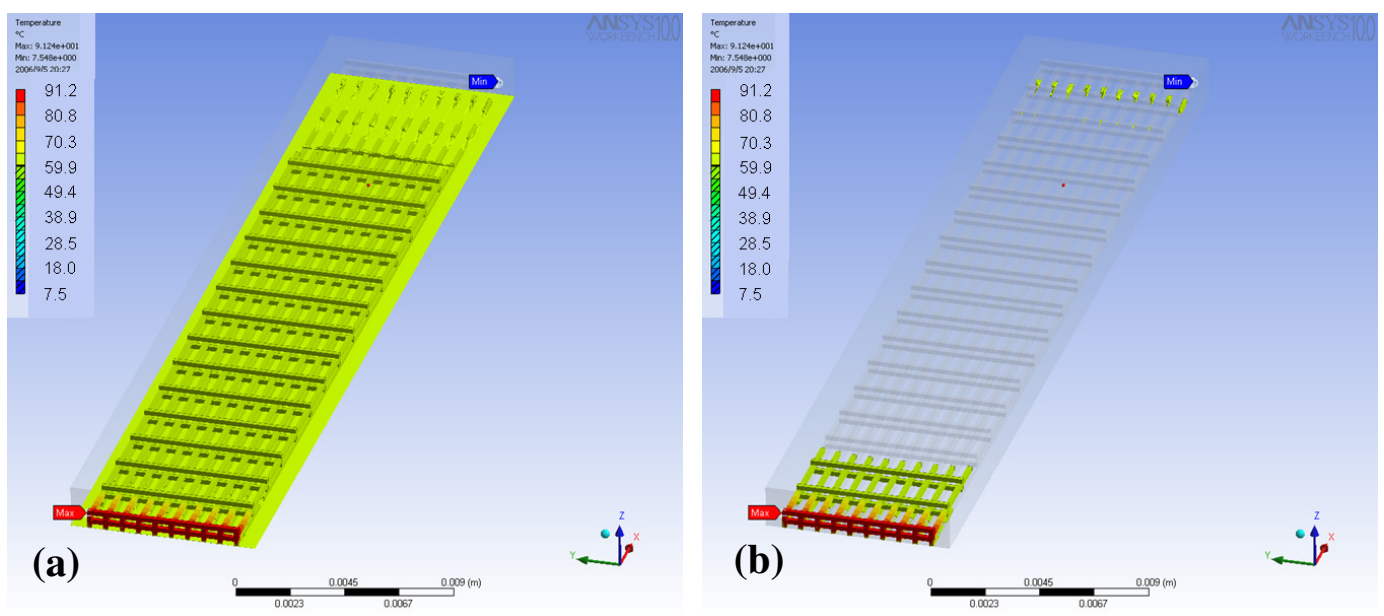

Figure 4-15: Temperature contours in ${ }^{\circ} \mathrm{C}$ (case 1) for a) $\mathrm{T}>59.9^{\circ} \mathrm{C}$, and b) $\mathrm{T}>60^{\circ} \mathrm{C}$

The results of the investigation on the thermal flux of the interconnected model are illustrated in Figure 4-16. Total heat flux from the front view (Figure 4-16-a), side view (Figure 4-16-b), and top view (Figure 4-16-c) show the thermal behaviour of the microfluidic chip. The amount of heat transfer inside the surrounding epoxy was negligible, compared to the microchannels. Additionally, according to Figure 4-16-a, the maximum heat flux occurred in the interface of the microfluidic and the microelectronic chips. That was a result of temperature gradient between the microchannel and the microelectronic chip. Along the axial direction of microchannels, this temperature gradient decreased, such that in some 
points in the middle of the microfluidic chip, the microchannels temperature was equal to that of the electronic chip. The top layers of microchannels were less affected by that temperature gradient and had higher heat fluxes through the microchannels length (Figure 4-16-b). Finally, Figure 4-16-c illustrates the small difference between the lateral and central channels.

As mentioned before, in order to obtain the best thermal performance in the microfluidic chips, three different models were simulated. Figure 4-17 shows all the three models for two cases: the top row pictures show the elements with temperatures higher than $59.9^{\circ} \mathrm{C}$, and the bottom row pictures illustrate the three models including the elements with temperatures higher than $60^{\circ} \mathrm{C}$.

Figure 4-17 demonstrates the effect of connections between different layers of microchannels. For case 1 (Figure 4-17-a and d), the interconnected channels led to a higher heat transfer between electronic and fluidic chips, and that could constitute a good design even for the evaporator and the condenser sections. In this case, the heat was extracted from the hot source, conveyed to the higher layers of microchannels and transferred to the cold side of the microfluidic chip. Since the microchannels top layers possessed higher heat fluxes, smaller interactions with the electronic chip in the adiabatic section occurred in the first case - compare to the other cases. However, case 2 and 3 did not provide connections between the microchannel layers, and therefore, the unwanted heat transfer in the adiabatic section was higher in these cases. Furthermore, According to Figure 4-17, case 1 presented lower temperature gradient compared to case 2 and 3. A comparison between Figure 4-17-a and d demonstrates that there was a significant number of elements with temperatures between $59.9^{\circ} \mathrm{C}$ and $60^{\circ} \mathrm{C}$. However, for case 2 and 3, the number of elements with temperatures between $59.9^{\circ} \mathrm{C}$ and $60^{\circ} \mathrm{C}$ are less than case 1 . Thus, case 1 demonstrated a better thermal performance in the longitudinal direction. In the next investigation fluid flow and heat transfer is examined for the interconnected network of microchannels. 

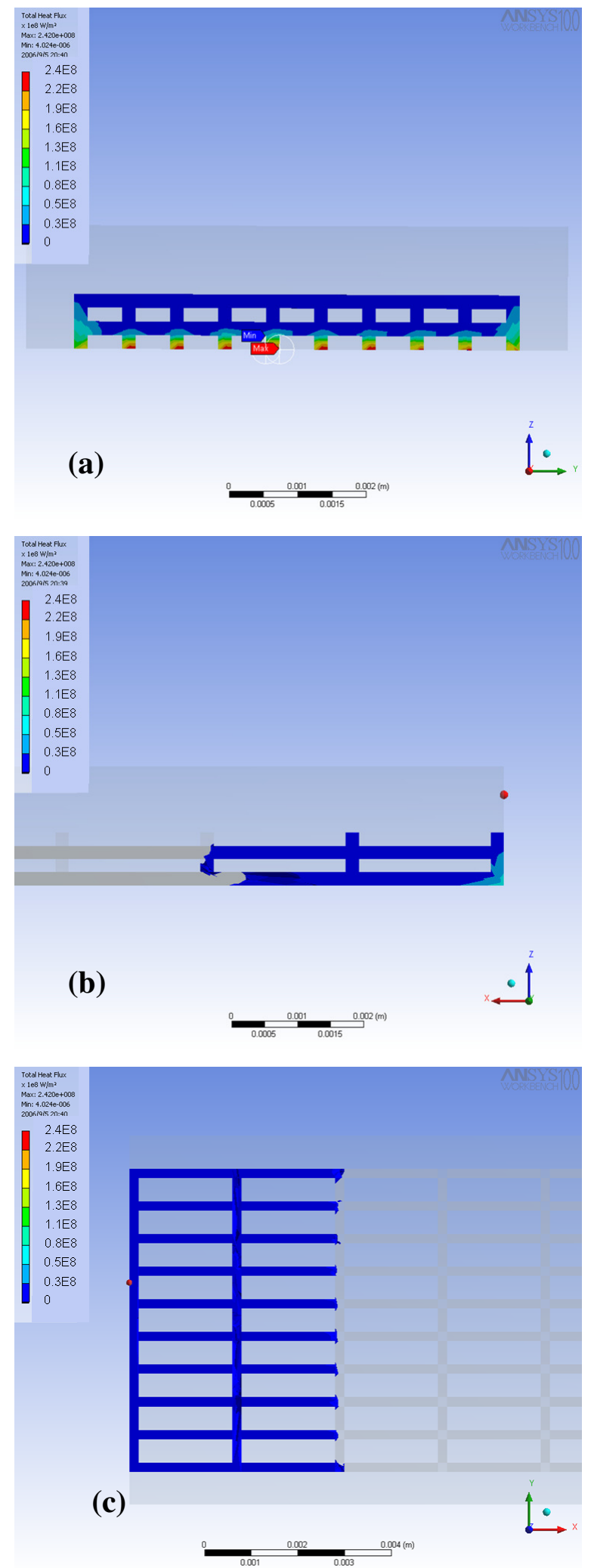

Figure 4-16: Total heat flux in $\mathrm{W} / \mathrm{m}^{2}$, a) front view, b) side view, and c) top view 

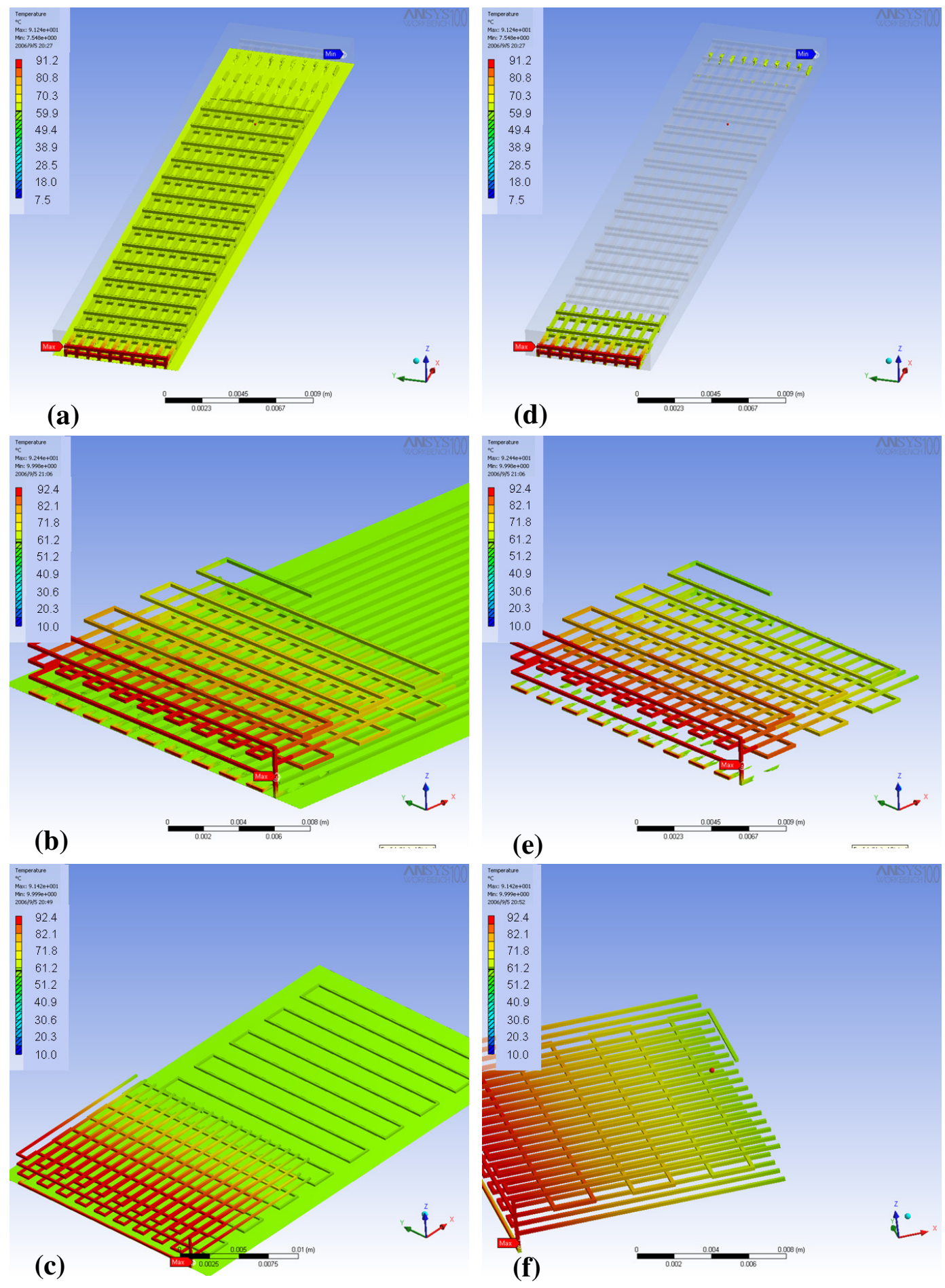

Figure 4-17: Temperature contour in ${ }^{\circ} \mathrm{C}$ for $T>59.9^{\circ} \mathrm{C}$ for $\mathbf{a}$ ) case $1, \mathbf{b}$ ) case 2 , and c) case 3 ; Temperature contour ${ }^{\circ} \mathrm{C}$ for $T>60^{\circ} \mathrm{C}$ for d) case 1, e) case 2 , and f) case 3 


\subsection{Two-phase model}

Based on the results of the three-dimensional investigation, the interconnected network was selected to consider the effect of two-phase flow. The basic idea of employing three-dimensional network of microchannel was evaluated here to demonstrate if the three-dimensional geometry can improve the thermal characteristics of a passive microfluidic chip. Unlike the two-phase flow in micro modelling approach, in the current three-dimensional investigation, a phase separation is expected in the microchannels. The circulation of the two-phase flow requires the bottom layers of microchannel to contain liquid water, whereas the top layers transfer vapour. Similar to the two-phase flow simulation in the micro modelling section, water was selected as working fluid.

Figure 4-18 shows the geometry and dimensions of the interconnected network of microchannels. Square cross-section with sides of $200 \mu \mathrm{m}$ was selected, while a length of $21.8 \mathrm{~mm}$ was chosen for the microfluidic chip. In order to investigate the flow characteristics in the network, a representative plane in the middle of the microfluidic chip $(y=1.5 \mathrm{~mm})$ was considered. The location of the representative plane is shown in Figure 4-18.

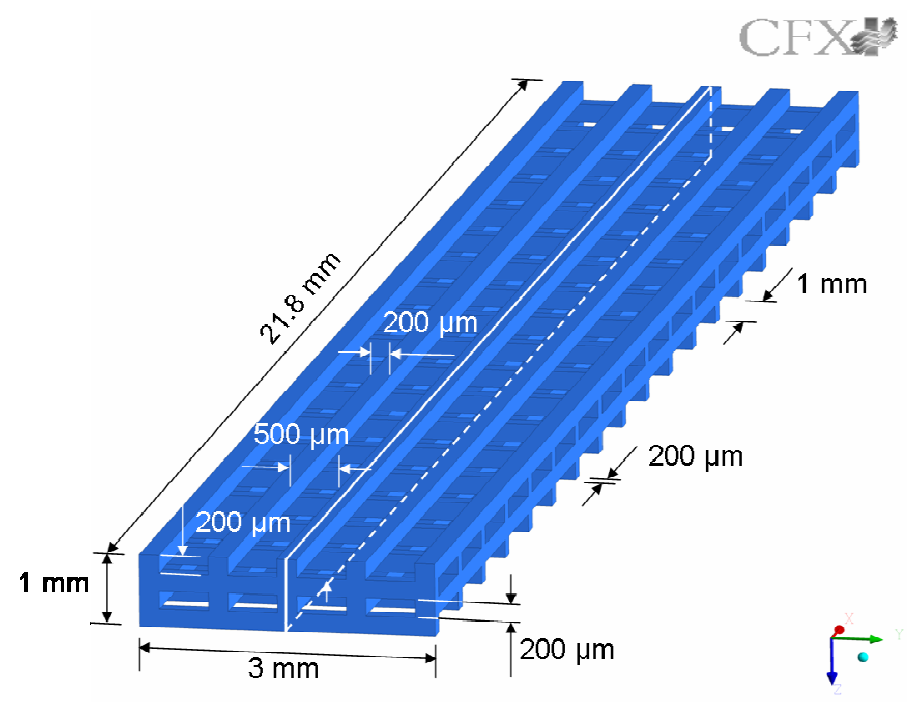

Figure 4-18: Microchannels geometry and dimensions. The white lines determine the place of the representative plane. 
ANSYS CFX-5.7.1 finite element software was employed for the current analysis. Figure 4-19 illustrates the microchannel meshing. The model was meshed with 91000 elements. A maximum spacing of $80 \mu \mathrm{m}$ was selected for the elements. Due to the square cross-section for the microchannels, the connections between the layers enable the working fluid to flow from one layer to another one. Considering the entire network as one-block benefits the meshing to be identical in the connections of layers.

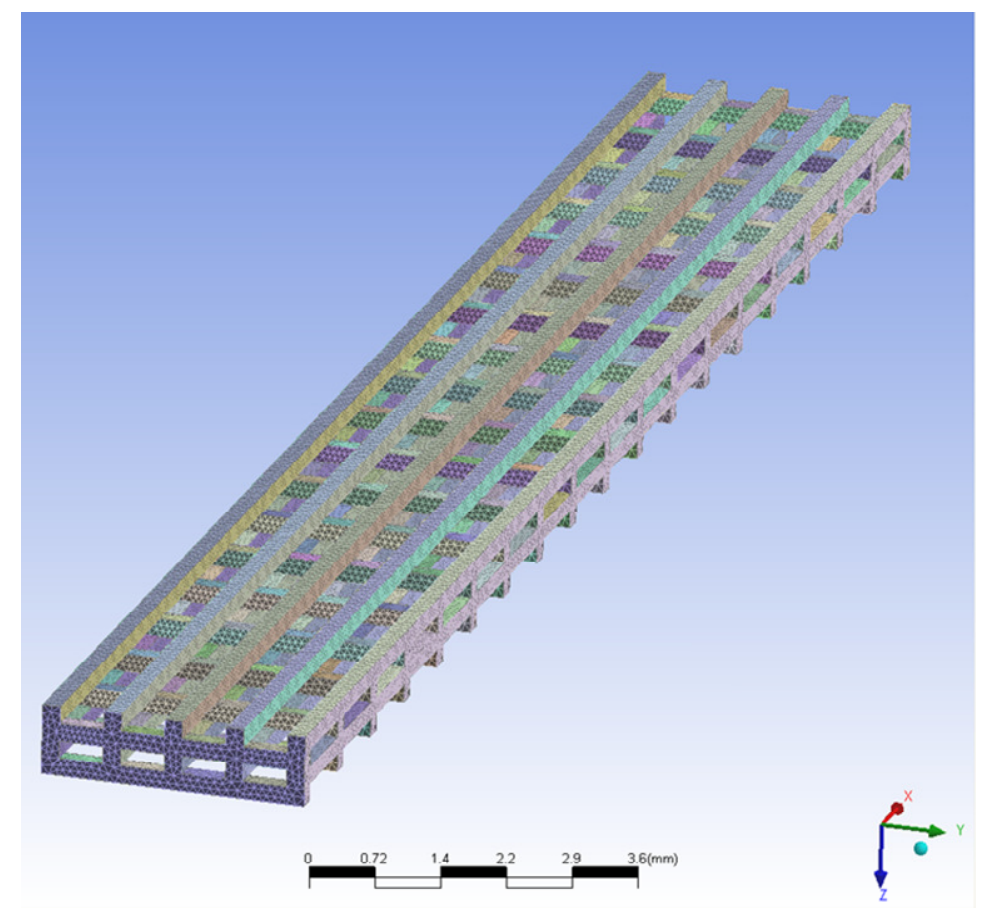

Figure 4-19: Microchannels meshing for the two-phase flow in three-dimensional geometry

Table 4-2 lists the different analyses performed in this section. Two sets of boundary conditions were applied to the models: fixed temperature and applied heat flux. Two sets of liquid filling ratios were set as initial conditions, 25 and $75 \%$.

Figure 4-20 shows the location of boundary conditions in the model. Hot source and cold sink boundary conditions were applied at the ends of microfluidic chip. 
For example for Run 1, which is under temperature boundary conditions, the cold sink and hot source temperatures are $30^{\circ} \mathrm{C}$ and $70^{\circ} \mathrm{C}$, respectively. However, for Run 2, which is under heat flux boundary conditions, the heat flux in evaporator and condenser are $+1 \mathrm{~kW} / \mathrm{m}^{2}$ and $-1 \mathrm{~kW} / \mathrm{m}^{2}$, respectively.

Table 4-2: Different model setup for two-phase flow in three-dimensional geometry

\begin{tabular}{cccccc}
\hline \multirow{2}{*}{ Run } & Filling ratio & \multicolumn{2}{c}{ Boundary Condition } & \multirow{2}{*}{ Time $(\mathbf{s})$} \\
\cline { 3 - 4 } & $(\%)$ & Type & Hot & Cold & \\
\hline 1 & 25 & Temperature $\left({ }^{\circ} \mathrm{C}\right)$ & 70 & 30 & 30 \\
2 & 75 & Heat flux $\left(\mathrm{kW} / \mathrm{m}^{2}\right)$ & +1 & -1 & 30 \\
3 & 75 & Temperature $\left({ }^{\circ} \mathrm{C}\right)$ & 50 & 40 & 30 \\
4 & 25 & Heat flux $\left(\mathrm{kW} / \mathrm{m}^{2}\right)$ & +10 & -10 & 30 \\
5 & 25 & Temperature $\left({ }^{\circ} \mathrm{C}\right)$ & 90 & 5 & 30 \\
\hline
\end{tabular}

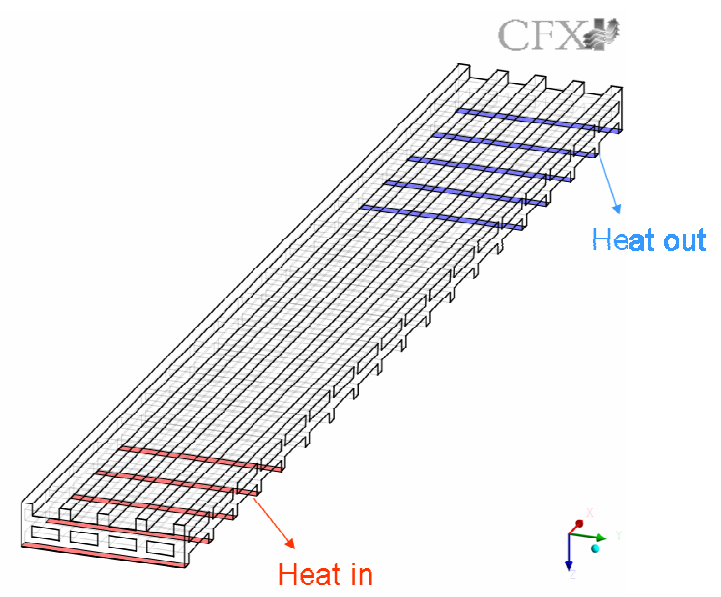

Figure 4-20: Microchannels boundary conditions for two-phase flow in three-dimensional geometry

Most of the software setups for the current three-dimensional analysis were similar to those of the two-phase flow analysis in the previous chapter (section 3.3 ) for example the same surface tension coefficient of $0.073 \mathrm{~N} / \mathrm{m}$ was applied to the models. Based on the software instructions, no slip boundary condition was applied for the liquid phase, while slip boundary condition was employed for the vapor flow. In order to compromise the run time and the accuracy of the results, a 
maximum number of 200 iterations with the residual target of $1 \mathrm{E}-4$ were applied to the simulation.

The main factor that defines the proper operation of the current microfluidic chip is the circulation of two phases in the microchannels. Figure 4-21 shows the water/water vapour interphase mass transfer, which is an index of phase change in the microfluidic chip. The results are presented for the representative plane (see Figure 4-18). The left side of the contour represents the evaporator section (i.e. the hot zone), and the right hand end of the contour illustrates the condenser section (i.e. the cold zone). A clockwise circulation of the two phases is observed. In the evaporator section (left side), the evaporation process takes place in the top layers. Then, the vapour flows towards the condenser section through the top microchannels. In the condenser section (right side), the condensation process happens in the bottom layers and the liquid phase returns to the evaporator through the bottom layers of the microchannels network. The steady state circulation of the phases within the microchannels demonstrates the proper operation of the microfluidic chip.

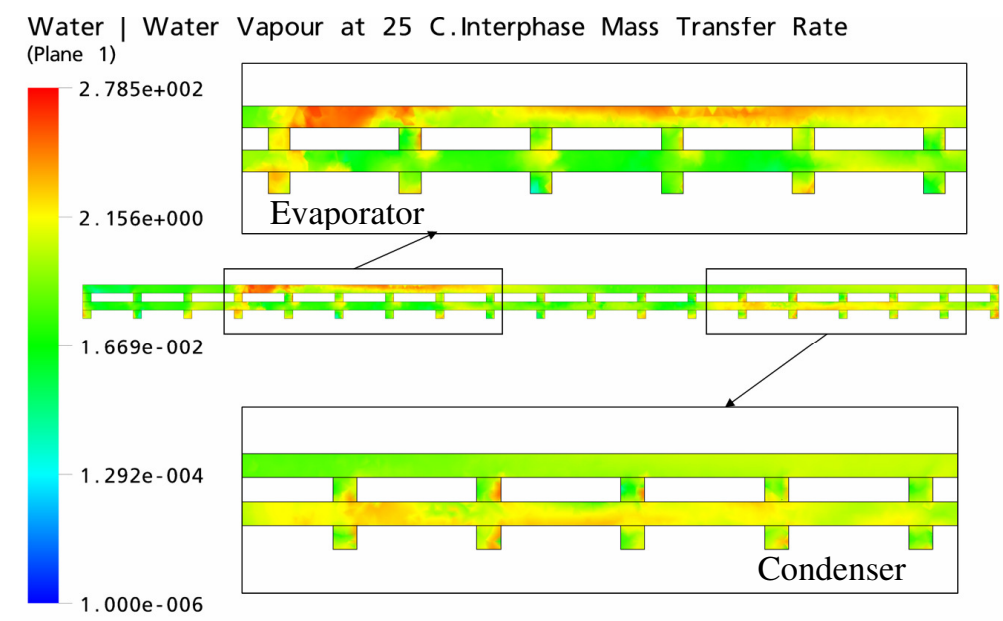

$\left[\mathrm{kg} \mathrm{s}^{\wedge}-1 \mathrm{~m}^{\wedge}-3\right]$

Figure 4-21: Water/water vapour interphase mass transfer rate $\left(\mathrm{kg} / \mathrm{m}^{3} \mathrm{~s}\right)$ in the representative plane for Run 5 


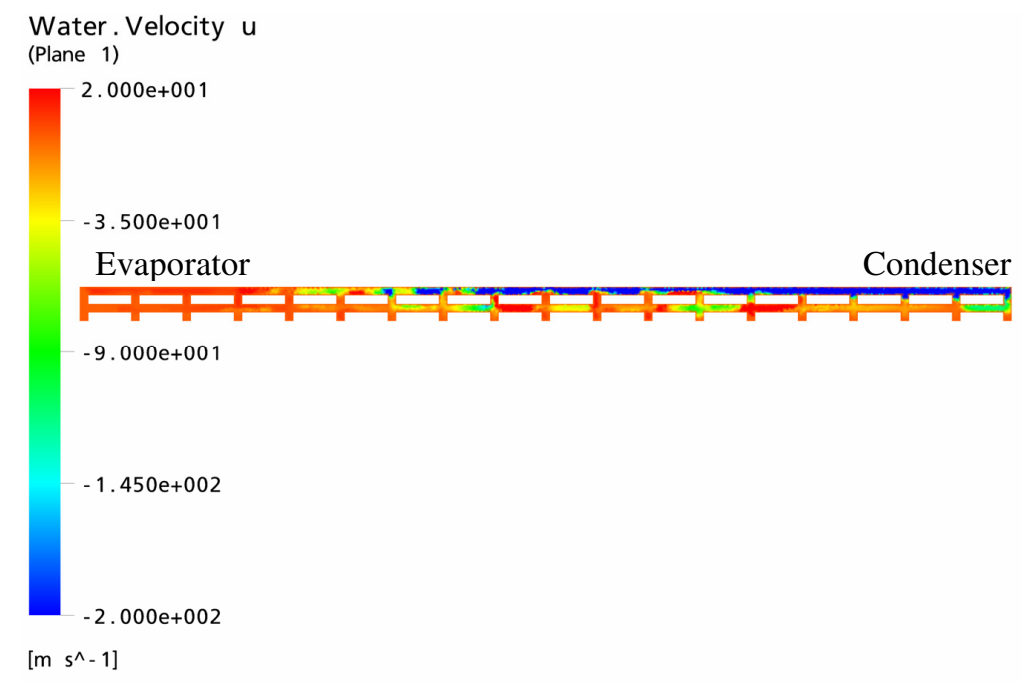

Figure 4-22: Water axial velocity $(\mathrm{m} / \mathrm{s})$ in the representative plane for Run 1

The flow circulation in the microchannels can also be demonstrated through the axial velocity of the fluids. Figure 4-22 illustrates the axial velocity of the water through the channels, in the representative plane. The positive direction is from the right to the left, so the negative values (the blue color) demonstrate a flow from the left to the right. The concept of flow circulation is evident in Figure $4-22$.

The two-phase analysis in the ANSYS CFX software allocates an initial ratio of liquid and vapour phases to the investigation field; for instance, in Run 1 of the analyses (see Table 4-2) the initial volume fraction of liquid water in the entire network of microchannels is equal to $25 \%$. In the steady state conditions, the distribution of liquid and vapour phases in the microchannels can indicate the operation of the microfluidic chip. Figure 4-23 shows the vapour volume fraction in the representative plane, for Run 1. Most of the regions are filled with the red color that illustrates the volume fractions close to unity. Since the density of the vapour phase is much lower than that of liquid phase, in case of equal masses, the volume of the vapour phase is larger than the volume of the liquid. Therefore it is expected to have high volume fractions of vapour in most regions of the microchannels network. According to the definition of volume fraction in two- 
phase flows, the summation of liquid volume fraction and vapour volume fraction in an arbitrary point must be equal to unity. Thus, it is expected that in the regions where the vapour volume fraction is closed to zero, the liquid volume fraction be close to unity. Based on the difference in the densities of the two-phases, the blue zones in Figure 4-23 are expected to be fully occupied by the liquid phase. The presence of liquid phase in the evaporator section (left side of the contour) demonstrated that the dry-out phenomenon has not occurred and the microfluidic chip has not yet overloaded. The low initial volume fraction of liquid water in Run 1 (i.e. 25\%) led to have small zone of liquid phase in Figure 4-23. The concentration of liquid phase in the condenser section (right side of the contour) demonstrates the condensation process in that zone.

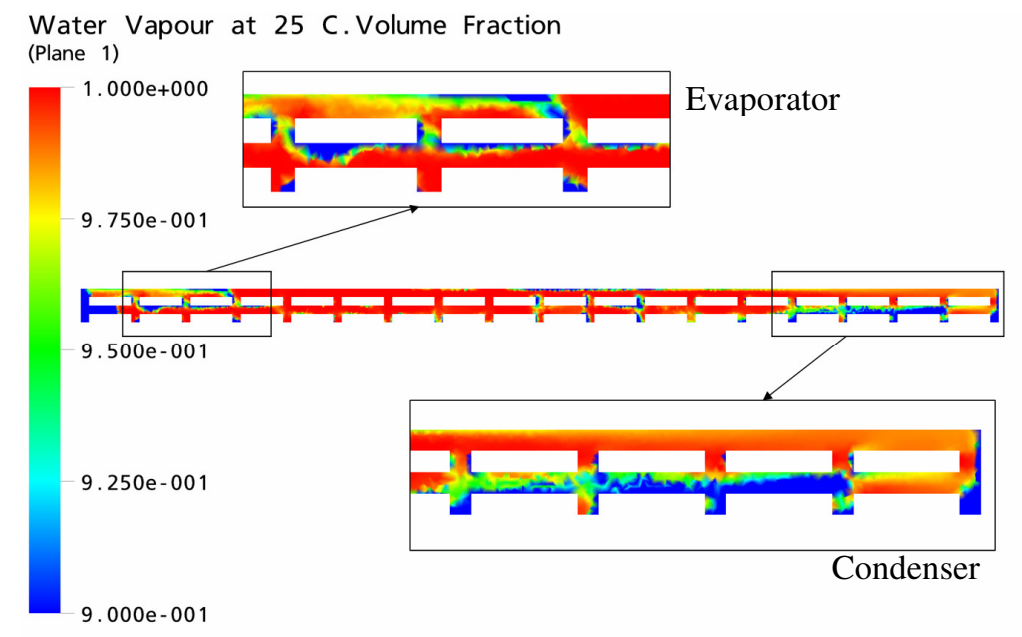

Figure 4-23: Vapour volume fraction in the representative plane for Run 1

Figure 4-24 illustrates the vapour conservative volume fraction. It is clear that the conservative volume fraction for the vapour phase decreases from the evaporator to the condenser (from the left side of the contour to the right). Two factors are involved in the decrease of vapour conservative volume fraction: temperature effect and phase change effect. First, the temperature decreases from the evaporator towards the condenser; for example for Run 1, there is a $40^{\circ} \mathrm{C}$ temperature difference between the evaporator and condenser. Furthermore, as 
mentioned in the previous chapter, the conservative volume fraction is an index of density. Therefore, decreasing the temperature towards the condenser, leads to the decrease in the density or conservative volume fraction. Secondly, the phase change issues affect the decrease of the conservative volume fraction of vapour phase towards the condenser. When the mixture of liquid and vapour phases flows on the way to the condenser, phase change process takes place, and the condensation decreases the conservative volume fraction of the mixture.

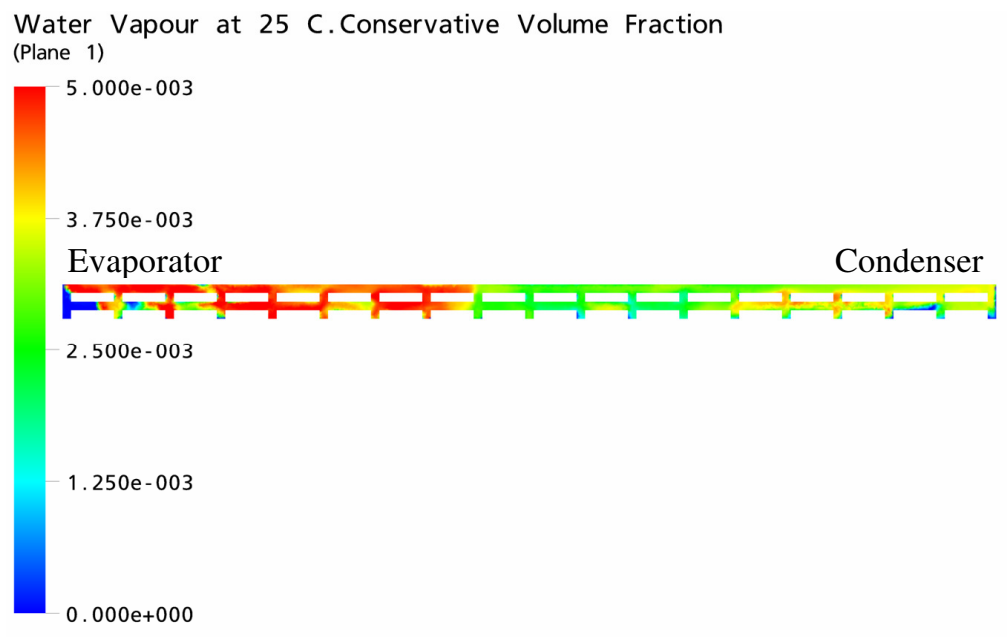

Figure 4-24: Vapour conservative volume fraction in the representative plane for Run 1

Finally, the temperature gradient within the entire network of microchannels is of particular interests. Figure 4-25 shows the vapour temperature contour for Run 3. The $10^{\circ} \mathrm{C}$ temperature difference between the evaporator and condenser is shown in this figure. This microfluidic chip is operating properly, because the temperature gradient is occurred within the entire length of the microfluidic chip. Comparing the results for Figure 4-25 and Figure 4-26 demonstrates the overloading conditions for Run 4. Figure 4-25 shows that the penetration of hot temperatures (distinguished by red color) in the longitudinal direction of the network is limited, so the maximum thermal capacity of the microfluidic chip is higher than that. However, for Run 4 the long penetration of the hot temperature in the longitudinal direction of the network led to very small temperature gradient 
along the microchannels, indicating an overloading condition. In this case, the condensed water (or even cold vapour) could not go far back towards the evaporator, and therefore the effective length of the network was much less than the physical length of the microfluidic chip. Thus, the microfluidic chip was not able to transport heat to the cold sink, and the thermal load on the chip was superior to the maximum capacity of the microfluidic chip.

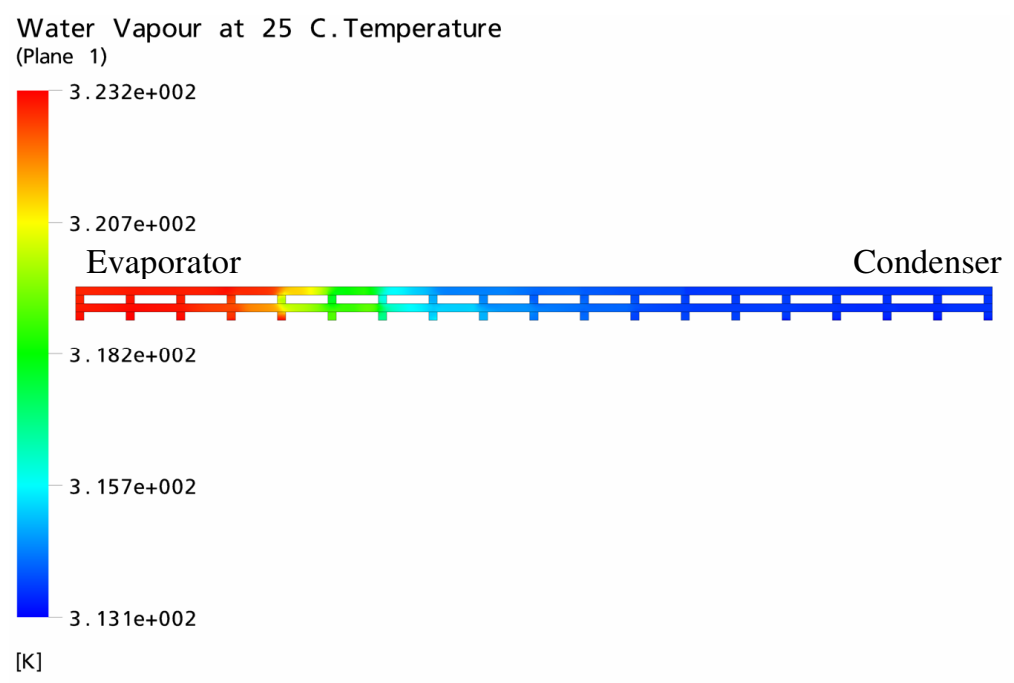

Figure 4-25: Vapour temperature contour $(\mathrm{K})$ in the representative plane for Run 3

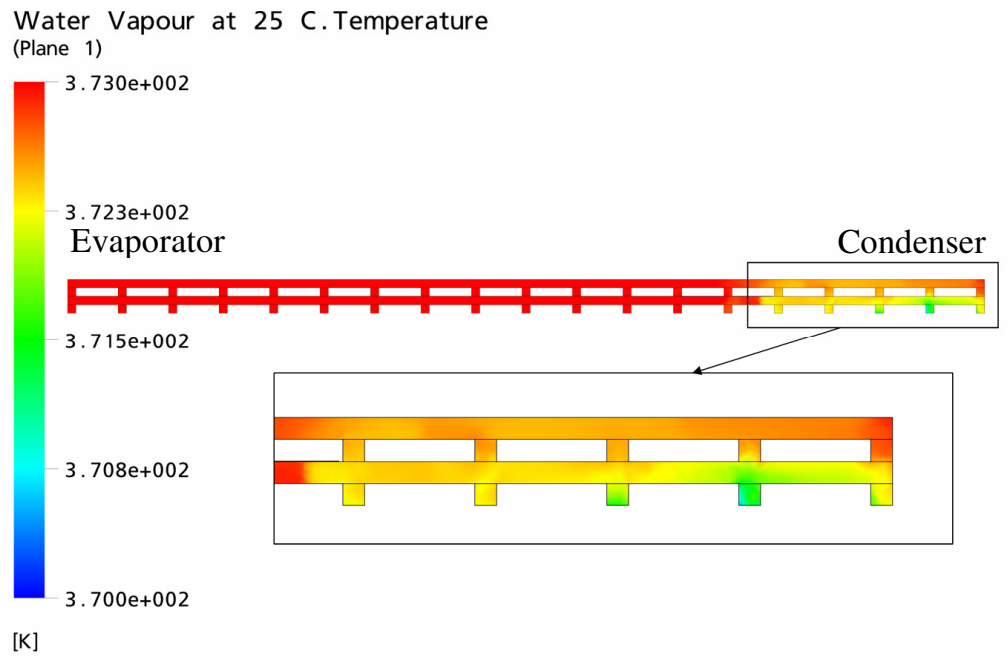

Figure 4-26: Vapour temperature contour $(\mathrm{K})$ in the representative plane for Run 4 


\section{Chapter 5 Conclusion}

\subsection{Contribution}

In the current thesis, using two strategies, the effect of geometry on the heat transfer efficiency of microfluidic chips was investigated. A decoupling approach was employed to consider the micro and macro models, separately. In addition, the fluid flow/heat transfer problem was simulated for a single micro heat pipe and a three-dimensional network of microchannels. The following conclusions can be drawn:

The effect of boundary conditions was investigated for single microchannel. A single microchannel was simulated and it was concluded that by ignoring the reorientation zone, the boundary conditions could be simplified.

The microchannel cross-section was optimized for single-phase flows. It was shown that circular cross-section had the highest thermal performance. However, the lack of sharp corners, reduces the capillary pressure difference in these channels, and limits the application to active microfluidic chips.

Two-phase flow in single triangular microchannels was investigated. The phenomenon of liquid accumulation in the sharp corners was accurately captured in the model, and the phase change process within the microchannel was successfully modeled. The driving pressure difference between the evaporator and condenser, the effective thermal conductivity, and the velocity field were compared with the literature and a good agreement was observed. The two-phase investigation was also expanded to simulate the overloading conditions.

Geometric optimization of arrays of microchannels in the macro modelling section was performed. A planar parametric model was built. Triangular, rectangular and trapezoidal microchannels were investigated in the single-phase 
model. Since the optimization process of microfluidic chips depends on the situations in which the chip should operate.

\section{General design charts were presented in order to enable the designer to select} the optimum geometry. The transient behaviour of the microfluidic chip under a high rate ramp of the evaporator temperature showed that a very short time of $2 \mathrm{~s}$ was needed to reach the steady state condition.

\section{A two-phase flow simulation in the interconnected three-dimensional geometry was conducted. Two-phase circulation of the working fluid demonstrated the proper operation of the micro heat pipe. The overloading situation was also examined and the system behaviour was fully simulated.}

\subsection{Future work}

Further studies in the field of geometric optimization for thermal microfluidic chips can be classified into two categories: modelling investigations, and validation studies.

The future modelling issues worth addressing include:

- Modelling of more complex geometries (e.g. polygonal, curved sided, or combined cross-sections, as well as interconnected geometries).

- Geometric optimization of three-dimensional networks, using two-phase flow simulations. In the current thesis, the optimum geometry was achieved through a single-phase approach. While, two-phase simulation demonstrated the proper operation and accurately captured the detailed phenomena. It should be mentioned that each run of a two-phase model lasted around 8 hours on a personal computer. 
- Transient analysis on a two-phase model. Due to the fluid dynamics issues, it is expected that the system needs more than $2 \mathrm{~s}$ in order to converge to the steady state condition.

- A combined two-phase model with the channels embedded inside an epoxy block. There are some difficulties in defining the boundary conditions for the microchannels' walls.

The validation phase of the current work may include:

- Experimental investigation on a single micro heat pipe manufactured by direct-write method.

- Experimental analysis on an array of micro heat pipes in order to validate the design charts presented in Chapter 4.

- Experimental validation of the three-dimensional network operation.

- Theoretical investigation on the micro heat pips transient operation as explained in Appendix A. 


\section{References}

1. Webb, R.L. and N.-H. Kim, Principles of enhanced heat transfer. Second ed. 2005, New York: Taylor \& Francis Group. 795.

2. Cengel, Y.A., Heat Transfer: a practical approach. Second ed. 2003, New York: McGraw-Hill.

3. Launay, S., V. Sartre, and M. Lallemand, Experimental study on silicon micro-heat pipe arrays. Applied Thermal Engineering, 2004. 24(2-3): p. 233.

4. Tuckerman, D.B. and R.F.W. Pease, High-performance heat sinking for VLSI. IEEE Electron Device Letters, 1981. ED-2(5): p. 126.

5. Tien, C.-L., A. Majumdar, and F.M. Gerner, Microscale energy transport. Series in chemical and mechanical engineering. 1998, Washington, DC: Taylor \& Francis.

6. Zohar, Y., Heat convection in micro ducts. Microsystems, ed. S. Senturia. 2003, Norwell, Massachusetts: Kluwer Academic Publishers. 202.

7. Kandlikar, S.G., et al., Heat transfer and fluid flow in minichannels and microchannels. 2006, Oxford: Elsevier Ltd. 450.

8. Zhang, L., T.W. Kenny, and K.E. Goodson, Silicon microchannel heat sinks. Microtechnology and MEMS, ed. H. Baltes, H. Fujita, and D. Liepmann. 2004, New York: Springer-Verlag Berlin Heidelberg. 140.

9. Sobhan, C.B. and S.V. Garimella, A comparative analysis of studies on heat transfer and fluid flow in microchannels. Microscale Thermophysical Engineering, 2001. 5(4): p. 293311.

10. Cotter, T.P. Principles and prospects for micro heat pipes. in 5th International Heat Pipe Conference. 1984. Tsukuba, Japan.

11. Peterson, G.P., An introduction to heat pipes: modeling, testing, and applications. 1994, New York: John Wiley \& Sons, Inc. 356.

12. Faghri, A., Heat pipe science and technology. 1995, Washington, DC: Taylor \& Francis.

13. Babin, B.R., G.P. Peterson, and D. Wu, Steady-state modeling and testing of a micro heat pipe. Journal of Heat Transfer, Transactions ASME, 1990. 112(3): p. 595.

14. Peterson, G.P., Overview of micro heat pipe research and development. Applied Mechanics Reviews, 1992. 45(5): p. 175.

15. Peterson, G.P., Modeling, fabrication, and testing of micro heat pipes: An update. Applied Mechanics Reviews, 1996. 49(10 pt 2): p. 175-183.

16. Kang, S.-W., S.-H. Tsai, and M.-H. Ko, Metallic micro heat pipe heat spreader fabrication. Applied Thermal Engineering, 2004. 24(2-3): p. 299.

17. Tzanova, S., et al. Analytical investigation of flat silicon micro heat spreaders. 2004. Seattle, WA, United States: Institute of Electrical and Electronics Engineers Inc., Piscataway, NJ 08855-1331, United States. 
18. Kalahasti, S. and Y.K. Joshi, Performance characterization of a novel flat plate micro heat pipe spreader. IEEE Transactions on Components and Packaging Technologies, 2002. 25(4): p. 554.

19. Riffat, S.B., X. Zhao, and P.S. Doherty, Analytical and numerical simulation of the thermal performance of 'mini' gravitational and 'micro' gravitational heat pipes. Applied Thermal Engineering, 2002. 22(9): p. 1047.

20. Ghajar, M., J. Darabi, and N. Crews Jr, A hybrid CFD-mathematical model for simulation of a MEMS loop heat pipe for electronics cooling applications. Journal of Micromechanics and Microengineering, 2005. 15(2): p. 313.

21. Liepmann, D. Design and fabrication of a micro-CPL for chip-level cooling. 2001. New York, NY, United States: American Society of Mechanical Engineers, New York, NY 10016-5990, United States.

22. Ghajar, M. and J. Darabi, Numerical modeling of evaporator surface temperature of a micro loop heat pipe at steady-state condition. Journal of Micromechanics and Microengineering, 2005. 15(10): p. 1963.

23. Ha, J.M. and G.P. Peterson, The heat transport capacity of micro heat pipes. Transactions of the ASME. Journal of Heat Transfer, 1998. 120(4): p. 1064.

24. Longtin, J.P., B. Badran, and F.M. Gerner. One-dimensional model of a micro heat pipe during steady-state operation. 1992. San Diego, CA, USA: Publ by ASME, New York, NY, USA.

25. Stephan, P.C. and C.A. Busse, Analysis of the heat transfer coefficient of grooved heat pipe evaporator walls. International Journal of Heat and Mass Transfer, 1992. 35(2): p. 383.

26. Ma, H.B. and G.P. Peterson, Temperature variation and heat transfer in triangular grooves with an evaporating film. Journal of Thermophysics and Heat Transfer, 1997. 11(1): p. 90 .

27. Sartre, V., M.C. Zaghdoudi, and M. Lallemand, Effect of interfacial phenomena on evaporative heat transfer in micro heat pipes. International Journal Thermal Science, 2000. 39: p. 498-504.

28. Tiselj, I., et al., Effect of axial conduction on the heat transfer in micro-channels. International Journal of Heat and Mass Transfer, 2004. 47(12-13): p. 2551.

29. Khrustalev, D. and A. Faghri, Thermal analysis of a micro heat pipe. Journal of Heat Transfer, Transactions ASME, 1994. 116(1): p. 189.

30. Peterson, G.P. and H.B. Ma, Theoretical analysis of the maximum heat transport in triangular grooves: a study of idealized micro heat pipes. Transactions of the ASME. Journal of Heat Transfer, 1996. 118(3): p. 731.

31. Wu, D. and G.P. Peterson, Investigation of the transient characteristics of a micro heat pipe. Journal of Thermophysics and Heat Transfer, 1991. 5(2): p. 129.

32. Peterson, G.P. and H.B. Ma. Temperature response and heat transport in a micro heat pipe. 1998. Albuquerque, NM, USA: ASME, Fairfield, NJ, USA. 
33. Ha, J.M. and G.P. Peterson, Analytical prediction of the axial dryout point for evaporating liquids in triangular microgrooves. Journal of Heat Transfer, Transactions ASME, 1994. 116(2): p. 498.

34. Peterson, G.P., et al., Analytical and experimental investigation of the rewetting of circular channels with internal V-grooves. International Journal of Heat and Mass Transfer, 1992. 35(11): p. 3085.

35. Schneider, M., M. Yoshida, and M. Groll. Investigation of interconnected mini heat pipe arrays for micro electronics cooling. in 11th International Heat Pipe Conference. 1999. Tokyo, Japan.

36. Suman, B., S. De, and S. DasGupta, A model of the capillary limit of a micro heat pipe and prediction of the dry-out length. International Journal of Heat and Fluid Flow, 2005. 26(3): p. 495.

37. Suman, B. and P. Kumar, An analytical model for fluid flow and heat transfer in a microheat pipe of polygonal shape. International Journal of Heat and Mass Transfer, 2005. 48(21-22): p. 4498.

38. Suman, B. and N. Hoda, Effect of variations in thermophysical properties and design parameters on the performance of a V-shaped micro grooved heat pipe. International Journal of Heat and Mass Transfer, 2005. 48(10): p. 2090.

39. Dickey, J.T. and T.T. Lam. Impact of channel geometry on heat transfer in microchannel for high density electronics cooling. 2001. Kauai, Hi, United States: American Society of Mechanical Engineers, New York, NY 10016-5990, United States.

40. Li, J. and G.P. Peterson, Geometric optimization of a micro heat sink with liquid flow. IEEE Transactions on Components and Packaging Technologies, 2006. 29(1): p. 145.

41. Suman, B., S. De, and S. DasGupta, Transient modeling of micro-grooved heat pipe. International Journal of Heat and Mass Transfer, 2005. 48(8): p. 1633.

42. Peterson, G.P., A.B. Duncan, and M.H. Weichold, Experimental investigation of micro heat pipes fabricated in silicon wafers. Journal of Heat Transfer, Transactions ASME, 1993. 115(3): p. 751.

43. Chung, P.M.Y., et al., Two-Phase Flow Through Square and Circular Microchannels--Effects of Channel Geometry. Journal of Fluids Engineering, 2004. 126(4): p. 546.

44. Lee, M., M. Wong, and Y. Zohar, Characterization of an integrated micro heat pipe. Journal of Micromechanics and Microengineering, 2003. 13(1): p. 58.

45. Zhang, L., et al., Phase change phenomena in silicon microchannels. International Journal of Heat and Mass Transfer, 2005. 48(8): p. 1572.

46. Hetsroni, G., et al., A uniform temperature heat sink for cooling of electronic devices. International Journal of Heat and Mass Transfer, 2002. 45(16): p. 3275.

47. Ma, H.B. and G.P. Peterson, Experimental investigation of the maximum heat transport in triangular grooves. Transactions of the ASME. Journal of Heat Transfer, 1996. 118(3): p. 740.

48. Moon, S.H., et al., Experimental study on the thermal performance of micro-heat pipe with cross-section of polygon. Microelectronics Reliability, 2004. 44(2): p. 315. 
49. Seok Hwan, M., et al., Improving thermal performance of miniature heat pipe for notebook PC cooling. Microelectronics Reliability, 2002. 42(1): p. 135.

50. Duncan, A.B. and G.P. Peterson, Charge optimization for a triangular-shaped etched micro heat pipe. Journal of Thermophysics and Heat Transfer, 1995. 9(2): p. 365.

51. Kang, S.-W., S.-H. Tsai, and H.-C. Chen, Fabrication and test of radial grooved micro heat pipes. Applied Thermal Engineering, 2002. 22(14): p. 1559.

52. Wu, D., G.P. Peterson, and W.S. Chang, Transient experimental investigation of micro heat pipes. Journal of Thermophysics and Heat Transfer, 1991. 5(4): p. 539.

53. Tabeling, P., Introduction to microfluidics. 2005, New York: Oxford university press. 297.

54. Liu, C.W., C. Gau, and B.T. Dai, Design and fabrication development of a micro flow heated channel with measurements of the inside micro-scale flow and heat transfer process. Biosensors and Bioelectronics, 2004. 20(1): p. 91.

55. Therriault, D., S.R. White, and J.A. Lewis, Chaotic mixing in three-dimensional microvascular networks fabricated by direct-write assembly. Nature Materials, 2003. 2(4): p. 265.

56. Therriault, D., et al., Fugitive inks for direct-write assembly of three-dimensional microvascular networks. Advanced Materials, 2005. 17(4): p. 395.

57. Palm, S., et al. Micro-heat pipe triangular cross section by direct-write. in Third Microfluidics French Conference. 2006. Toulouse, France.

58. Bey-Oueslati, R., et al. High speed direct-write for rapid fabrication of three-dimensional microfluidic devices. in Third Microfluidics French Conference. 2006. Toulouse, France.

59. Rahmat, M. and P. Hubert. Numerical simulation for the geometric design of micro heat pipes. in Third Microfluidics French Conference. 2006. Toulouse, France.

60. Bryning, M.B., et al., Thermal conductivity and interfacial resistance in single-wall carbon nanotube epoxy composites. Applied Physics Letters, 2005. 87(16): p. 161909.

61. Kimura, T., et al. Thermal conductivity and RF signal transmission properties of Agfilled epoxy resin. 2003. New Orleans LA, United States: Institute of Electrical and Electronics Engineers Inc.

62. $\mathrm{Xu}, \mathrm{Y}$. and D.D.L. Choung, Increasing the thermal conductivity of boron nitride and aluminum nitride particle epoxy-matrix composites by particle surface treatments. Composite Interfaces, 2000. 7(4): p. 243-256.

63. Motoyama, T. and R. Hackam. Effect of temperature and water salinity on hydrophobicity of epoxy resin. 2001. Kitchener, ON: Institute of Electrical and Electronics Engineers Inc.

64. Nicolas, R., Fabrication de microcaloducs par écriture directe. 2007, McGill University: Montreal. p. 35 .

65. Tsai, N.-C. and C.-Y. Sue, SU-8 based continuous-flow RT-PCR bio-chips under highprecision temperature control. Biosensors and Bioelectronics, 2006. 22(2): p. 313. 


\section{Appendix A Governing equations}

\section{Assumption}

1- Both liquid and vapour flows are incompressible. The Mach number of two flows is much less than 1 , and the incompressibility assumption is valid.

2- Surface tension is constant.

3- At any time, the vapour pressure is independent of position and assumed to be the vapour saturation pressure at that temperature.

4- Enthalpy and internal energy are only linear functions of temperature.

5- There is no heat transfer at the adiabatic section.

6- Density of liquid and vapour are assumed to be constant.

7- Tilt angle is constant.

8- Contact angle is zero.

\section{Boundary conditions}

1- At $x=0$ and $x=L: V_{l}=V_{v}=0$

2- $T_{\text {inf }}$ is a inear or Step function of time

3- At $x=0: r=0$

4- $P_{v}$ is not a function of position

5- $T_{\text {inf }}$ is uniform at different sections of evaporator, condenser and adiabatic.

6- At $x=L: r=r_{r e f}$ and $P_{l}=P_{v}-\sigma / r_{r e f}$

7- $r_{\text {ref }}$ can be found from the geometry

\section{Initial conditions}

1- At $t=0: V_{l}=V_{v}=0$

2- At $t=0: T_{l}=T_{v}=T_{\text {inf }}$

3- At $t=0: P_{v}=P_{\text {sat }}$ at $T_{\text {inf }}$

4- At $t=0: P_{l}=P_{\text {sat }}-\left(\sigma / r_{\text {ref }}\right)-\rho l g z \sin \psi$

5- At $t=0: r=\sigma /\left(P_{\text {sat }}-P_{l}\right)$ 


\section{Derivation}

Figure A-1 shows a schematic of the micro heat pipe cross-section with the parameters used in the formulation.

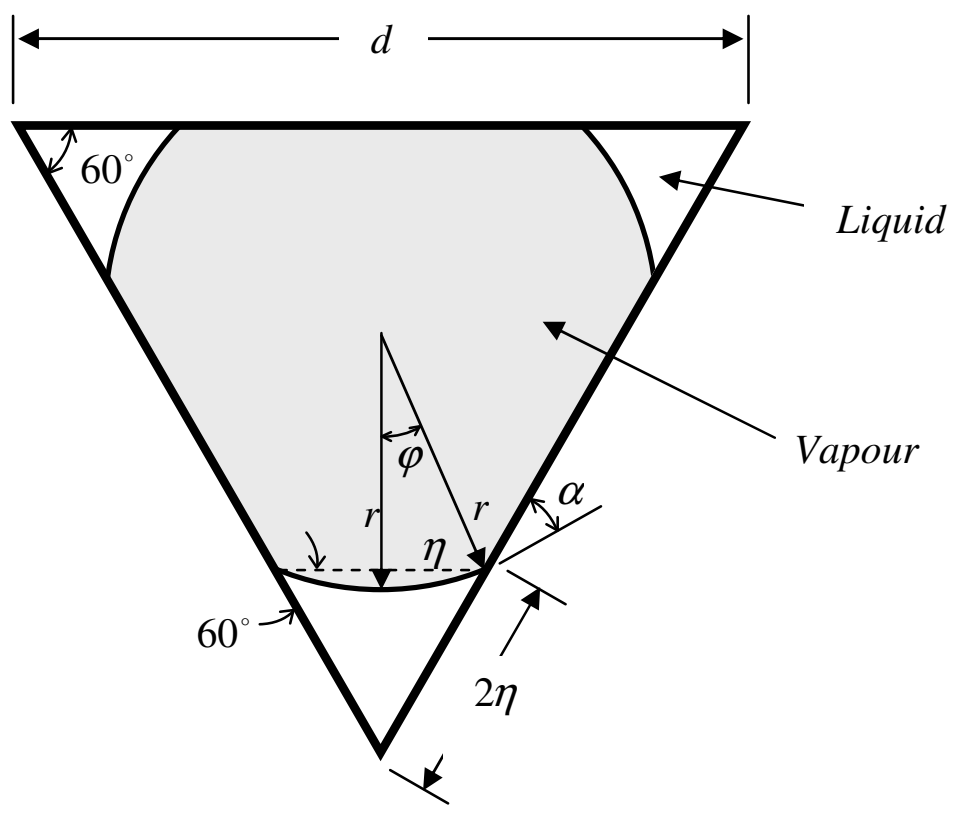

Figure A-1: Micro heat pipe cross-section

A transient one-dimensional (along the microchannel length) analysis is formulated, here. The main objective of the formulation is determining six parameters as functions of time and position in the micro heat pipe. These six parameters are including velocity and temperature for both liquid and vapour phases, and pressure for the liquid phase, as well as the radius of curvature in the interface of liquid and vapour phases. Therefore, six independent equations are required to define the mentioned parameters. These six equations are LaplaceYoung equation, liquid pressure drop along the channel, conservation of momentum for liquid and vapour, and conservation of energy for liquid and vapour. Fortunately, only the first four equations are coupled together and include four parameters. From the first four equations, liquid and vapour velocity, as well as liquid pressure and interface radius of curvature can be defined. Then, based on these results, the phase change heat flux can be calculated. The last two equations 
are conservation of energy for liquid and vapour phases. Importing all data into these equations lead to obtain the temperature in liquid and vapour phases.

First of all, it is required to define the geometric parameters that are used in the following formulation. From Longtin et al. [24] the cross-sectional area for liquid and vapour, as well as the interface area of liquid, vapour, and the wall can be defined.

$$
\begin{aligned}
& A_{l}=\beta_{l} r^{2} \\
& A_{v}=\frac{\sqrt{3}}{4} d^{2}-\beta_{l} r^{2} \\
& A_{l, w}=\beta_{l, w} r d z \\
& A_{v, w}=\left(3 d-\beta_{l, w} r\right) d z \\
& A_{i}=\beta_{i} r d z
\end{aligned}
$$

where

$$
\begin{aligned}
& \beta_{l}=3\left[\sqrt{3} \sin ^{2}\left(\frac{\pi}{3}-\alpha\right)+\frac{1}{2} \sin 2\left(\frac{\pi}{3}-\alpha\right)-\left(\frac{\pi}{3}-\alpha\right)\right] \\
& \beta_{l, w}=12 \sin \left(\frac{\pi}{3}-\alpha\right) \\
& \beta_{i}=6\left(\frac{\pi}{3}-\alpha\right)
\end{aligned}
$$

Now, the first four equations are presented.

\section{Laplace-Young}

$$
p_{v}-p_{l}=\frac{\sigma}{r}
$$

And in differential format respect to $z$,

$$
\frac{\partial p_{v}}{\partial z}-\frac{\partial p_{l}}{\partial z}=-\frac{\sigma}{r^{2}} \frac{\partial r}{\partial z}
$$


Since the vapour pressure is independent of position, the first term equals to zero, therefore

$$
\frac{\partial p_{l}}{\partial z}=\frac{\sigma}{r^{2}} \frac{\partial r}{\partial z}
$$

\section{Liquid pressure drop}

$$
\frac{\partial p_{l}}{\partial z}=\frac{\partial p_{\text {fric }, l}}{\partial z}+\frac{\partial p_{\text {mom }, l}}{\partial z}
$$

where

$$
\frac{\partial p_{\text {fric, }, l}}{\partial z}=\frac{2 \mu v_{l}}{r_{h, l}^{2}}
$$

and

$$
\frac{\partial p_{\text {mom }, l}}{\partial z}=\frac{1}{A_{l}}\left(v_{l} \frac{\partial \dot{m}_{l}}{\partial z}+\dot{m}_{l} \frac{\partial v_{l}}{\partial z}+\frac{\partial \dot{m}_{l} \partial v_{l}}{\partial z^{2}}\right)
$$

Considering only the first order terms, the last term vanishes. Moreover $\frac{\partial \dot{m}_{l}}{\partial z}$ can be explained as

$$
\begin{aligned}
& \frac{\partial \dot{m}_{l}}{\partial z}=\frac{\partial}{\partial z}\left(\rho_{l} v_{l} A_{l}\right) \\
& \frac{\partial \dot{m}_{l}}{\partial z}=\rho_{l} A_{l} \frac{\partial v_{l}}{\partial z}+\rho_{l} v_{l} \frac{\partial A_{l}}{\partial z}
\end{aligned}
$$

\section{Conservation of momentum for liquid}

$$
\rho_{l} \frac{D v_{l}}{D t}=-\frac{\partial p_{l}}{\partial z}+\rho_{l} B_{z, l}+\left(F_{v i s, l}\right)_{z}
$$

For a one-dimensional analysis, it can be simplified as

$$
\rho_{l}\left(v_{l} \frac{\partial v_{l}}{\partial z}+\frac{\partial v_{l}}{\partial t}\right)+\frac{\partial p_{l}}{\partial z}=\rho_{l} g \sin \psi+\frac{\tau A_{l, w}}{V_{l}}
$$

where the volume of liquid is

$$
V_{l}=A_{l} d z
$$


and

$$
\tau=\frac{1}{2} \rho_{l} v_{l}^{2} f
$$

where

$$
f=\frac{K}{\operatorname{Re}_{l}}
$$

and

$$
\operatorname{Re}_{l}=\frac{\rho_{l} v_{l} D_{h, l}}{\mu}
$$

in which $D_{h, l}$ is the hydraulic diameter of all three liquid arteries, and can be explained as

$$
D_{h, l}=\frac{4 A_{l}}{6\left[2 r \sin \left(\frac{\pi}{3}-\alpha\right)\right]+3\left[2 r\left(\frac{\pi}{3}-\alpha\right)\right]}
$$

where $K=16$ for circular and 13.3 for triangular.

\section{Conservation of momentum for vapour$$
\rho_{v} \frac{D v_{v}}{D t}=-\frac{\partial p_{v}}{\partial z}+\rho_{v} B_{z, v}+\left(F_{v i s, v}\right)_{z}
$$

According to the assumptions

$$
\frac{\partial p_{v}}{\partial z}=0
$$

Thus, the conversation of momentum for the vapour phase can be written as

$$
\rho_{v}\left(v_{v} \frac{\partial v_{v}}{\partial z}+\frac{\partial v_{v}}{\partial t}\right)=\rho_{v} g \sin \psi+\frac{\tau A_{v, w}}{V_{v}}
$$

where

$$
\begin{aligned}
& V_{v}=A_{v} d z \\
& \tau=\frac{1}{2} \rho_{v} v_{v}^{2} f \\
& f=\frac{K}{\operatorname{Re}}
\end{aligned}
$$




$$
\operatorname{Re}_{v}=\frac{\rho_{v} v_{v} D_{h, v}}{\mu_{v}}
$$

In which

$$
D_{h, v}=\frac{4 A_{v}}{3\left[d-4 r \sin \left(\frac{\pi}{3}-\alpha\right)\right]+3\left[2 r\left(\frac{\pi}{3}-\alpha\right)\right]}
$$

According to [24] for the shape of the vapour flow $K=14.7$.

\section{Conservation of mass}

When $r, v_{l}, v_{v}$ and $p_{l}$ are determined, with the conservation of mass, the amount of phase change heat transfer can be achieved. Conservation of mass for a control volume of liquid requires (see Figure A-2)

$$
\dot{m}_{l}=\dot{m}_{l}+\frac{\partial \dot{m}_{l}}{\partial z} d z+\dot{m}_{p}+\frac{\partial m_{l}}{\partial t}
$$

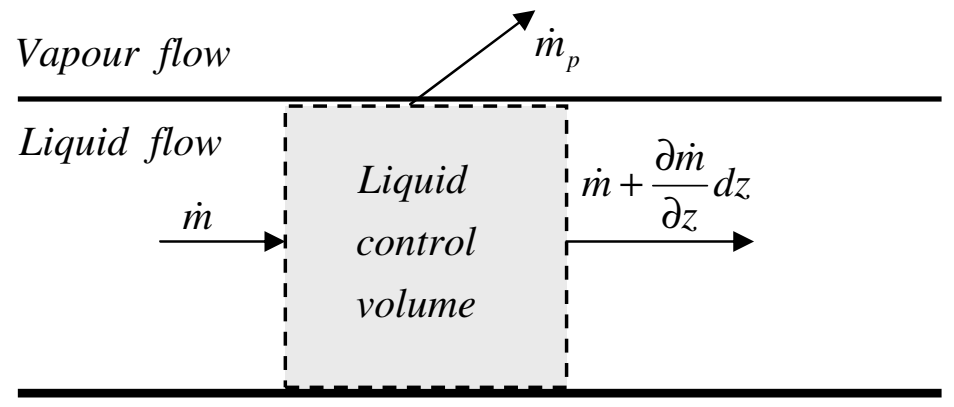

Figure A-2: Conservation of mass for a liquid control volume

It can be rewritten as

$$
\frac{\partial\left(\rho_{l} v_{l} A_{l}\right)}{\partial z} d z+\frac{\dot{Q}_{p} r d z}{\lambda}+\frac{\partial\left(\rho_{l} A_{l} d z\right)}{\partial t}=0
$$

Dividing by $d z$

$$
\frac{\partial\left(\rho_{l} v_{l} A_{l}\right)}{\partial z}+\frac{\dot{Q}_{p} r}{\lambda}+\frac{\partial\left(\rho_{l} A_{l}\right)}{\partial t}=0
$$

From this, knowing all other parameters $\dot{Q}_{p}$ can be determined. 


\section{Conservation of energy for liquid}

The conservation of energy for the liquid control volume shown in Figure A-3 can be written as

$$
\dot{E}_{\text {in }}+\dot{Q}_{\text {in }}-\dot{E}_{\text {out }}-\dot{E}_{p}=\frac{\partial U_{l}}{\partial t}
$$

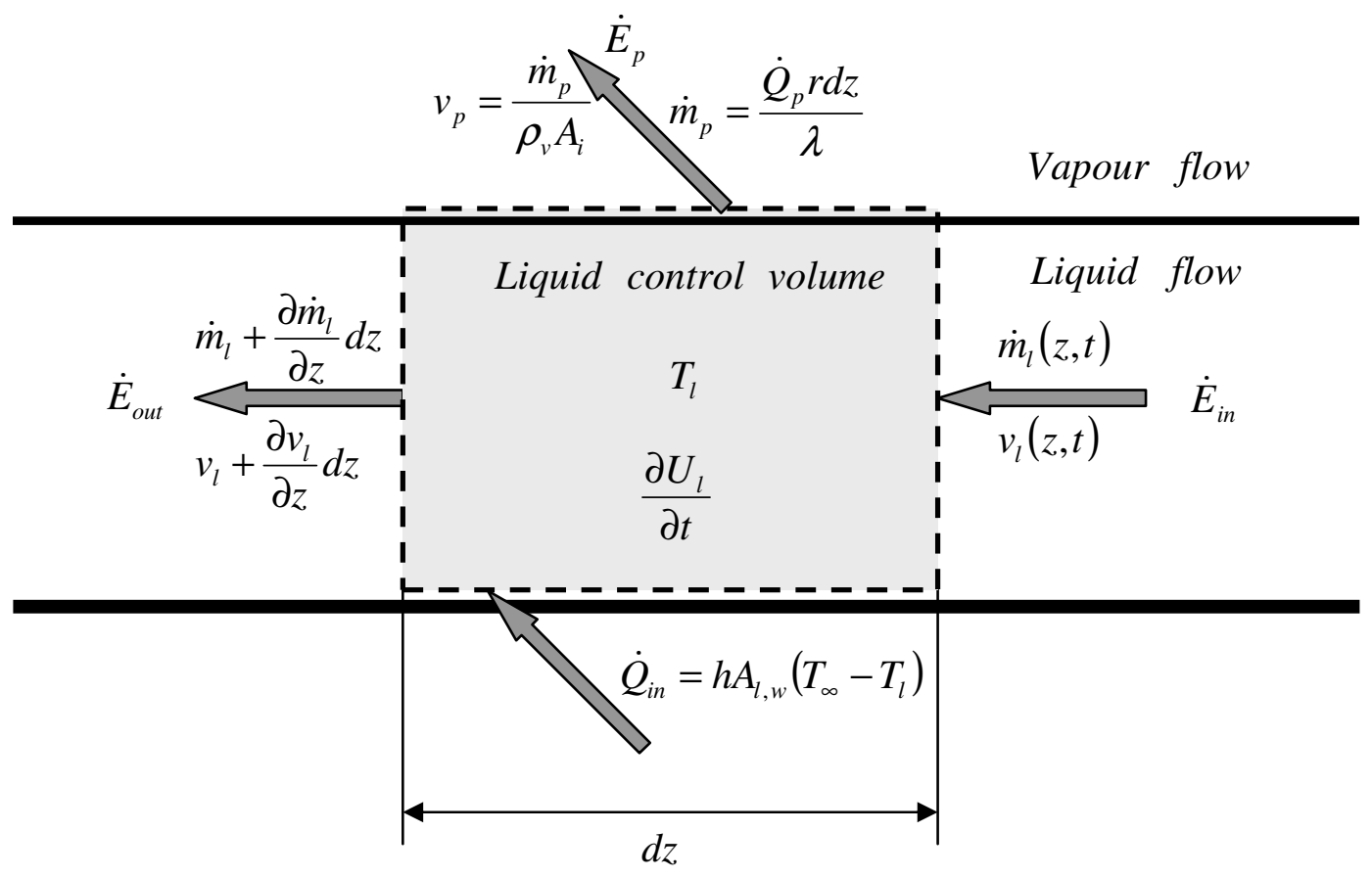

Figure A-3: Conservation of energy for a liquid control volume

Now, all the terms are substitute according to the following equations:

$$
\dot{E}_{i n}=K \dot{E}_{i n}+P \dot{E}_{i n}+\dot{H}_{i n}
$$

in which

$$
\begin{aligned}
& K \dot{E}_{i n}=\frac{1}{2} \dot{m}_{l} v_{l}^{2} \\
& P \dot{E}_{i n}=\dot{m}_{l} g z \sin \psi \\
& \dot{H}_{\text {in }}=\dot{m}_{l} h_{l}
\end{aligned}
$$


and

$$
\dot{E}_{\text {out }}=K \dot{E}_{\text {out }}+P \dot{E}_{\text {out }}+\dot{H}_{\text {out }}
$$

where

$$
\begin{aligned}
& K \dot{E}_{\text {out }}=\frac{1}{2}\left(\dot{m}_{l}+\frac{\partial \dot{m}_{l}}{\partial z} d z\right)\left(v_{l}+\frac{\partial v_{l}}{\partial z} d z\right)^{2} \\
& K \dot{E}_{\text {out }}=\frac{1}{2}\left(\dot{m}_{l}+\frac{\partial \dot{m}_{l}}{\partial z} d z\right)\left[v_{l}^{2}+\left(\frac{\partial v_{l}}{\partial z} d z\right)^{2}+2 v_{l} \frac{\partial v_{l}}{\partial z} d z\right] \\
& K \dot{E}_{\text {out }}=\frac{1}{2} \dot{m}_{l} v_{l}^{2}+\dot{m}_{l} v_{l} \frac{\partial v_{l}}{\partial z} d z+\frac{1}{2} v_{l}^{2} \frac{\partial \dot{m}_{l}}{\partial z} d z \\
& P \dot{E}_{\text {out }}=\left(\dot{m}_{l}+\frac{\partial \dot{m}_{l}}{\partial z} d z\right) g(z+d z) \sin \psi \\
& P \dot{E}_{\text {out }}=\dot{m}_{l} g z \sin \psi+\dot{m}_{l} g \sin \psi d z+\frac{\partial \dot{m}_{l}}{\partial z} g z \sin \psi d z \\
& \dot{H}_{\text {out }}=\left(\dot{m}_{l}+\frac{\partial \dot{m}_{l}}{\partial z} d z\right) h_{l}
\end{aligned}
$$

Furthermore

$$
\dot{Q}_{i n}=h A_{l, w}\left(T_{\infty}-T_{l}\right)
$$

and

$$
\begin{aligned}
& \dot{E}_{p}=K \dot{E}_{p}+P \dot{E}_{p}+\dot{H}_{p} \\
& K \dot{E}_{p}=\frac{1}{2} \dot{m}_{p} v_{p}^{2} \\
& K \dot{E}_{p}=\frac{1}{2}\left(\frac{\dot{Q}_{p} r d z}{\lambda}\right)\left(\frac{\frac{\dot{Q}_{p} r d z}{\lambda}}{\rho_{v} A_{i}}\right)^{2} \\
& K \dot{E}_{p}=\frac{\dot{Q}_{p}^{3} r}{2 \lambda^{3} \rho_{v}^{2} \beta_{i}^{2}} d z \\
& P \dot{E}_{p}=\dot{m}_{p} g\left(z+\frac{d z}{2}\right) \sin \psi
\end{aligned}
$$




$$
\begin{aligned}
& P \dot{E}_{p}=\frac{\dot{Q}_{p} r d z}{\lambda} g\left(z+\frac{d z}{2}\right) \sin \psi \\
& P \dot{E}_{p}=\frac{\dot{Q}_{p} r}{\lambda} g z \sin \psi d z \\
& \dot{H}_{p}=\dot{m}_{p} h_{v} \\
& \dot{H}_{p}=\frac{\dot{Q}_{p} r}{\lambda} h_{v} d z
\end{aligned}
$$

And Finally,

$$
\begin{aligned}
& \frac{d U_{l}}{d t}=\frac{d}{d t}\left(m_{l} u_{l}\right) \\
& \frac{d U_{l}}{d t}=\dot{m}_{l} u_{l}-\left(\dot{m}_{l}+\frac{\partial \dot{m}_{l}}{\partial z} d z\right) u_{l}-\dot{m}_{p} u_{v}+m_{l} C_{P, l} \frac{\partial T_{l}}{\partial t} \\
& \frac{d U_{l}}{d t}=-\frac{\partial \dot{m}_{l}}{\partial z} u_{l} d z-\frac{\dot{Q}_{p} r}{\lambda} u_{v} d z+\rho_{l} \beta_{l} r^{2} C_{P, l} \frac{\partial T_{l}}{\partial t} d z
\end{aligned}
$$

\section{Conservation of energy for vapour}

Similar to the liquid phase, the conservation of energy can be written for the vapour phase (see Figure A-4).

$$
\dot{E}_{\text {in }}+\dot{Q}_{\text {in }}-\dot{E}_{\text {out }}+\dot{E}_{p}=\frac{\partial U_{v}}{\partial t}
$$

where all the terms can be substitute as follows

$$
\dot{E}_{\text {in }}=K \dot{E}_{\text {in }}+P \dot{E}_{\text {in }}+\dot{H}_{\text {in }}
$$

in which

$$
\begin{aligned}
& K \dot{E}_{i n}=\frac{1}{2} \dot{m}_{v} v_{v}^{2} \\
& P \dot{E}_{i n}=\dot{m}_{v} g z \sin \psi \\
& \dot{H}_{i n}=\dot{m}_{v} h_{v}
\end{aligned}
$$

and

$$
\dot{E}_{\text {out }}=K \dot{E}_{\text {out }}+P \dot{E}_{\text {out }}+\dot{H}_{\text {out }}
$$




$$
\begin{aligned}
& K \dot{E}_{\text {out }}=\frac{1}{2} \dot{m}_{v} v_{v}^{2}+\dot{m}_{v} v_{v} \frac{\partial v_{v}}{\partial z} d z+\frac{1}{2} v_{v}^{2} \frac{\partial \dot{m}_{v}}{\partial z} d z \\
& P \dot{E}_{\text {out }}=\dot{m}_{v} g z \sin \psi+\dot{m}_{v} g \sin \psi d z+\frac{\partial \dot{m}_{v}}{\partial z} g z \sin \psi d z \\
& \dot{H}_{\text {out }}=\left(\dot{m}_{v}+\frac{\partial \dot{m}_{v}}{\partial z} d z\right) h_{v}
\end{aligned}
$$

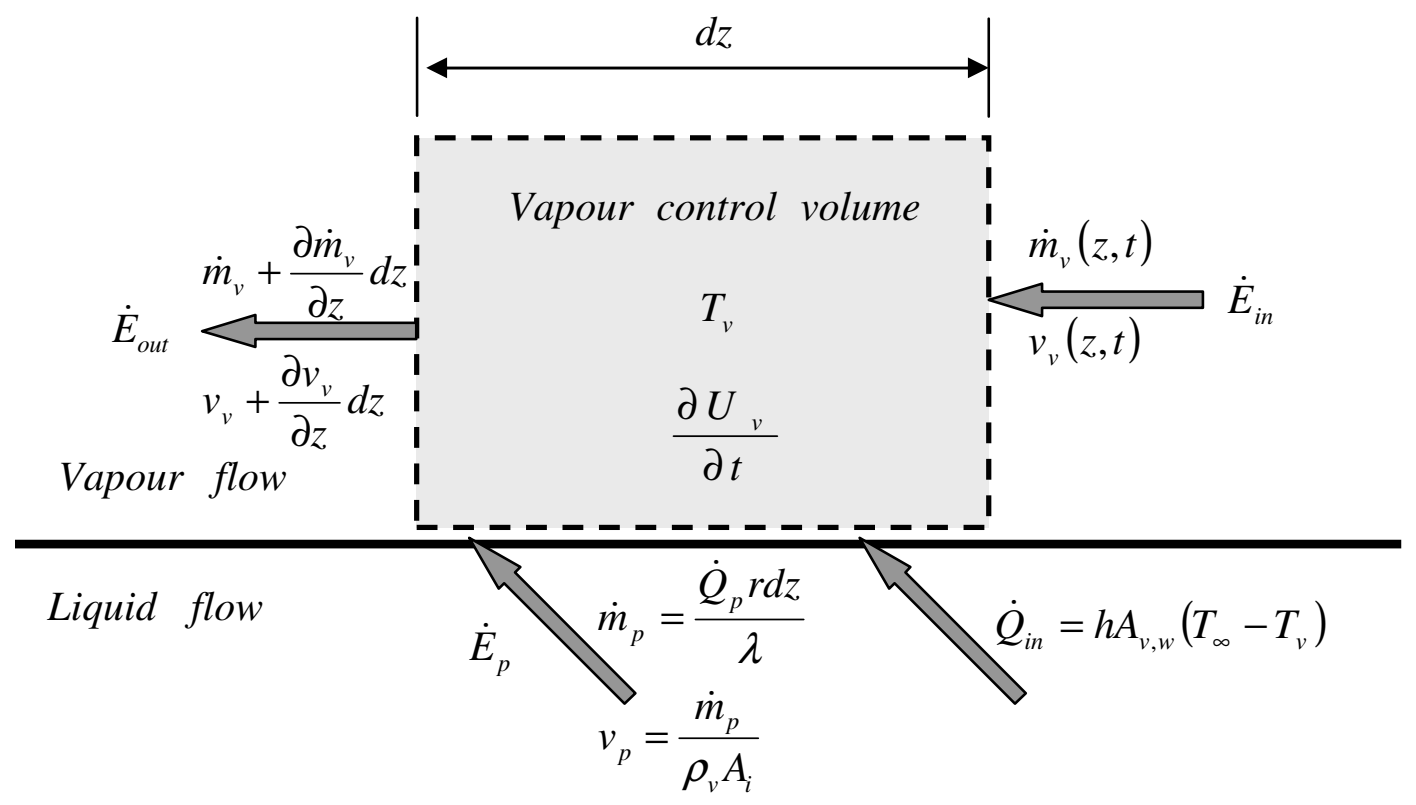

Figure A-4: Conservation of energy for a vapour control volume

Furthermore

$$
\dot{Q}_{i n}=h A_{v, w}\left(T_{\infty}-T_{v}\right)
$$

and

$$
\begin{aligned}
& \dot{E}_{p}=K \dot{E}_{p}+P \dot{E}_{p}+\dot{H}_{p} \\
& K \dot{E}_{p}=\frac{\dot{Q}_{p}^{3} r}{2 \lambda^{3} \rho_{v}^{2} \beta_{i}^{2}} d z \\
& P \dot{E}_{p}=\frac{\dot{Q}_{p} r}{\lambda} g z \sin \psi d z
\end{aligned}
$$




$$
\dot{H}_{p}=\frac{\dot{Q}_{p} r}{\lambda} h_{v} d z
$$

And finally,

$$
\begin{aligned}
& \frac{d U_{v}}{d t}=\frac{d}{d t}\left(m_{v} u_{v}\right) \\
& \frac{d U_{v}}{d t}=\dot{m}_{v} u_{v}-\left(\dot{m}_{v}+\frac{\partial \dot{m}_{v}}{\partial z} d z\right) u_{v}+\dot{m}_{p} u_{v}+m_{v} C_{P, v} \frac{\partial T_{v}}{\partial t} \\
& \frac{d U_{v}}{d t}=-\frac{\partial \dot{m}_{v}}{\partial z} u_{v} d z+\frac{\dot{Q}_{p} r}{\lambda} u_{v} d z+\rho_{v}\left(\frac{\sqrt{3}}{4} d^{2}-\beta_{l} r^{2}\right) C_{P, v} \frac{\partial T_{v}}{\partial t} d z
\end{aligned}
$$

